\title{
Cobalt-catalyzed $E-(\beta)$-selective hydrogermylation of terminal alkynes
}

\author{
Maxim R. Radzhabov and Neal P. Mankad* \\ Department of Chemistry, University of Illinois at Chicago \\ 845 W. Taylor St., Chicago, IL 60607, USA. \\ *email: npm@uic.edu
}

\section{Supporting Information}

\section{Table of contents}

1. General information $\underline{\mathrm{S}-2}$

2. General procedure for optimization $\underline{\text { S-3 }}$

3. General procedure for hydrogermylation S-8

4. Upscaled procedure for hydrogermylation of 1-decyne $\underline{\text { S-18 }}$

5. Mechanistic studies $\underline{\text { S-19 }}$

6. ${ }^{1} \mathrm{H},{ }^{13} \mathrm{C}\left\{{ }^{1} \mathrm{H}\right\}$ NMR spectra $\underline{\text { S-20 }}$

7. References $\underline{\text { S-44 }}$ 


\section{General information}

General Procedures. Reactions requiring anhydrous conditions were conducted in a $\mathrm{N}_{2}-$ filled glovebox or using standard Schlenk line techniques. Reactions at low temperature were conducted using a Flow Through Cooler made by PolyScience. Purification of compounds was achieved by flash column chromatography using SiliCycle SiliaFlash F60 Silica Gel (230-400 mesh). *Silica Gel with lower mesh values may lead to worse separation. Organic solutions were concentrated under reduced pressure using a rotary evaporator or by standard Schlenk line techniques.

Materials. Deuterated solvents were purchased from Cambridge Isotope Laboratories, Inc and used as is. Common commercial reagents were purchased from Sigma-Aldrich, Fisher Scientific or VWR International Co and used without further purification (unless otherwise noted). Solvents were dried using a Glass Contour Solvent System built by Pure Process Technology, LLC. Bu3 $\mathrm{GeH}$ was purchased from Fisher Scientific or Gelest and used without further purification. IMesCuCl, ${ }^{1} \mathrm{NaMoCp}(\mathrm{CO})_{3},{ }^{2} \mathrm{NaWCp}(\mathrm{CO})_{3},{ }^{3}$ $\mathrm{NaCrCp}(\mathrm{CO})_{3},{ }^{2} \mathrm{NaCo}(\mathrm{CO})_{4},{ }^{2} \mathrm{NaMn}(\mathrm{CO}) 5,{ }^{2}$ and $\mathrm{KFp}^{4}$ were prepared according to literature procedures.

Instrumentation. Nuclear magnetic resonance (NMR) spectra were recorded on Bruker $\mathrm{AV}(400 \mathrm{MHz})$ at $298 \mathrm{~K}$. Chemical shifts $(\delta)$ are reported in ppm with the residual solvent signal as internal standard (chloroform at 7.26 and $77.00 \mathrm{ppm}$ for ${ }^{1} \mathrm{H}$ and ${ }^{13} \mathrm{C}\left\{{ }^{1} \mathrm{H}\right\} \mathrm{NMR}$ spectroscopy, respectively). Abbreviations for signal coupling are as follows: s, singlet; $d$, doublet; t, triplet; q, quartet; quin., quintet; m, multiplet; br., broad. Coupling constants were taken from the spectra directly and are uncorrected. ${ }^{1} \mathrm{H}$ and ${ }^{13} \mathrm{C}\left\{{ }^{1} \mathrm{H}\right\}$ NMR provided are taken directly using material for which the yield is quoted, without further purification, and are representative of purity. Electrospray ionization (ES) high resolution (HR) mass spectra were recorded on a Waters Micromass Q-TOF Ultima mass spectrometer at the University of Illinois at Urbana-Champaign (UIUC). Electron impact (EI) and Chemical Ionization $(\mathrm{Cl}) \mathrm{HR}$ mass spectra were recorded using a Micromass 70-VSE TOF mass spectrometer at UIUC. 


\section{Preparation of some starting alkynes.}

Commercially available chemicals were purchased from the vendors listed below and used without further purification.

1-decyne, cyclohexylacetylene, 2,2-dimethyl-3-butyne, methyl propiolate, 1ethynyl-3,5-bis(trifluoromethyl)benzene, 2-ethynyl-6-methoxynaphthalene, Mestranol and ethynyltrimethylsilane were purchased from Sigma-Aldrich. 5-phenyl-1-pentyne, methyl hex-5-ynoate, cyclopropylacetylene, 6-chloro-1-hexyne, 5-hexynenitrile, phenylacetylene, 4-fluorophenylacetylene, 4-methoxyphenylacetylene and undec-10-yn1-ol were purchased from Alfa Aesar. 5-decyne was purchased from VWR. $\mathrm{Bu}_{3} \mathrm{GeH}$ was purchased from Sigma-Aldrich, Alfa Aesar and Gelest. $\mathrm{Ph}_{3} \mathrm{GeH}$ was purchased from Gelest.

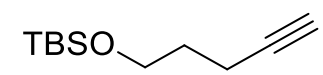

1-(tert-Butyldimethylsilyloxy)-4-pentyne was prepared according to the known literature and has been previously characterized. ${ }^{5}$

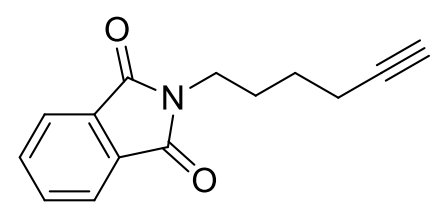

$\mathrm{N}$-(Hex-5-ynyl)-phthalimide was prepared according to the known literature and has been previously characterized. ${ }^{6}$

\section{General procedure for optimization}

In a glovebox, a catalyst $(0.02 \mathrm{mmol})(+$ co-catalyst $(0.02 \mathrm{mmol})$ and $/$ or additives if applicable) and solvent $(2.0 \mathrm{~mL})$ were added to a $20-\mathrm{mL}$ vial with a $1.5 \mathrm{~cm}$ stir bar. The mixture was stirred at room temperature for 10-15 min before 1-decyne (36.0 $\mu \mathrm{L}, 0.2$ $\mathrm{mmol}, 1.0$ eq or $40.0 \mu \mathrm{L}, 0.22 \mathrm{mmol}, 1.1 \mathrm{eq})$ and $\mathrm{Bu}_{3} \mathrm{GeH}(62.0 \mu \mathrm{L}, 0.24 \mathrm{mmol}, 1.2 \mathrm{eq}$ or $52.0 \mu \mathrm{L}, 0.2 \mathrm{mmol}, 1.0 \mathrm{eq}$ ) were added via Hamilton glass microsyringes to the reaction mixture. $\left({ }^{*}\right)$ After addition was complete, the reaction was put into a pre-heated hot plate and stirred for $15 \mathrm{~h} .\left(^{*}\right)$ Then the vial was cooled down to room temperature, taken out of the glovebox, and the solvent was removed under reduced pressure via Schlenk line. The residue was filtered twice through a pad of silica gel (a pipette with about $4 \mathrm{~cm}$ silica gel) using diethyl ether $\left(\mathrm{Et}_{2} \mathrm{O}\right)$ as the eluent. The filtrate was concentrated under reduced 
pressure. The residue was dissolved in $\mathrm{CDCl}_{3}$, and $\mathrm{CH}_{2} \mathrm{Br}_{2}(0.1 \mathrm{mmol}, 7 \mu \mathrm{L})$ was added as internal standard for ${ }^{1} \mathrm{H}$ NMR analysis.

$\left(^{*}\right)$ The procedure for low-temperature reactions is somewhat different and is as follows. The solution of catalytic system was stirred at room temperature for $20 \mathrm{~min}$ in a chosen solvent before 1-decyne $(36.0 \mu \mathrm{L}, 0.2 \mathrm{mmol}, 1.0 \mathrm{eq})$ was added at room temperature via a $50.0 \mu \mathrm{L}$ Hamilton glass microsyringe. Then the vial was cooled down in the glovebox using a dry ice/acetone bath, and $\mathrm{Bu}_{3} \mathrm{GeH}(62.0 \mu \mathrm{L}, 0.24 \mathrm{mmol}, 1.2 \mathrm{eq})$ was added via $100.0 \mu \mathrm{L}$ Hamilton glass microsyringe to the reaction mixture. The vial then was taken out of the glovebox, cooled down to $-10{ }^{\circ} \mathrm{C}$ using a low temperature cooler, and left stirring for $15 \mathrm{~h}$. Upon reaction completion the vial was unsealed, and the solvent was removed under reduced pressure via Schlenk line. The residue was filtered twice through a pad of silica gel (a pipette with about $4 \mathrm{~cm}$ silica gel) using diethyl ether $\left(\mathrm{Et}_{2} \mathrm{O}\right)$ as the eluent. The filtrate was concentrated under reduced pressure. The residue was dissolved in $\mathrm{CDCl}_{3}$, and $\mathrm{CH}_{2} \mathrm{Br}_{2}(0.1 \mathrm{mmol}, 7 \mu \mathrm{L})$ was added as internal standard for ${ }^{1} \mathrm{H}$ NMR analysis. 


\subsection{Optimization tables for the synthesis of E-( $\beta$ )-vinylgermanes}

Table S1. Initial catalyst and temperature investigation

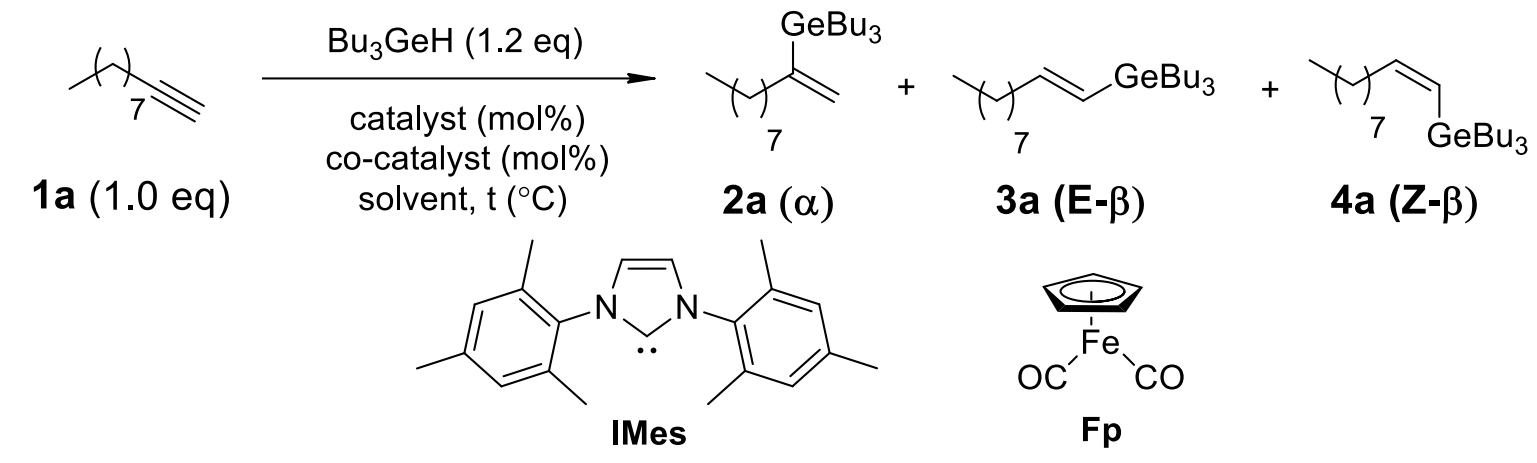

\begin{tabular}{cccccccc}
\hline Entry & Catalytic system & $\mathrm{T}\left({ }^{\circ} \mathrm{C}\right)$ & $\mathrm{Time}$ & Solvent & 2a (\%) & 3a (\%) & 4a (\%) \\
\hline 1 & $\mathrm{IMesCuCl}(10 \%), \mathrm{NaMn}(\mathrm{CO})_{5}(10 \%)$ & 60 & $4 \mathrm{~h}$ & $\mathrm{DCE}$ & 0 & 0 & 0 \\
2 & $\mathrm{IMesCuCl}(12 \%), \mathrm{KFp}(8 \%)$ & -10 & $12 \mathrm{~h}$ & toluene & 1 & 2 & 24 \\
3 & $\mathrm{IMesCuCl}(10 \%), \mathrm{NaMn}(\mathrm{CO})_{5}(10 \%)$ & 110 & $4 \mathrm{~h}$ & toluene & 0 & 0 & traces \\
4 & $\mathrm{IMesCuCl}(10 \%), \mathrm{NaCo}(\mathrm{CO})_{4}(10 \%)$ & 110 & $4 \mathrm{~h}$ & toluene & 23 & 22 & 1 \\
5 & $\mathrm{IMesCuCl}(10 \%), \mathrm{NaCrCp}(\mathrm{CO})_{3}(10 \%)$ & 110 & $4 \mathrm{~h}$ & toluene & 0 & 0 & 0 \\
6 & $\mathrm{IMesCuCl}(10 \%), \mathrm{NaWCp}(\mathrm{CO})_{3}(10 \%)$ & 110 & $4 \mathrm{~h}$ & toluene & 0 & 0 & 0 \\
7 & $\mathrm{IMesCuCl}(10 \%), \mathrm{NaMoCp}(\mathrm{CO})_{3}(10 \%)$ & 110 & $4 \mathrm{~h}$ & toluene & 0 & 0 & 0 \\
8 & $\mathrm{NaCo}(\mathrm{CO})_{4}(10 \%)$ & 110 & $4 \mathrm{~h}$ & toluene & 43 & 37 & 0 \\
9 & $\mathrm{NaCo}(\mathrm{CO})_{4}(10 \%)$ & 100 & $15 \mathrm{~h}$ & toluene & 56 & 44 & 0 \\
10 & $\mathrm{NaCo}(\mathrm{CO})_{4}(10 \%)$ & 60 & $15 \mathrm{~h}$ & toluene & 18 & 18 & 8 \\
11 & $\mathrm{NaCo}(\mathrm{CO})_{4}(10 \%)$ & $\mathrm{RT}$ & $15 \mathrm{~h}$ & toluene & traces & 4 & 36 \\
12 & $\mathrm{NaCo}(\mathrm{CO})_{4}(10 \%)$ & -10 & $15 \mathrm{~h}$ & toluene & 0 & 5 & 40 \\
\hline
\end{tabular}


Table S2. Additives influence investigation

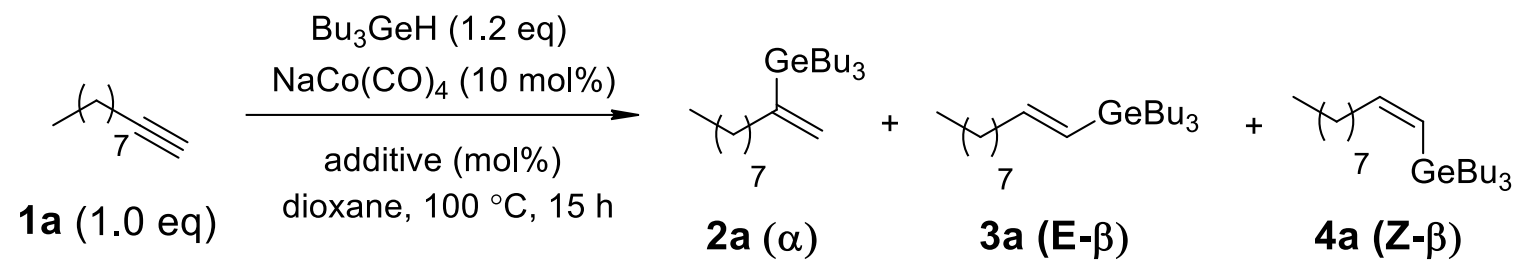

\begin{tabular}{cccccc}
\hline Entry & Additive (mol\%) & $\mathrm{Bu}_{3} \mathrm{GeH}$ (eq.) & $\mathbf{2 a}(\%)$ & $\mathbf{3 a}(\%)$ & 4a (\%) \\
\hline 1 & none & 1.2 & 29 & 20 & 0 \\
2 & $\mathrm{Bu}_{4} \mathrm{NBr}(10 \%)$ & 1.0 & 15 & 10 & 1 \\
3 & $\mathrm{Bu}_{4} \mathrm{NPF}_{6}(10 \%)$ & 1.0 & 16 & 12 & 2 \\
4 & $\mathrm{Bu}_{4} \mathrm{NBF}_{4}(10 \%)$ & 1.0 & 40 & 27 & 2 \\
5 & $15-$ crown-5 (30\%) $^{-10}$ & 1.0 & 19 & 13 & 4 \\
\hline
\end{tabular}

Table S3. Further catalyst investigation

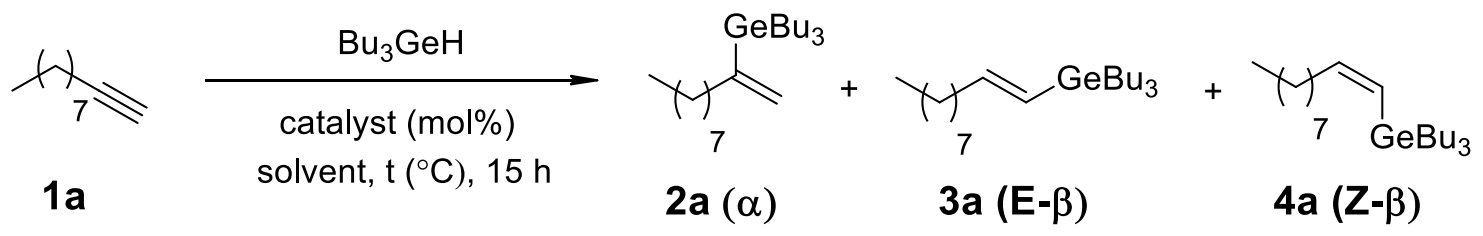

\begin{tabular}{ccccccccc}
\hline Entry & Catalyst & $\mathrm{T}\left({ }^{\circ} \mathrm{C}\right)$ & 1a (eq.) & $\mathrm{Bu}_{3} \mathrm{GeH}($ eq. $)$ & Solvent & 2a (\%) & 3a (\%) & 4a (\%) \\
\hline 1 & $\left.\mathrm{~K}_{2}\left[\mathrm{Fe}(\mathrm{CO})_{4}\right)\right](10 \%)$ & 100 & 1.0 & 1.2 & toluene & 0 & 0 & 0 \\
2 & $\mathrm{KFp}(10 \%)$ & 100 & 1.0 & 1.2 & toluene & 0 & 0 & 4 \\
3 & $\mathrm{Na}\left[\mathrm{Co}(\mathrm{CO})_{3} \mathrm{PPh}_{3}\right](10 \%)$ & 100 & 1.0 & 1.2 & toluene & 12 & 27 & 0 \\
4 & $\mathrm{Fe}(\mathrm{CO})_{5}(10 \%)$ & 100 & 1.1 & 1.0 & toluene & 6 & 7 & 2 \\
5 & $\mathrm{Ni}(\mathrm{COd})_{2}(10 \%)$ & 100 & 1.1 & 1.0 & toluene & 0 & 0 & 0 \\
6 & $\mathrm{~K}_{3}\left[\mathrm{Co}(\mathrm{CN})_{6}\right](10 \%)$ & 100 & 1.1 & 1.0 & toluene & 0 & 0 & 1 \\
7 & $\mathrm{Mn}_{2}(\mathrm{CO})_{10}(10 \%)$ & $\mathrm{RT}, \mathrm{hv}$ & 1.0 & 1.2 & $\mathrm{DCM}$ & traces & 2 & 20 \\
8 & $\mathrm{Co}_{2}(\mathrm{CO})_{8}(10 \%)$ & $\mathrm{RT}, \mathrm{hv}$ & 1.0 & 1.2 & $\mathrm{DCM}$ & 16 & 24 & 16 \\
9 & $\mathrm{Co}_{2}(\mathrm{CO})_{8}(10 \%)$ & 85 & 1.0 & 1.2 & $\mathrm{DCE}$ & 10 & 55 & 6 \\
10 & $\left.\mathrm{CPCo}_{2} \mathrm{CO}\right)_{2}(10 \%)$ & 85 & 1.0 & 1.2 & $\mathrm{DCE}$ & 2 & 5 & 5 \\
\hline
\end{tabular}


Table S4. Solvent screening

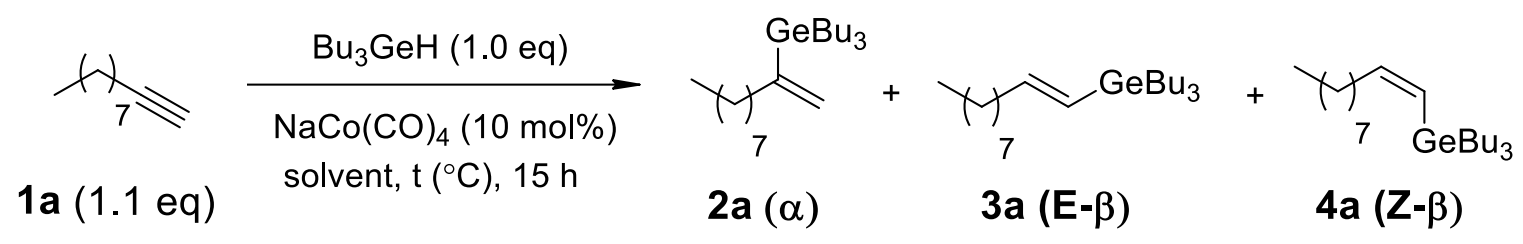

\begin{tabular}{cccccc}
\hline Entry & $\mathrm{T}\left({ }^{\circ} \mathrm{C}\right)$ & Solvent & 2a (\%) & 3a (\%) & 4a (\%) \\
\hline 1 & 100 & toluene & 29 & 44 & 1 \\
2 & 100 & DMF & 7 & 4 & 0 \\
3 & 85 & DCE & 19 & 81 & 0 \\
4 & 60 & THF & 6 & 3 & 5 \\
\hline
\end{tabular}

Table S5. Reaction conditions optimization

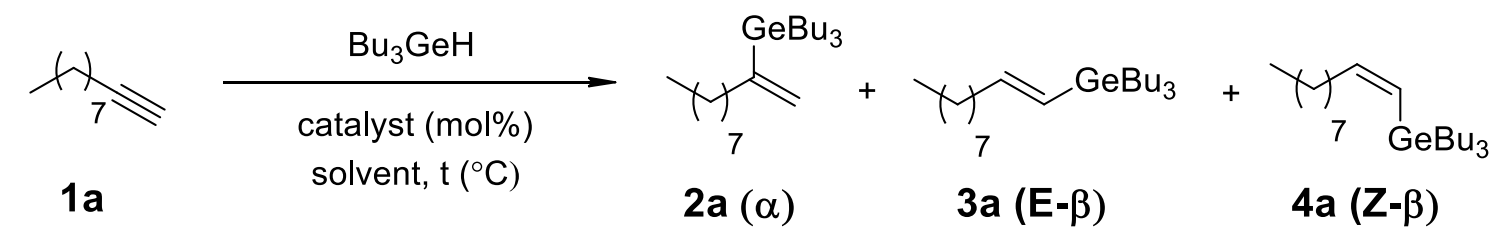

\begin{tabular}{ccccccccc}
\hline entry & Catalyst & $\mathrm{T}\left({ }^{\circ} \mathrm{C}\right)$ & 1a (eq.) & $\mathrm{Bu}_{3} \mathrm{GeH}$ (eq.) & time & 2a (\%) & 3a (\%) & 4a (\%) \\
\hline 1 & $\mathrm{Co}_{2}(\mathrm{CO})_{8}(10 \%)$ & 85 & 1.0 & 1.2 & $15 \mathrm{~h}$ & 10 & 55 & 6 \\
2 & $\mathrm{Co}_{2}(\mathrm{CO})_{8}(10 \%)$ & 85 & 1.0 & 1.0 & $15 \mathrm{~h}$ & 8 & 80 & traces \\
3 & $\mathrm{Co}_{2}(\mathrm{CO})_{8}(10 \%)$ & 85 & 1.1 & 1.0 & $15 \mathrm{~h}$ & 8 & 90 & 0 \\
4 & $\mathrm{Co}_{2}(\mathrm{CO})_{8}(10 \%)$ & 85 & 1.2 & 1.0 & $15 \mathrm{~h}$ & 8 & 88 & 0 \\
5 & $\mathrm{Co}_{2}(\mathrm{CO})_{8}(10 \%)$ & 85 & 1.0 & 2.5 & $15 \mathrm{~h}$ & 8 & 54 & 3 \\
6 & {$\left[\mathrm{Co}(\mathrm{CO})_{3} \mathrm{PEt}_{3}\right]_{2}(10 \%)$} & 85 & 1.1 & 1.0 & $15 \mathrm{~h}$ & 11 & 65 & 2 \\
7 & $\mathrm{Co}_{\left(\mathrm{PMe}_{3}\right)_{4}(10 \%)}$ & 85 & 1.1 & 1.0 & $15 \mathrm{~h}$ & 0 & 0 & 0 \\
8 & $\mathrm{Co}_{2}(\mathrm{CO})_{8}(10 \%)$ & 70 & 1.1 & 1.0 & $15 \mathrm{~h}$ & 9 & 91 & 0 \\
9 & $\mathrm{Co}_{2}(\mathrm{CO})_{8}(10 \%)$ & 60 & 1.1 & 1.0 & $15 \mathrm{~h}$ & 9 & 91 & 0 \\
10 & $\mathrm{Co}_{2}(\mathrm{CO})_{8}(10 \%)$ & 40 & 1.1 & 1.0 & $15 \mathrm{~h}$ & 16 & 73 & 0 \\
11 & $\mathrm{Co}_{2}(\mathrm{CO})_{8}(10 \%)$ & $\mathrm{RT}$ & 1.1 & 1.0 & $15 \mathrm{~h}$ & 10 & 14 & 2 \\
12 & $\mathrm{Co}_{2}(\mathrm{CO})_{8}(5 \%)$ & 60 & 1.1 & 1.0 & $15 \mathrm{~h}$ & 12 & 85 & 0 \\
13 & $\mathrm{Co}_{2}(\mathrm{CO})_{8}(15 \%)$ & 60 & 1.1 & 1.0 & $15 \mathrm{~h}$ & 9 & 87 & 0 \\
14 & $\mathrm{Co}_{2}(\mathrm{CO})_{8}(10 \%)$ & 60 & 1.1 & 1.0 & $4 \mathrm{~h}$ & 9 & 86 & 0 \\
\hline
\end{tabular}




\section{General procedure for hydrogermylation of alkynes using $\mathrm{Co}_{2}(\mathrm{CO})_{8}$ as a catalyst}

In a glovebox, cobalt catalyst $\mathrm{Co}_{2}(\mathrm{CO})_{8}(6.9 \mathrm{mg}, 0.02 \mathrm{mmol}, 0.1 \mathrm{eq})$ was dissolved in $2.0 \mathrm{~mL}$ of DCE (1,2-dichloroethane) in a $20-\mathrm{mL}$ vial with a $0.5 \mathrm{~cm}$ stir bar. The mixture was stirred at room temperature for 10-15 min before the alkyne $(0.22 \mathrm{mmol}, 1.1 \mathrm{eq})$ and $\mathrm{Bu}_{3} \mathrm{GeH}(52.0 \mu \mathrm{L}, 0.02 \mathrm{mmol}, 1.0 \mathrm{eq})$ were added via Hamilton glass microsyringes (if applicable) to the reaction mixture. $\left({ }^{*}\right)$ After addition was complete, the reaction was put into a hot plate (that had been pre-heated to $60^{\circ} \mathrm{C}$ ) and stirred for $4 \mathrm{~h}$. Then the vial was taken out of the glovebox, and the solvent was removed under reduced pressure via Schlenk line. The residue was filtered twice through a pad of silica gel (a pipette with about $4 \mathrm{~cm}$ silica gel) using diethyl ether $\left(\mathrm{Et}_{2} \mathrm{O}\right)$ as the eluent. The filtrate was concentrated under reduced pressure. The residue was dissolved in $\mathrm{CDCl}_{3}$, and $\mathrm{CH}_{2} \mathrm{Br}_{2}$ $(0.1 \mathrm{mmol}, 7 \mu \mathrm{L})$ was added as internal standard for ${ }^{1} \mathrm{H}$ NMR analysis.

Apparently some red-coloring Co species (presumably $\mathrm{Co}_{2}(\mathrm{CO})_{8}$ ) remains in the reaction mixture after filtration; however, it can be eliminated by letting it oxidize for a day or two and then filtering the reaction mixture again. Another way to get rid of it would be to heat the reaction mixture up to $85^{\circ} \mathrm{C}$, because the Co species decomposes, and the product does not.

$\left({ }^{*}\right)$ If the alkyne was solid, then it was dissolved in $1.0 \mathrm{~mL}$ of DCE first and then added to the solution of $\mathrm{Co}_{2}(\mathrm{CO})_{8}$ in $1.0 \mathrm{~mL}$ of $\mathrm{DCE}$.

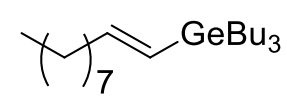

(E)-tributyl(dec-1-en-1-yl)germane (3a). Prepared according to the general procedure using 1-decyne ( $40.0 \mu \mathrm{L}, 0.22 \mathrm{mmol}, 1.1 \mathrm{eq})$. The crude material was purified by flash silica gel chromatography using $\mathrm{Et}_{2} \mathrm{O}$ as the eluent to afford a mixture of $\beta$-vinylgermane 3a and $\alpha$-vinylgermane $2 \mathbf{a}$ as dark brown oil $(75.4 \mathrm{mg}, 98 \%$ isolated yield). The ratio of $\beta / \alpha$ was determined to be $9.5: 1$ by the ${ }^{1} \mathrm{H}$ NMR analysis of the isolated mixture.

${ }^{1} \mathrm{H}$ NMR $\left(400 \mathrm{MHz}, \mathrm{CDCl}_{3}\right) \delta 5.90(\mathrm{dt}, J=18.3,6.3 \mathrm{~Hz}, 1 \mathrm{H}), 5.68(\mathrm{~d}, J=18.3 \mathrm{~Hz}, 1 \mathrm{H})$, 2.11 (dt, $J=6.3,6.4 \mathrm{~Hz}, 2 \mathrm{H}), 1.40-1.30(\mathrm{~m}, 15 \mathrm{H}$, merged), $1.30-1.25(\mathrm{~m}, 9 \mathrm{H}$, merged), 0.89 (t, $J=7.0 \mathrm{~Hz}, 12 \mathrm{H}), 0.81-0.69(\mathrm{~m}, 6 \mathrm{H})$.

${ }^{13} \mathrm{C}\left\{{ }^{1} \mathrm{H}\right\}$ NMR $\left(101 \mathrm{MHz}, \mathrm{CDCl}_{3}\right) \delta 146.3,127.2,36.8,31.9,29.5,29.3,29.1,28.9,27.4$, 26.5, 22.7, 14.1, 13.7, 12.9 .

HRMS (El+) m/z: [M]+ calcd for $\mathrm{C}_{22} \mathrm{H}_{46} \mathrm{Ge:}$ 384.2811; found: 384.2824 . 


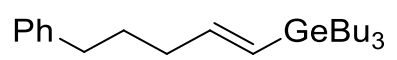

(E)-tributyl(5-phenylpent-1-en-1-yl)germane (3b). Prepared according to the general procedure using 5-phenyl-1-pentyne $(31.7 \mathrm{mg}, 0.22 \mathrm{mmol}, 1.1 \mathrm{eq})$. The crude material was purified by flash silica gel chromatography using $\mathrm{Et}_{2} \mathrm{O}$ as the eluent to afford a mixture of $\beta$-vinylgermane $\mathbf{3 b}$ and $\alpha$-vinylgermane $\mathbf{2 b}$ as red-brown oil $(72.1 \mathrm{mg}, 93 \%$ isolated yield). The ratio of $\beta / \alpha$ was determined to be $8.4: 1$ by the ${ }^{1} \mathrm{H}$ NMR analysis of the isolated mixture.

${ }^{1} \mathrm{H}$ NMR $\left(400 \mathrm{MHz}, \mathrm{CDCl}_{3}\right) \delta 7.33-7.27(\mathrm{~m}, 2 \mathrm{H}), 7.23-7.17(\mathrm{~m}, 3 \mathrm{H}), 5.95(\mathrm{dt}, J=18.3$, $6.2 \mathrm{~Hz}, 1 \mathrm{H}), 5.76(\mathrm{~d}, J=18.3 \mathrm{~Hz}, 1 \mathrm{H}), 2.64(\mathrm{t}, J=7.7 \mathrm{~Hz}, 2 \mathrm{H}), 2.19(\mathrm{dt}, J=6.9,6.2 \mathrm{~Hz}, 2 \mathrm{H}), 1.82$ $-1.70(\mathrm{~m}, 2 \mathrm{H}), 1.44-1.26(\mathrm{~m}, 12 \mathrm{H}), 0.92(\mathrm{t}, J=6.8 \mathrm{~Hz}, 9 \mathrm{H}), 0.80(\mathrm{t}, J=6.7 \mathrm{~Hz}, 6 \mathrm{H})$.

${ }^{13} \mathrm{C}\left\{{ }^{1} \mathrm{H}\right\}$ NMR $\left(101 \mathrm{MHz}, \mathrm{CDCl}_{3}\right) \delta 145.6,142.6,128.5,128.3,128.1,125.7,36.3,35.3$, $30.7,27.5,26.5,13.8,12.9$.

HRMS (Cl+) m/z: [M-H] $]^{+}$calcd for $\mathrm{C}_{23} \mathrm{H}_{39} \mathrm{Ge:}$ 389.2264; found: 389.2277 .

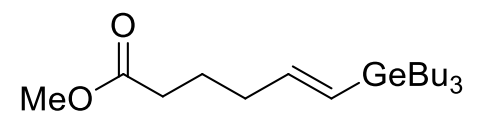

(E)-methyl 6-(tributylgermyl)hex-5-enoate (3c). Prepared according to the general procedure using methyl 5 -hexynoate $(27.8 \mathrm{mg}, 0.22 \mathrm{mmol}, 1.1 \mathrm{eq})$. The crude material was purified by flash silica gel chromatography using $\mathrm{E}_{2} \mathrm{O}$ as the eluent to afford a mixture of $\beta$-vinylgermane $3 \mathrm{c}$ and $\alpha$-vinylgermane $2 \mathrm{c}$ as red-brown oil $(73.3 \mathrm{mg}, 99 \%$ isolated yield). The ratio of $\beta / \alpha$ was determined to be $10: 1$ by the ${ }^{1} \mathrm{H}$ NMR analysis of the isolated mixture.

${ }^{1} \mathrm{H}$ NMR $\left(400 \mathrm{MHz}, \mathrm{CDCl}_{3}\right) \delta 5.85(\mathrm{dt}, J=18.3,5.9 \mathrm{~Hz}, 1 \mathrm{H}), 5.72(\mathrm{~d}, J=18.3 \mathrm{~Hz}, 1 \mathrm{H})$, $3.66(\mathrm{~s}, 3 \mathrm{H}), 2.30$ (t, $J=7.5 \mathrm{~Hz}, 2 \mathrm{H}), 2.14$ (dt, $J=6.3,5.9 \mathrm{~Hz}, 2 \mathrm{H}), 1.77-1.68(\mathrm{~m}, 2 \mathrm{H}), 1.35-$ $1.27(\mathrm{~m}, 12 \mathrm{H}), 0.88(\mathrm{t}, J=6.5 \mathrm{~Hz}, 9 \mathrm{H}), 0.78-0.70(\mathrm{~m}, 6 \mathrm{H})$.

${ }^{13} \mathrm{C}\left\{{ }^{1} \mathrm{H}\right\}$ NMR $\left(101 \mathrm{MHz}, \mathrm{CDCl}_{3}\right) \delta 174.1,144.5,128.9,51.4,36.0,33.3,27.4,26.4,24.0$, 13.7, 12.8 .

HRMS (ES+) m/z: [M+Na] ${ }^{+}$calcd for $\mathrm{C}_{19} \mathrm{H}_{38} \mathrm{GeO}_{2} \mathrm{Na:} 395.1981$; found: 395.1990 .<smiles>COC(=O)/C=C/[14CH3]</smiles>

(E)-methyl 3-(tributylgermyl)acrylate (3d). Prepared according to the general procedure using methyl propiolate $(20.0 \mu \mathrm{L}, 0.22 \mathrm{mmol}, 1.1 \mathrm{eq})$. The crude material was purified by flash silica gel chromatography using $\mathrm{Et}_{2} \mathrm{O}$ as the eluent to afford a mixture of $\beta$ - 
vinylgermane $\mathbf{3} \mathbf{d}$ and $\alpha$-vinylgermane $\mathbf{2} \mathbf{d}$ as orange oil ( $59.6 \mathrm{mg}, 91 \%$ isolated yield). The ratio of $\beta / \alpha$ was determined to be $1: 1.4$ by the ${ }^{1} \mathrm{H}$ NMR analysis of the isolated mixture.

${ }^{1} \mathrm{H}$ NMR $\left(400 \mathrm{MHz}, \mathrm{CDCl}_{3}\right) \delta 7.47(\mathrm{~d}, J=18.8 \mathrm{~Hz}, 1 \mathrm{H}), 6.22(\mathrm{~d}, J=18.8 \mathrm{~Hz}, 1 \mathrm{H}), 3.74(\mathrm{~s}$, $3 \mathrm{H}), 1.43-1.26(\mathrm{~m}, 12 \mathrm{H}$, merged), $0.93-0.75(\mathrm{~m}, 15 \mathrm{H}$, merged).

${ }^{13} \mathrm{C}\left\{{ }^{1} \mathrm{H}\right\}$ NMR $\left(101 \mathrm{MHz}, \mathrm{CDCl}_{3}\right) \delta 161.8,143.7,128.9,52.6,27.2,26.4,13.7,12.6$.

HRMS (ES+) m/z: [M+H] $]^{+}$calcd for $\mathrm{C}_{16} \mathrm{H}_{33} \mathrm{GeO}_{2}: 331.1692$; found: 331.1695 .

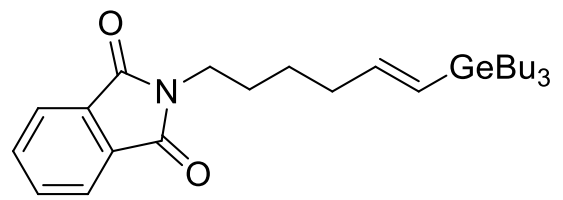

(E)-2-(6-(tributylgermyl)hex-5-en-1-yl)isoindoline-1,3-dione (3e). Prepared according to the general procedure using $\mathrm{N}$-(hex-5-ynyl)-phthalimide $(50.0 \mathrm{mg}, 0.22 \mathrm{mmol}, 1.1 \mathrm{eq})$. The crude material was purified by flash silica gel chromatography using $\mathrm{Et}_{2} \mathrm{O}$ as the eluent to afford a mixture of $\beta$-vinylgermane $3 e$ and $\alpha$-vinylgermane $2 e$ as dark red oil $(87.5 \mathrm{mg}$, $93 \%$ isolated yield). The ratio of $\beta / \alpha$ was determined to be $11: 1$ by the ${ }^{1} \mathrm{H}$ NMR analysis of the isolated mixture.

${ }^{1} \mathrm{H}$ NMR $\left(400 \mathrm{MHz}, \mathrm{CDCl}_{3}\right) \delta 7.82(\mathrm{dd}, J=5.4,3.1 \mathrm{~Hz}, 2 \mathrm{H}), 7.68(\mathrm{dd}, J=5.4,3.1 \mathrm{~Hz}, 2 \mathrm{H})$, 5.85 (dt, $J=18.3,6.3 \mathrm{~Hz}, 1 \mathrm{H}$ ), $5.68(\mathrm{~d}, J=18.3 \mathrm{~Hz}, 1 \mathrm{H}$ ), 3.67 (t, $J=7.2 \mathrm{~Hz}, 2 \mathrm{H}), 2.14$ (dt, $J=6.9$, $6.3 \mathrm{~Hz}, 2 \mathrm{H}), 1.74-1.61(\mathrm{~m}, 2 \mathrm{H}), 1.48-1.39(\mathrm{~m}, 2 \mathrm{H}), 1.34-1.24(\mathrm{~m}, 12 \mathrm{H}), 0.85(\mathrm{t}, J=6.8 \mathrm{~Hz}$, $9 \mathrm{H}), 0.72(\mathrm{t}, J=6.8 \mathrm{~Hz}, 6 \mathrm{H})$.

${ }^{13} \mathrm{C}\left\{{ }^{1} \mathrm{H}\right\}$ NMR $\left(101 \mathrm{MHz}, \mathrm{CDCl}_{3}\right) \delta 168.3,145.2,133.8,132.2,128.2,123.1,37.9,36.2$, 28.1, 27.4, 26.4, 26.1, 13.7, 12.8 .

HRMS (ES+) m/z: [M-H] $]^{+}$calcd for $\mathrm{C}_{26} \mathrm{H}_{40} \mathrm{GeNO}_{2}: 472.2271$; found: 472.2278 .

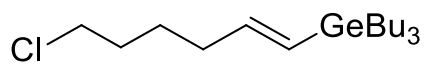

(E)-tributyl(6-chlorohex-1-en-1-yl)germane (3f). Prepared according to the general procedure using 6-chloro-1-hexyne $(27.0 \mu \mathrm{L}, 0.22 \mathrm{mmol}, 1.1 \mathrm{eq})$. The crude material was purified by flash silica gel chromatography using $\mathrm{Et}_{2} \mathrm{O}$ as the eluent to afford a mixture of $\beta$-vinylgermane $\mathbf{3 f}$ and $\alpha$-vinylgermane $\mathbf{2 f}$ as dark red oil (70.3 $\mathrm{mg}, \mathbf{9 7 \%}$ isolated yield). The ratio of $\beta / \alpha$ was determined to be $8.5: 1$ by the ${ }^{1} \mathrm{H}$ NMR analysis of the isolated mixture.

${ }^{1} \mathrm{H}$ NMR $\left(400 \mathrm{MHz}, \mathrm{CDCl}_{3}\right) \delta 5.88(\mathrm{dt}, J=18.3,6.1 \mathrm{~Hz}, 1 \mathrm{H}), 5.72(\mathrm{~d}, J=18.3 \mathrm{~Hz}, 1 \mathrm{H})$, $3.54(\mathrm{t}, J=6.7 \mathrm{~Hz}, 2 \mathrm{H}), 2.22-2.09(\mathrm{~m}, 2 \mathrm{H}), 1.83-1.73(\mathrm{~m}, 2 \mathrm{H}), 1.60-1.50(\mathrm{~m}, 2 \mathrm{H}), 1.37-1.26$ $(\mathrm{m}, 12 \mathrm{H}), 0.89(\mathrm{t}, J=6.8 \mathrm{~Hz}, 9 \mathrm{H}), 0.81-0.69(\mathrm{~m}, 6 \mathrm{H})$. 
${ }^{13} \mathrm{C}\left\{{ }^{1} \mathrm{H}\right\}$ NMR $\left(101 \mathrm{MHz}, \mathrm{CDCl}_{3}\right) \delta 145.1,128.4,44.9,35.9,32.0,27.4,26.5,26.0,13.8$, 12.9 .

HRMS (Cl+) m/z: [M-H] ${ }^{+}$calcd for $\mathrm{C}_{18} \mathrm{H}_{36} \mathrm{GeCl}: 361.1717$; found: 361.1727 .

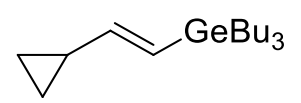

(E)-tributyl(2-cyclopropylvinyl)germane (3g). Prepared according to the general procedure using cyclopropylacetylene $(19.0 \mu \mathrm{L}, 0.22 \mathrm{mmol}, 1.1 \mathrm{eq})$. The crude material was purified by flash silica gel chromatography using $\mathrm{Et}_{2} \mathrm{O}$ as the eluent to afford a mixture of $\beta$ vinylgermane $\mathbf{3 g}$ and $\alpha$-vinylgermane $\mathbf{2} \mathbf{g}$ as dark brown oil (56.8 $\mathrm{mg}, 91 \%$ isolated yield). The ratio of $\beta / \alpha$ was determined to be $1.7: 1$ by the ${ }^{1} \mathrm{H}$ NMR analysis of the isolated mixture.

${ }^{1} \mathrm{H}$ NMR $\left(400 \mathrm{MHz}, \mathrm{CDCl}_{3}\right) \delta 5.77(\mathrm{~d}, J=18.3 \mathrm{~Hz}, 1 \mathrm{H}), 5.38$ (dd, $1 \mathrm{H}$, merged), $1.49-1.42$ $(\mathrm{m}, 1 \mathrm{H}), 1.38-1.29(\mathrm{~m}, 12 \mathrm{H}), 0.89(\mathrm{~m}, 9 \mathrm{H}), 0.86-0.82(\mathrm{~m}, 2 \mathrm{H}), 0.80-0.60(\mathrm{~m}, 6 \mathrm{H}), 0.48-0.37$ $(\mathrm{m}, 2 \mathrm{H})$.

${ }^{13} \mathrm{C}\left\{{ }^{1} \mathrm{H}\right\}$ NMR $\left(101 \mathrm{MHz}, \mathrm{CDCl}_{3}\right) \delta$ 149.3, 124.3, 27.4, 26.5, 18.8, 17.2, 13.7, 12.9, 7.0.

HRMS (El+) m/z: [M] ${ }^{+}$calcd for $\mathrm{C}_{17} \mathrm{H}_{34} \mathrm{Ge}: 312.1872$; found: 312.1862 .<smiles>CCCCOC=CC1CCCCC1</smiles>

(E)-tributyl(2-cyclohexylvinyl)germane (3h). Prepared according to the general procedure using cyclohexylacetylene $(29.0 \mu \mathrm{L}, 0.22 \mathrm{mmol}, 1.1 \mathrm{eq})$. The crude material was purified by flash silica gel chromatography using $\mathrm{Et}_{2} \mathrm{O}$ as the eluent to afford a mixture of $\beta$ vinylgermane $3 \mathbf{h}$ and $\alpha$-vinylgermane $\mathbf{2} \mathbf{h}$ as dark red oil $(70.1 \mathrm{mg}, 99 \%$ isolated yield). The ratio of $\beta / \alpha$ was determined to be $48: 1$ by the ${ }^{1} \mathrm{H}$ NMR analysis of the isolated mixture.

${ }^{1} \mathbf{H}$ NMR $\left(400 \mathrm{MHz}, \mathrm{CDCl}_{3}\right) \delta 5.85$ (dd, $\left.J=18.5,6.0 \mathrm{~Hz}, 1 \mathrm{H}\right), 5.64(\mathrm{dd}, J=18.5,1.1 \mathrm{~Hz}$, $1 \mathrm{H}), 2.05-1.90(\mathrm{~m}, 1 \mathrm{H}), 1.78-1.68(\mathrm{~m}, 4 \mathrm{H}), 1.66-1.57(\mathrm{~m}, 1 \mathrm{H}), 1.38-1.27(\mathrm{~m}, 12 \mathrm{H}), 1.27-$ $1.24(\mathrm{~m}, 1 \mathrm{H}), 1.24-1.01(\mathrm{~m}, 4 \mathrm{H}), 0.89(\mathrm{t}, J=6.9 \mathrm{~Hz}, 9 \mathrm{H}), 0.79-0.70(\mathrm{~m}, 6 \mathrm{H})$.

${ }^{13} \mathrm{C}\left\{{ }^{1} \mathrm{H}\right\}$ NMR $\left(101 \mathrm{MHz}, \mathrm{CDCl}_{3}\right) \delta 151.8,123.8,44.1,32.6,27.4,26.5,26.3,26.113 .8$, 12.9.

HRMS (El+) m/z: [M] ${ }^{+}$calcd for $\mathrm{C}_{20} \mathrm{H}_{40} \mathrm{Ge}: 354.2342$; found: 354.2336 . 


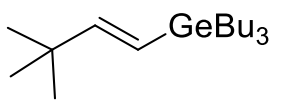

(E)-tributyl(3,3-dimethylbut-1-en-1-yl)germane (3i). Prepared according to the general procedure using 3,3-dimethyl-1-butyne $(27.0 \mu \mathrm{L}, 0.22 \mathrm{mmol}, 1.1 \mathrm{eq})$. The crude material was purified by flash silica gel chromatography using $\mathrm{Et}_{2} \mathrm{O}$ as the eluent to afford a mixture of $\beta$-vinylgermane $3 \mathbf{i}$ and $\alpha$-vinylgermane $2 \boldsymbol{i}$ as dark red oil $(55.7 \mathrm{mg}, 85 \%$ isolated yield). The ratio of $\beta / \alpha$ was determined to be $43: 1$ by the ${ }^{1} \mathrm{H}$ NMR analysis of the isolated mixture.

${ }^{1} \mathrm{H}$ NMR $\left(400 \mathrm{MHz}, \mathrm{CDCl}_{3}\right) \delta 5.91(\mathrm{~d}, J=18.7 \mathrm{~Hz}, 1 \mathrm{H}), 5.58(\mathrm{~d}, J=18.7 \mathrm{~Hz}, 1 \mathrm{H}), 1.37-$ $1.30(\mathrm{~m}, 12 \mathrm{H}), 1.00(\mathrm{~s}, 9 \mathrm{H}), 0.89(\mathrm{t}, J=7.0 \mathrm{~Hz}, 9 \mathrm{H}), 0.79-0.72(\mathrm{~m}, 6 \mathrm{H})$.

${ }^{13} \mathrm{C}\left\{{ }^{1} \mathrm{H}\right\}$ NMR $\left(101 \mathrm{MHz}, \mathrm{CDCl}_{3}\right) \delta 156.5,120.2,35.1,29.2,27.4,26.4,13.8,12.9$.

HRMS (Cl+) m/z: [M-H] ${ }^{+}$calcd for $\mathrm{C}_{18} \mathrm{H}_{37} \mathrm{Ge:}$ 327.2107; found: 327.2095.

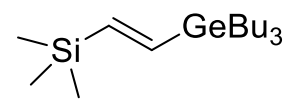

(E)-trimethyl(2-(tributylgermyl)vinyl)silane (3j). Prepared according to the general procedure using ethynyltrimethylsilane $(30.5 \mu \mathrm{L}, 0.22 \mathrm{mmol}, 1.1 \mathrm{eq})$. The crude material was purified by flash silica gel chromatography using $\mathrm{Et}_{2} \mathrm{O}$ as the eluent to afford $\beta$ vinylgermane $3 \mathbf{j}$ as dark red oil ( $64.9 \mathrm{mg}, 95 \%$ isolated yield). No traces of $\alpha$-vinylgermane 2j were found by the ${ }^{1} \mathrm{H}$ NMR analysis of the isolated compound.

${ }^{1} \mathrm{H}$ NMR $\left(400 \mathrm{MHz}, \mathrm{CDCl}_{3}\right) \delta 6.70(\mathrm{~d}, J=22.1 \mathrm{~Hz}, 1 \mathrm{H}), 6.48(\mathrm{~d}, J=22.1 \mathrm{~Hz}, 1 \mathrm{H}), 1.37-$ $1.31(\mathrm{~m}, 12 \mathrm{H}), 0.89(\mathrm{t}, J=7.0 \mathrm{~Hz}, 9 \mathrm{H}), 0.81-0.76(\mathrm{~m}, 6 \mathrm{H}), 0.07(\mathrm{~s}, 9 \mathrm{H})$.

${ }^{13} \mathrm{C}\left\{{ }^{1} \mathrm{H}\right\}$ NMR $\left(101 \mathrm{MHz}, \mathrm{CDCl}_{3}\right) \delta 150.0,148.9,27.4,26.5,13.8,12.6,-1.5$.

HRMS (El+) m/z: [M] ${ }^{+}$calcd for $\mathrm{C}_{17} \mathrm{H}_{38} \mathrm{GeSi}$ : 344.1955; found: 344.1963 .

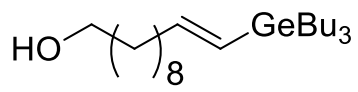

(E)-11-(tributylgermyl)undec-10-en-1-ol (3k). Prepared according to the general procedure using 10-undecyn-1-ol ( $42.0 \mu \mathrm{L}, 0.22 \mathrm{mmol}, 1.1 \mathrm{eq})$. The crude material was purified by flash silica gel chromatography using $\mathrm{Et}_{2} \mathrm{O}$ as the eluent to afford a mixture of $\beta$ vinylgermane $\mathbf{3 k}$ and $\boldsymbol{\alpha}$-vinylgermane $\mathbf{2} \mathbf{k}$ as dark red oil ( $81.9 \mathrm{mg}, 99 \%$ isolated yield). The ratio of $\beta / \alpha$ was determined to be $10: 1$ by the ${ }^{1} \mathrm{H}$ NMR analysis of the isolated mixture.

${ }^{1} \mathrm{H}$ NMR $\left(400 \mathrm{MHz}, \mathrm{CDCl}_{3}\right) \delta 5.88(\mathrm{dt}, J=18.3,6.3 \mathrm{~Hz}, 1 \mathrm{H}), 5.67(\mathrm{dt}, J=18.3,1.2 \mathrm{~Hz}$, $1 \mathrm{H}), 3.62(\mathrm{t}, J=6.6 \mathrm{~Hz}, 2 \mathrm{H}), 2.13-2.06(\mathrm{~m}, 2 \mathrm{H}), 1.60-1.52(\mathrm{~m}, 2 \mathrm{H}), 1.35-1.27(\mathrm{~m}, 24 \mathrm{H}$, merged), $0.88(\mathrm{t}, J=7.1 \mathrm{~Hz}, 9 \mathrm{H}), 0.76-0.71(\mathrm{~m}, 6 \mathrm{H})$. 
${ }^{13} \mathrm{C}\left\{{ }^{1} \mathrm{H}\right\}$ NMR $\left(101 \mathrm{MHz}, \mathrm{CDCl}_{3}\right) \delta 146.2,127.3,63.0,36.8,32.8,29.6,29.4,29.4,29.1$, 28.9, 27.4, 26.5, 25.7, 13.7, 12.9 .

HRMS (ES+) m/z: $[\mathrm{M}+\mathrm{Na}]^{+}$calcd for $\mathrm{C}_{23} \mathrm{H}_{48} \mathrm{GeONa:} 437.2815$; found: 437.2827 .

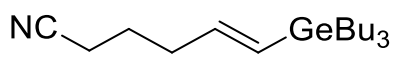

(E)-6-(tributylgermyl)hex-5-enenitrile (3I). Prepared according to the general procedure using 5-hexynenitrile $(23.0 \mu \mathrm{L}, 0.22 \mathrm{mmol}, 1.1 \mathrm{eq})$. The crude material was purified by flash silica gel chromatography using $\mathrm{Et}_{2} \mathrm{O}$ as the eluent to afford a mixture of $\beta$ vinylgermane $3 \mathbf{I}$ and $\alpha$-vinylgermane $2 \mathrm{I}$ as dark red oil ( $67.4 \mathrm{mg}, 99 \%$ isolated yield). The ratio of $\beta / \alpha$ was determined to be $9: 1$ by the ${ }^{1} \mathrm{H}$ NMR analysis of the isolated mixture.

${ }^{1} \mathrm{H}$ NMR $\left(400 \mathrm{MHz}, \mathrm{CDCl}_{3}\right) \delta 5.81$ (s, 2H, merged), 2.32 (t, $J=6.8 \mathrm{~Hz}, 2 \mathrm{H}$, merged), 2.29 $-2.23(\mathrm{~m}, 2 \mathrm{H}$, merged), $1.80-1.73(\mathrm{~m}, 2 \mathrm{H}), 1.36-1.27(\mathrm{~m}, 12 \mathrm{H}), 0.91-0.85(\mathrm{~m}, 9 \mathrm{H}), 0.79-$ $0.72(\mathrm{~m}, 6 \mathrm{H})$.

${ }^{1} \mathbf{H}$ NMR $(400 \mathrm{MHz}$, acetone-d6) $\delta 5.97(\mathrm{dt}, J=18.4,6.4 \mathrm{~Hz}, 1 \mathrm{H}), 5.85(\mathrm{~d}, J=18.4 \mathrm{~Hz}$, $1 \mathrm{H}), 2.44(\mathrm{t}, J=7.2 \mathrm{~Hz}, 2 \mathrm{H}), 2.28(\mathrm{dt}, J=7.0,6.4 \mathrm{~Hz}, 2 \mathrm{H}), 1.80-1.72(\mathrm{~m}, 2 \mathrm{H}), 1.43-1.27(\mathrm{~m}$, $12 \mathrm{H}), 0.89(\mathrm{t}, J=6.9 \mathrm{~Hz}, 9 \mathrm{H}), 0.84-0.79(\mathrm{~m}, 6 \mathrm{H})$.

${ }^{13} \mathrm{C}\left\{{ }^{1} \mathrm{H}\right\}$ NMR $\left(101 \mathrm{MHz}, \mathrm{CDCl}_{3}\right) \delta$ 142.6, 130.8, 119.6, 35.2, 27.4, 26.4, 24.5, 16.3, 13.7, 12.8 .

HRMS (Cl+) m/z: $[\mathrm{M}+\mathrm{H}]^{+}$calcd for $\mathrm{C}_{18} \mathrm{H}_{36} \mathrm{GeN}$ : 340.2060; found: 340.2066 .

TBSO $\mathrm{GeBu}_{3}$

(E)-tert-butyldimethyl((5-(tributylgermyl)pent-4-en-1-yl)oxy)silane $\quad(3 \mathrm{~m}) . \quad$ Prepared according to the general procedure using 1-(tert-butyldimethylsilyloxy)-4-pentyne (44.0 $\mathrm{mg}, 0.22 \mathrm{mmol}, 1.1 \mathrm{eq})$. The crude material was purified by flash silica gel chromatography using $\mathrm{Et}_{2} \mathrm{O}$ as the eluent to afford a mixture of $\beta$-vinylgermane $3 \mathrm{~m}$ and $\alpha$-vinylgermane $2 \mathrm{~m}$ as dark red oil ( $88.3 \mathrm{mg}, 99 \%$ isolated yield). The ratio of $\beta / \alpha$ was determined to be $10: 1$ by the ${ }^{1} \mathrm{H}$ NMR analysis of the isolated mixture.

${ }^{1} \mathrm{H}$ NMR $\left(400 \mathrm{MHz}, \mathrm{CDCl}_{3}\right) \delta 5.90(\mathrm{dt}, J=18.4,6.4 \mathrm{~Hz}, 1 \mathrm{H}), 5.71(\mathrm{~d}, J=18.4 \mathrm{~Hz}, 1 \mathrm{H})$, $3.61(\mathrm{t}, J=6.8 \mathrm{~Hz}, 2 \mathrm{H}$ ), 2.16 (dt, $J=6.8,6.4 \mathrm{~Hz}, 2 \mathrm{H}$ ), 1.62 (quin., $J=6.8 \mathrm{~Hz}, 2 \mathrm{H}$ ), $1.35-1.30(\mathrm{~m}$, $12 \mathrm{H}), 0.90(\mathrm{~s}, 9 \mathrm{H}), 0.89-0.86(\mathrm{~m}, 9 \mathrm{H}), 0.78-0.72(\mathrm{~m}, 6 \mathrm{H}), 0.05(\mathrm{~s}, 6 \mathrm{H})$.

${ }^{13} \mathrm{C}\left\{{ }^{1} \mathrm{H}\right\}$ NMR $\left(101 \mathrm{MHz}, \mathrm{CDCl}_{3}\right) \delta 145.4,127.8,62.5,32.9,32.0,27.4,26.5,26.0,18.3$, 13.7, 12.9, -5.3 .

HRMS (Cl+) m/z: $[\mathrm{M}-\mathrm{H}]^{+}$calcd for $\mathrm{C}_{23} \mathrm{H}_{49} \mathrm{GeOSi}$ : 443.2764; found: 443.2774 . 


$$
\mathrm{Ph} \curvearrowright \mathrm{GeBu}_{3}
$$

(E)-tributyl(styryl)germane (3n). Prepared according to the general procedure using phenylacetylene $(24.5 \mu \mathrm{L}, 0.22 \mathrm{mmol}, 1.1 \mathrm{eq})$. The crude material was purified by flash silica gel chromatography using $\mathrm{Et}_{2} \mathrm{O}$ as the eluent to afford a mixture of $\beta$-vinylgermane $3 \mathrm{n}$ and $\alpha$-vinylgermane $\mathbf{2 n}$ as dark red oil $(66.9 \mathrm{mg}, 96 \%$ isolated yield). The ratio of $\beta / \alpha$ was determined to be $6.3: 1$ by the ${ }^{1} \mathrm{H}$ NMR analysis of the isolated mixture.

${ }^{1} \mathbf{H}$ NMR $\left(400 \mathrm{MHz}, \mathrm{CDCl}_{3}\right) \delta 7.55-7.41(\mathrm{~m}, 2 \mathrm{H}), 7.40-7.30(\mathrm{~m}, 2 \mathrm{H}), 7.29$ - $7.22(\mathrm{~m}, 1 \mathrm{H})$, $6.85(\mathrm{~d}, J=19.0 \mathrm{~Hz}, 1 \mathrm{H}), 6.66(\mathrm{~d}, J=19.0 \mathrm{~Hz}, 1 \mathrm{H}), 1.47-1.34(\mathrm{~m}, 12 \mathrm{H}), 0.97-0.86(\mathrm{~m}, 15 \mathrm{H})$.

${ }^{13} \mathrm{C}\left\{{ }^{1} \mathrm{H}\right\}$ NMR $\left(101 \mathrm{MHz}, \mathrm{CDCl}_{3}\right) \delta 142.9,138.5,129.0,128.5,127.6,126.2,27.5,26.6$, 13.8, 13.0.

HRMS (El+) m/z: [M] ${ }^{+}$calcd for $\mathrm{C}_{20} \mathrm{H}_{34} \mathrm{Ge:} 348.1872$; found: 348.1878.

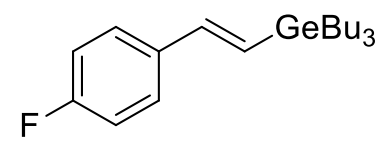

(E)-tributyl(4-fluorostyryl)germane (30). Prepared according to the general procedure using 4-fluorophenylacetylene $(25.0 \mu \mathrm{L}, 0.22 \mathrm{mmol}, 1.1 \mathrm{eq})$. The crude material was purified by flash silica gel chromatography using $\mathrm{Et}_{2} \mathrm{O}$ as the eluent to afford a mixture of $\beta$-vinylgermane 30 and $\alpha$-vinylgermane 20 as dark brown oil $(67.9 \mathrm{mg}, 93 \%$ isolated yield). The ratio of $\beta / \alpha$ was determined to be $7.3: 1$ by the ${ }^{1} \mathrm{H}$ NMR analysis of the isolated mixture.

${ }^{1} \mathrm{H}$ NMR $\left(400 \mathrm{MHz}, \mathrm{CDCl}_{3}\right) \delta 7.39(\mathrm{~m}, 2 \mathrm{H}), 7.02(\mathrm{~m}, 2 \mathrm{H}), 6.77(\mathrm{~d}, J=18.9 \mathrm{~Hz}, 1 \mathrm{H}), 6.54$ $(\mathrm{d}, J=18.9 \mathrm{~Hz}, 1 \mathrm{H}), 1.44-1.34(\mathrm{~m}, 12 \mathrm{H}), 0.94-0.90(\mathrm{~m}, 9 \mathrm{H}), 0.89-0.85(\mathrm{~m}, 6 \mathrm{H})$.

${ }^{13} \mathrm{C}\left\{{ }^{1} \mathrm{H}\right\}$ NMR $\left(101 \mathrm{MHz}, \mathrm{CDCl}_{3}\right) \delta 163.6,141.6,127.7,127.6,115.4,115.2,27.4,26.5$, 13.8, 12.9.

HRMS (El+) m/z: [M] calcd for $\mathrm{C}_{20} \mathrm{H}_{33} \mathrm{GeF}$ : 366.1778; found: 366.1786.<smiles>COc1ccc(/C=C/C(C)(C)C)cc1</smiles>

(E)-tributyl(4-methoxystyryl)germane (3p). Prepared according to the general procedure using 4-methoxyphenylacetylene $(28.5 \mu \mathrm{L}, 0.22 \mathrm{mmol}, 1.1 \mathrm{eq})$. The crude material was purified by flash silica gel chromatography using $\mathrm{Et}_{2} \mathrm{O}$ as the eluent to afford a mixture of $\beta$-vinylgermane $3 p$ and $\alpha$-vinylgermane $2 p$ as dark brown oil $(75.1 \mathrm{mg}, 99 \%$ isolated yield). The ratio of $\beta / \alpha$ was determined to be $8.1: 1$ by the ${ }^{1} \mathrm{H}$ NMR analysis of the isolated mixture. 
${ }^{1} \mathrm{H}$ NMR $\left(400 \mathrm{MHz}, \mathrm{CDCl}_{3}\right) \delta 7.38(\mathrm{~m}, 2 \mathrm{H}), 6.88(\mathrm{~m}, 2 \mathrm{H}), 6.76(\mathrm{~d}, J=18.9 \mathrm{~Hz}, 1 \mathrm{H}), 6.46$ (d, $J=18.9 \mathrm{~Hz}, 1 \mathrm{H}), 3.82(\mathrm{~s}, 3 \mathrm{H}), 1.43-1.33(\mathrm{~m}, 12 \mathrm{H}), 0.92(\mathrm{~m}, 9 \mathrm{H}), 0.89-0.85(\mathrm{~m}, 6 \mathrm{H})$.

${ }^{13} \mathrm{C}\left\{{ }^{1} \mathrm{H}\right\}$ NMR $\left(101 \mathrm{MHz}, \mathrm{CDCl}_{3}\right) \delta 159.3,142.3,131.6,127.3,126.0,113.9,55.3,27.4$, 26.5, 13.8, 13.0.

HRMS (El+) m/z: [M] calcd for $\mathrm{C}_{21} \mathrm{H}_{36} \mathrm{GeO}: 378.1978$; found: 378.1980 .

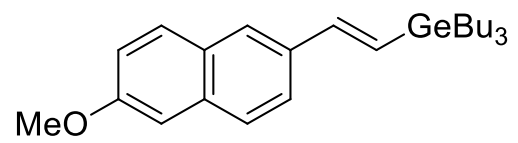

(E)-tributyl(2-(6-methoxynaphthalen-2-yl)vinyl)germane (3q). Prepared according to the general procedure using 2-ethynyl-6-methoxynaphthalene (40.1 mg, $0.22 \mathrm{mmol}, 1.1 \mathrm{eq})$. The crude material was purified by flash silica gel chromatography using $\mathrm{Et}_{2} \mathrm{O}$ as the eluent to afford a mixture of $\beta$-vinylgermane $\mathbf{3 q}$ and $\alpha$-vinylgermane $\mathbf{2 q}$ as brown oil (83.9 $\mathrm{mg}, 98 \%$ isolated yield). The ratio of $\beta / \alpha$ was determined to be $2.3: 1$ by the ${ }^{1} \mathrm{H}$ NMR analysis of the isolated mixture.

${ }^{1} \mathrm{H}$ NMR $\left(400 \mathrm{MHz}, \mathrm{CDCl}_{3}\right) \delta 7.76-7.65(\mathrm{~m}, 4 \mathrm{H}), 7.16-7.12(\mathrm{~m}, 2 \mathrm{H}), 6.98(\mathrm{~d}, J=18.9$ $\mathrm{Hz}, 1 \mathrm{H}), 6.72(\mathrm{~d}, J=18.9 \mathrm{~Hz}, 1 \mathrm{H}), 3.93(\mathrm{~s}, 3 \mathrm{H}), 1.48-1.37(\mathrm{~m}, 12 \mathrm{H}), 0.97-0.93(\mathrm{~m}, 9 \mathrm{H}), 0.92$ $-0.84(\mathrm{~m}, 6 \mathrm{H})$.

${ }^{13} \mathrm{C}\left\{{ }^{1} \mathrm{H}\right\}$ NMR $\left(101 \mathrm{MHz}, \mathrm{CDCl}_{3}\right) \delta 157.7,143.0,134.3,134.0,129.6,129.1,128.1,126.9$, 126.0, 123.9, 118.9, 105.9, 55.3, 27.5, 26.6, 13.8, 13.1.

HRMS (ES+) m/z: $[\mathrm{M}+\mathrm{H}]^{+}$calcd for $\mathrm{C}_{25} \mathrm{H}_{39} \mathrm{GeO}: 429.2213$; found: 429.2207.

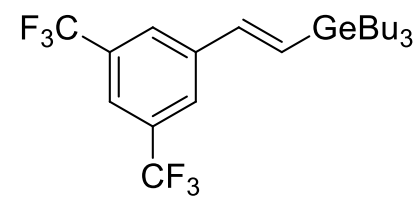

(E)-(3,5-bis(trifluoromethyl)styryl)tributylgermane (3r). Prepared according to the general procedure using 1-ethynyl-3,5-bis(trifluoromethyl)benzene (39.0 $\mu \mathrm{L}, 0.22 \mathrm{mmol}, 1.1 \mathrm{eq}$ ). The crude material was purified by flash silica gel chromatography using $\mathrm{Et}_{2} \mathrm{O}$ as the eluent to afford a mixture of $\beta$-vinylgermane $3 r$ and $\alpha$-vinylgermane $2 r$ as dark ruby oil (94.4 mg, $98 \%$ isolated yield). The ratio of $\beta / \alpha$ was determined to be $3.8: 1$ by the ${ }^{1} \mathrm{H}$ NMR analysis of the isolated mixture.

${ }^{1} \mathrm{H}$ NMR $\left(400 \mathrm{MHz}, \mathrm{CDCl}_{3}\right) \delta 7.85-7.79(\mathrm{~m}, 2 \mathrm{H}), 7.74-7.71(\mathrm{~m}, 1 \mathrm{H}), 6.86(\mathrm{~s}, 2 \mathrm{H}$, merged), $1.43-1.32(\mathrm{~m}, 12 \mathrm{H}), 0.95-0.91(\mathrm{~m}, 9 \mathrm{H}), 0.91-0.86(\mathrm{~m}, 6 \mathrm{H})$.

${ }^{1} \mathrm{H}$ NMR $(400 \mathrm{MHz}$, acetone- $d 6) \delta 8.18-8.08(\mathrm{~m}, 2 \mathrm{H}), 7.88-7.80(\mathrm{~m}, 1 \mathrm{H}), 7.17(\mathrm{~d}, J=$ $19.1 \mathrm{~Hz}, 1 \mathrm{H}$, partially merged), $7.11(\mathrm{~d}, J=19.1 \mathrm{~Hz}, 1 \mathrm{H}$, partially merged), $1.49-1.41(\mathrm{~m}, 5 \mathrm{H})$, $1.40-1.32(\mathrm{~m}, 7 \mathrm{H}), 0.98-0.86(\mathrm{~m}, 15 \mathrm{H})$. 
${ }^{13} \mathrm{C}\left\{{ }^{1} \mathrm{H}\right\}$ NMR $\left(101 \mathrm{MHz}, \mathrm{CDCl}_{3}\right) \delta 140.3,139.9,135.0,125.9,124.8,122.0,27.3,26.4$, 13.7, 12.8 .

HRMS (Cl+) m/z: [M+H] calcd for $\mathrm{C}_{22} \mathrm{H}_{33} \mathrm{GeF}_{6}: 485.1698$; found: 485.1714 .

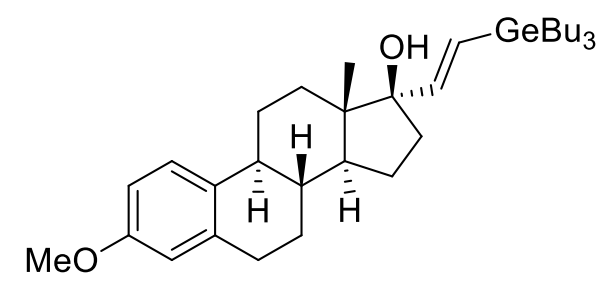

(8R,9S,13S,14S,17R)-3-methoxy-13-methyl-17-((E)-2-(tributylgermyl)vinyl)-

7,8,9,11,12,13,14,15,16,17- decahydro-6H-cyclopenta[a]phenanthren-17-ol (3s). Prepared according to the general procedure using Mestranol ( $68.3 \mathrm{mg}, 0.22 \mathrm{mmol}, 1.1 \mathrm{eq})$ in $3 \mathrm{~mL}$ of DCE. The crude material was purified by flash silica gel chromatography using $\mathrm{Et}_{2} \mathrm{O}$ as the eluent to afford $\beta$-vinylgermane $3 s$ as dark red oil ( $110.6 \mathrm{mg}, 99 \%$ isolated yield). No traces of $\alpha$-vinylgermane $2 s$ were found by the ${ }^{1} \mathrm{H}$ NMR analysis of the isolated compound.

${ }^{1} \mathrm{H}$ NMR $\left(400 \mathrm{MHz}, \mathrm{CDCl}_{3}\right) \delta 7.19(\mathrm{~d}, J=8.4 \mathrm{~Hz}, 1 \mathrm{H}), 6.70(\mathrm{~d}, J=8.4 \mathrm{~Hz}, 1 \mathrm{H}), 6.63(\mathrm{~s}$, $1 \mathrm{H}), 6.16(\mathrm{~d}, J=18.8 \mathrm{~Hz}, 1 \mathrm{H}), 5.88(\mathrm{~d}, J=18.8 \mathrm{~Hz}, 1 \mathrm{H}), 3.77(\mathrm{~s}, 3 \mathrm{H}), 2.90-2.81(\mathrm{~m}, 2 \mathrm{H}), 2.34$ $-2.23(\mathrm{~m}, 1 \mathrm{H}), 2.16-1.97(\mathrm{~m}, 2 \mathrm{H}), 1.96-1.82(\mathrm{~m}, 2 \mathrm{H}), 1.80-1.66(\mathrm{~m}, 2 \mathrm{H}), 1.64-1.56(\mathrm{~m}$, $1 \mathrm{H}), 1.51-1.42(\mathrm{~m}, 5 \mathrm{H}), 1.39-1.32(\mathrm{~m}, 12 \mathrm{H}), 0.95(\mathrm{~s}, 3 \mathrm{H}), 0.92-0.87(\mathrm{~m}, 9 \mathrm{H}), 0.85-0.80(\mathrm{~m}$, $6 \mathrm{H})$.

${ }^{13} \mathrm{C}\left\{{ }^{1} \mathrm{H}\right\}$ NMR $\left(101 \mathrm{MHz}, \mathrm{CDCl}_{3}\right) \delta 157.4,149.5,137.9,132.7,126.3,124.9,113.8,111.4$, 85.1, 55.1, 49.0, 46.7, 43.9, 39.5, 36.0, 32.3, 29.8, 27.5, 27.4 (merged with 27.5), 26.5, 26.4 (merged with 26.5), 23.4, 14.2, 13.8, 13.0 .

HRMS (ES+) m/z: [M-H] ${ }^{+}$calcd for $\mathrm{C}_{33} \mathrm{H}_{53} \mathrm{GeO}_{2}: 555.3257$; found: 555.3264 .

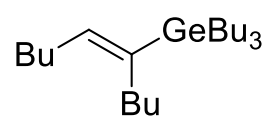

(E)-tributyl(dec-5-en-5-yl)germane (3t). Prepared according to the general procedure using 5-decyne ( $40.0 \mu \mathrm{L}, 0.22 \mathrm{mmol}, 1.1 \mathrm{eq})$. The crude material was purified by flash silica gel chromatography using $\mathrm{Et}_{2} \mathrm{O}$ as the eluent to afford $E$-vinylgermane $\mathbf{3 t}$ as yellow-orange oil (78.2 mg, 102\% isolated yield, some 5-decyne remained in the mixture).

${ }^{1} \mathrm{H} \mathrm{NMR}\left(400 \mathrm{MHz}, \mathrm{CDCl}_{3}\right) \delta 5.49(\mathrm{t}, J=6.9 \mathrm{~Hz}, 1 \mathrm{H}), 2.15-2.07(\mathrm{~m}, 4 \mathrm{H}), 1.35-1.39(\mathrm{~m}$, $20 \mathrm{H}), 0.91-0.87(\mathrm{~m}, 15 \mathrm{H}), 0.78-0.73(\mathrm{~m}, 6 \mathrm{H})$.

${ }^{13} \mathrm{C}\left\{{ }^{1} \mathrm{H}\right\}$ NMR $\left(101 \mathrm{MHz}, \mathrm{CDCl}_{3}\right) \delta 140.0,138.7,32.2,32.0,30.8,27.9,27.4,26.6,22.9$, 22.4, 17.6, 14.0, 13.7, 12.6 . 
HRMS (EI+) m/z: [M] ${ }^{+}$calcd for $\mathrm{C}_{22} \mathrm{H}_{46} \mathrm{Ge}: 384.2811$; found: 384.2819.

$$
\gamma_{7} \mathrm{GePh}_{3}
$$

(E)-dec-1-en-1-yltriphenylgermane (3u). Prepared according to the general procedure using 1-decyne (40.0 $\mu \mathrm{L}, 0.22 \mathrm{mmol}, 1.1 \mathrm{eq})$ and $\mathrm{Ph}_{3} \mathrm{GeH}$ (61.0 mg, $\left.0.20 \mathrm{mmol}, 1.0 \mathrm{eq}\right)$. The crude material was purified by flash silica gel chromatography using $\mathrm{Et}_{2} \mathrm{O}$ as the eluent to afford a mixture of $\beta$-vinylgermane $3 \mathbf{u}$ and $\alpha$-vinylgermane $2 \mathbf{u}$ as dark red oil (86.1 $\mathrm{mg}, 97 \%$ isolated yield). The ratio of $\beta / \alpha$ was determined to be $2.5: 1$ by the ${ }^{1} \mathrm{H}$ NMR analysis of the isolated mixture.

${ }^{1} \mathrm{H}$ NMR $\left(400 \mathrm{MHz}, \mathrm{CDCl}_{3}\right) \delta 7.59-7.52(\mathrm{~m}, 6 \mathrm{H}), 7.44-7.37(\mathrm{~m}, 9 \mathrm{H}), 6.27-6.13(\mathrm{~m}, 2 \mathrm{H}$, merged), $2.37-2.23(\mathrm{~m}, 2 \mathrm{H}), 1.53-1.41(\mathrm{~m}, 2 \mathrm{H}), 1.38-1.28(\mathrm{~m}, 10 \mathrm{H}), 0.95-0.90(\mathrm{~m}, 3 \mathrm{H})$.

${ }^{13} \mathrm{C}\left\{{ }^{1} \mathrm{H}\right\}$ NMR $\left(101 \mathrm{MHz}, \mathrm{CDCl}_{3}\right) \delta 151.0,135.3,135.1,128.9,128.2,123.1,36.7,31.9$, 29.5, 29.3, 29.2, 28.7, 22.7, 14.1.

HRMS (EI+) m/z: [M] calcd for $\mathrm{C}_{28} \mathrm{H}_{34} \mathrm{Ge}: 444.1872$; found: 444.1884.

$$
\mathrm{Ph} \sim \mathrm{GePh}_{3}
$$

(E)-triphenyl(styryl)germane (3v). Prepared according to the general procedure using phenylacetylene (24.5 $\mu \mathrm{L}, 0.22 \mathrm{mmol}, 1.1 \mathrm{eq})$ and $\mathrm{Ph}_{3} \mathrm{GeH}$ (61.0 mg, $\left.0.20 \mathrm{mmol}, 1.0 \mathrm{eq}\right)$. The crude material was purified by flash silica gel chromatography using $\mathrm{Et}_{2} \mathrm{O}$ as the eluent to afford a mixture of $\beta$-vinylgermane $\mathbf{3} \mathbf{v}$ and $\alpha$-vinylgermane $\mathbf{2} \mathbf{v}$ as red-brown solid (78.2 $\mathrm{mg}, 96 \%$ isolated yield). The ratio of $\beta / \alpha$ was determined to be $2: 1$ by the ${ }^{1} \mathrm{H}$ NMR analysis of the isolated mixture.

${ }^{1} \mathrm{H}$ NMR $\left(400 \mathrm{MHz}, \mathrm{CDCl}_{3}\right) \delta 7.67-7.59(\mathrm{~m}, 4 \mathrm{H}), 7.57-7.52(\mathrm{~m}, 3 \mathrm{H}), 7.47-7.37(\mathrm{~m}$, $10 \mathrm{H}), 7.34-7.22(\mathrm{~m}, 3 \mathrm{H}), 7.11(\mathrm{~d}, J=18.8 \mathrm{~Hz}, 1 \mathrm{H}), 7.04(\mathrm{~d}, J=18.8 \mathrm{~Hz}, 1 \mathrm{H})$.

${ }^{13} \mathrm{C}\left\{{ }^{1} \mathrm{H}\right\}$ NMR $\left(101 \mathrm{MHz}, \mathrm{CDCl}_{3}\right) \delta 146.9,136.4,135.5,135.2,129.2,129.1,128.6,128.4$, 126.7, 124.1 .

HRMS (El+) m/z: [M] ${ }^{+}$calcd for $\mathrm{C}_{26} \mathrm{H}_{22} \mathrm{Ge:} 408.0933$; found: 408.0948 . 


\section{Upscaled procedure for hydrogermylation of 1-decyne}

In a glovebox, cobalt catalyst $\mathrm{Co}_{2}(\mathrm{CO})_{8}(34.2 \mathrm{mg}, 0.1 \mathrm{mmol}, 0.1 \mathrm{eq})$ was dissolved in $10.0 \mathrm{~mL}$ of DCE (1,2-dichloroethane) in a $20-\mathrm{mL}$ vial with a $0.5 \mathrm{~cm}$ stir bar. The mixture was stirred at room temperature for 10-15 min before 1-decyne $(199.0 \mu \mathrm{L}, 1.1 \mathrm{mmol}, 1.1$ eq) and $\mathrm{Bu}_{3} \mathrm{GeH}(258.0 \mu \mathrm{L}, 1.0 \mathrm{mmol}, 1.0 \mathrm{eq})$ were added via Hamilton glass microsyringes to the reaction mixture. After addition was complete, the reaction was put into a hot plate (that had been pre-heated to $60^{\circ} \mathrm{C}$ ) and stirred for $4 \mathrm{~h}$. Then the vial was taken out of the glovebox, and the reaction mixture was transferred to a $50-\mathrm{mL}$ round bottom flask. The solvent was removed under reduced pressure on a rotary evaporator. Diethyl ether $(5 \mathrm{~mL})$ was added to the residue, and the resulting solution was left overnight in a hume food. On the next day the residue was purified by flash chromatography (using diethyl ether $\mathrm{Et}_{2} \mathrm{O}$ as the eluent); the resulting mixture was heated and stirred on a hot plate at $85^{\circ} \mathrm{C}$ for 2 hours, then filtered through a pad of silica gel (a pipette with about 4 $\mathrm{cm}$ silica gel) for additional purity (with $\mathrm{Et}_{2} \mathrm{O}$ as the eluent).

The filtrate was concentrated under reduced pressure to afford a mixture of $\beta$ vinylgermane $\mathbf{3 a}$ and $\alpha$-vinylgermane $\mathbf{2 a}$ as dark red oil (376.5 mg, $98 \%$ isolated yield). $25 \mathrm{mg}$ of the obtained oil were dissolved in $\mathrm{CDCl}_{3}$ for ${ }^{1} \mathrm{H}$ NMR analysis. Observed chemical shift values matched the values reported in the previous section. The ratio of $\beta / \alpha$ was determined to be $11.2: 1$ by the ${ }^{1} \mathrm{H}$ NMR analysis of the isolated mixture. 


\section{Mechanistic studies}

Table S6. Mechanistic investigation

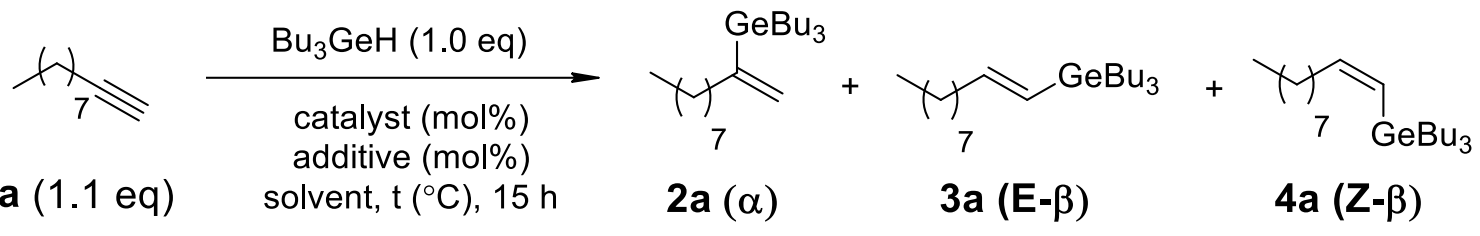

\begin{tabular}{ccccccc}
\hline Entry & Catalytic system & $\mathrm{T}\left({ }^{\circ} \mathrm{C}\right)$ & Solvent & 2a (\%) & 3a (\%) & 4a (\%) \\
\hline 1 & $\mathrm{NaCo}(\mathrm{CO})_{4}(10 \%)+\mathrm{PhCOOH}(10 \%)$ & 85 & $\mathrm{DCE}$ & 9 & 82 & 0 \\
2 & $\mathrm{NaCo}(\mathrm{CO})_{4}(10 \%)+\mathrm{tBuONa}(10 \%)$ & 100 & toluene & 5 & 3 & 2 \\
3 & $\mathrm{Co}_{2}(\mathrm{CO})_{8}(10 \%)+\mathrm{PhCOOH}(10 \%)$ & 60 & $\mathrm{DCE}$ & 9 & 91 & 0 \\
4 & $\mathrm{Ph}_{3} \mathrm{Si}-\mathrm{Co}(\mathrm{CO})_{4}(10 \%)$ & 85 & $\mathrm{DME}$ & 11 & 88 & 0 \\
5 & $\mathrm{Ph}_{3} \mathrm{Si}-\mathrm{Co}(\mathrm{CO})_{4}(10 \%)+\mathrm{MeOH}(10 \%)$ & 85 & $\mathrm{DME}$ & 10 & 87 & 0 \\
6 & $\mathrm{Ph}_{3} \mathrm{Si}-\mathrm{Co}(\mathrm{CO})_{4}(10 \%)$ & 100 & toluene & 15 & 68 & 2 \\
7 & $\mathrm{Ph}_{3} \mathrm{Si}-\mathrm{Co}(\mathrm{CO})_{4}(10 \%)+\mathrm{MeOH}(10 \%)$ & 100 & toluene & 8 & 66 & 1 \\
8 & $\mathrm{Co}(\mathrm{TPP})^{a}(10 \%)$ & 100 & toluene & 0 & 0 & 0 \\
9 & $\mathrm{Co}(\mathrm{Pc})^{b}(10 \%)$ & 100 & toluene & 0 & 0 & 0 \\
\hline
\end{tabular}

${ }^{a}$ Cobalt(II) tetraphenylporphyrin; ${ }^{b}$ Cobalt(II) phthalocyanine. 
6. ${ }^{1} \mathrm{H},{ }^{13} \mathrm{C}\left\{{ }^{1} \mathrm{H}\right\}$ NMR spectra

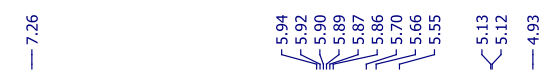

$\mathrm{CDCl}_{3}, 400 \mathrm{MHz}$

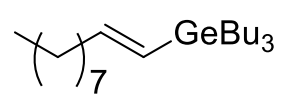

3a $(\beta / \alpha=9.5: 1)$
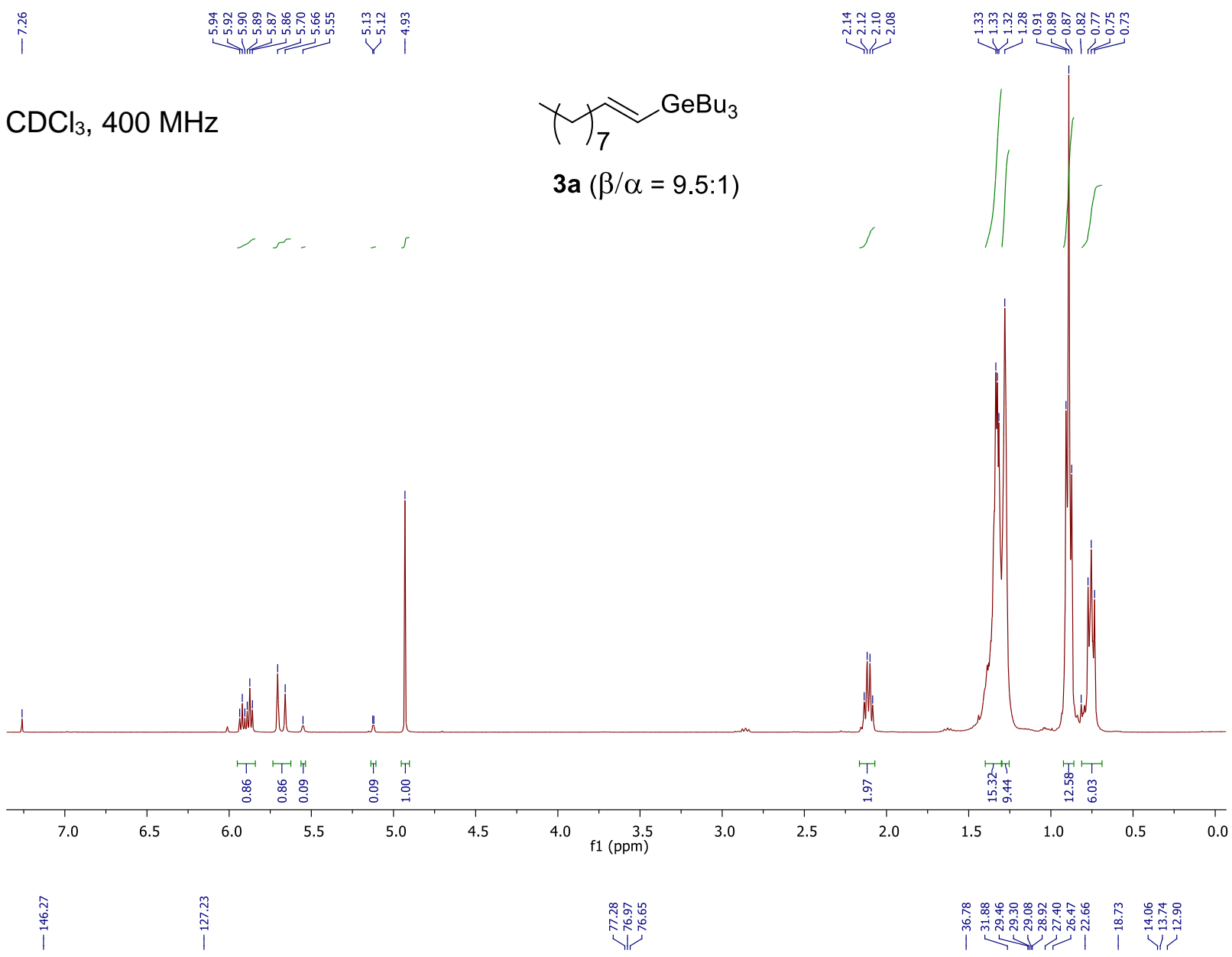

$\mathrm{CDCl}_{3}, 101 \mathrm{MHz}$

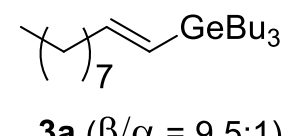

3a $(\beta / \alpha=9.5: 1)$

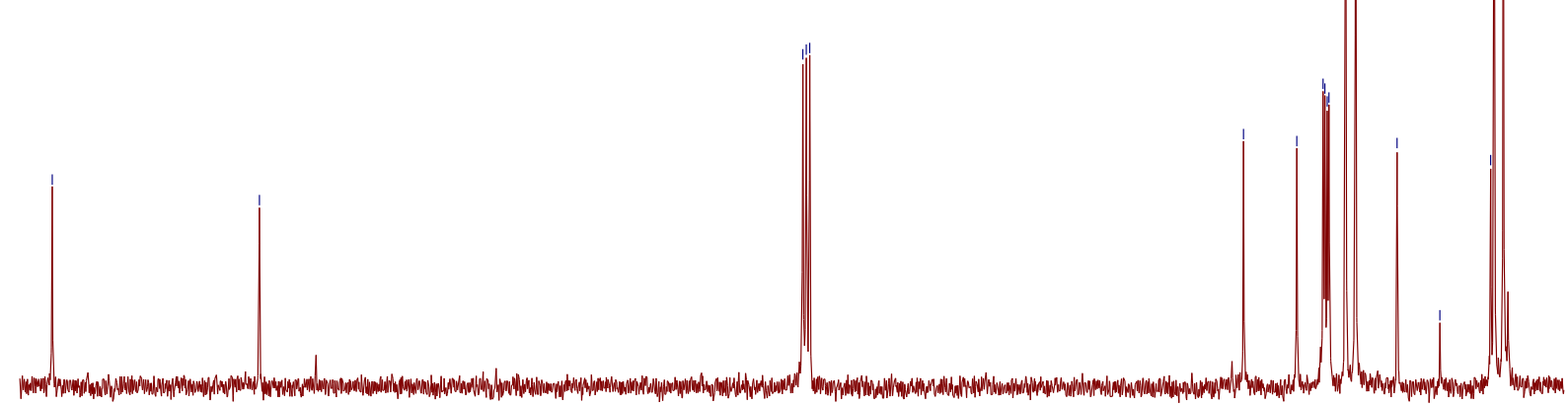

$\begin{array}{lllllllllllllllllllllllllllllllllll}145 & 140 & 135 & 130 & 125 & 120 & 115 & 110 & 105 & 100 & 95 & 90 & 85 & 80 & 75 & 70 & 65 & 60 & 55 & 50 & 45 & 40 & 35 & 30 & 25 & 20 & 15 & 10\end{array}$ 


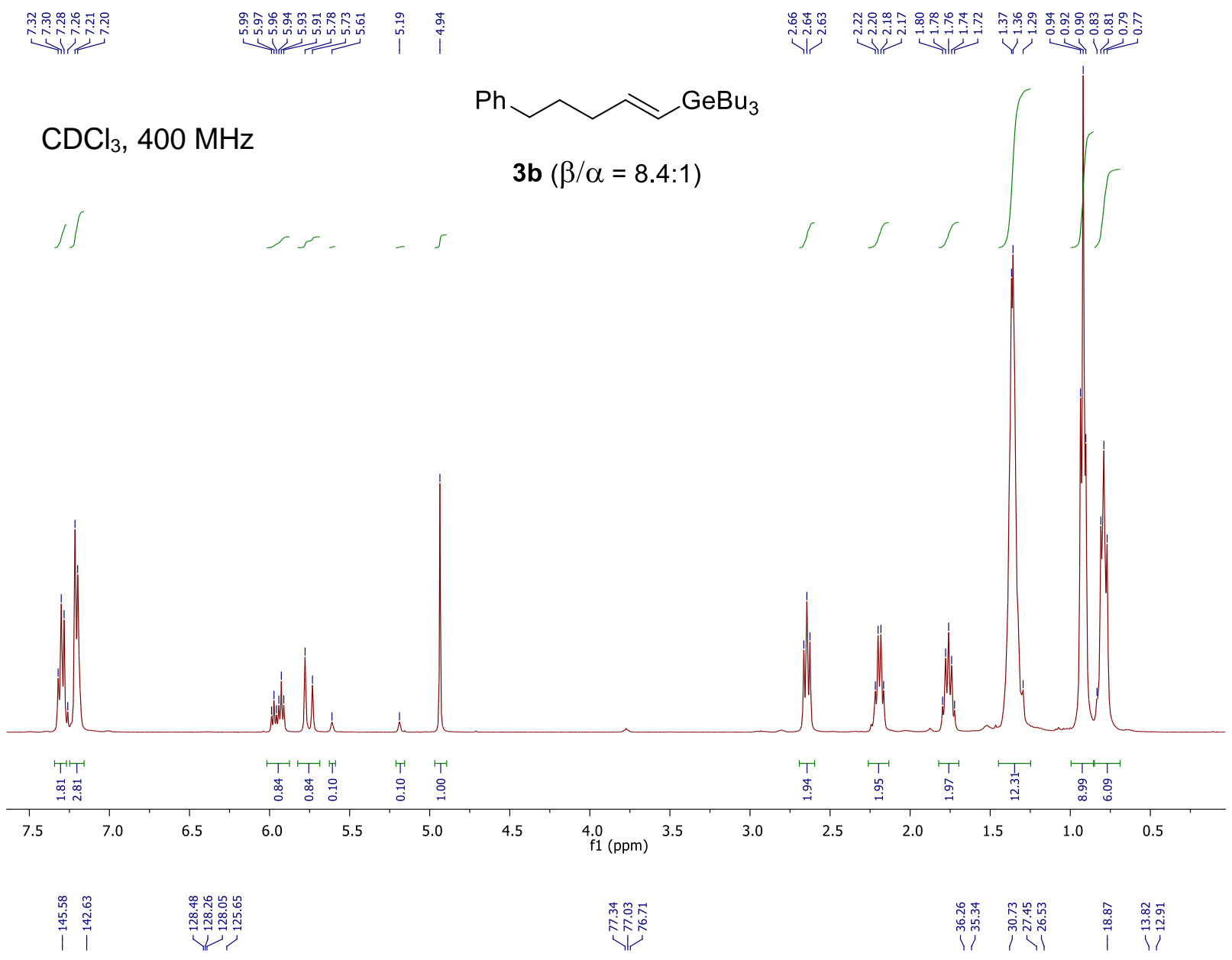

$\mathrm{CDCl}_{3}, 101 \mathrm{MHz}$
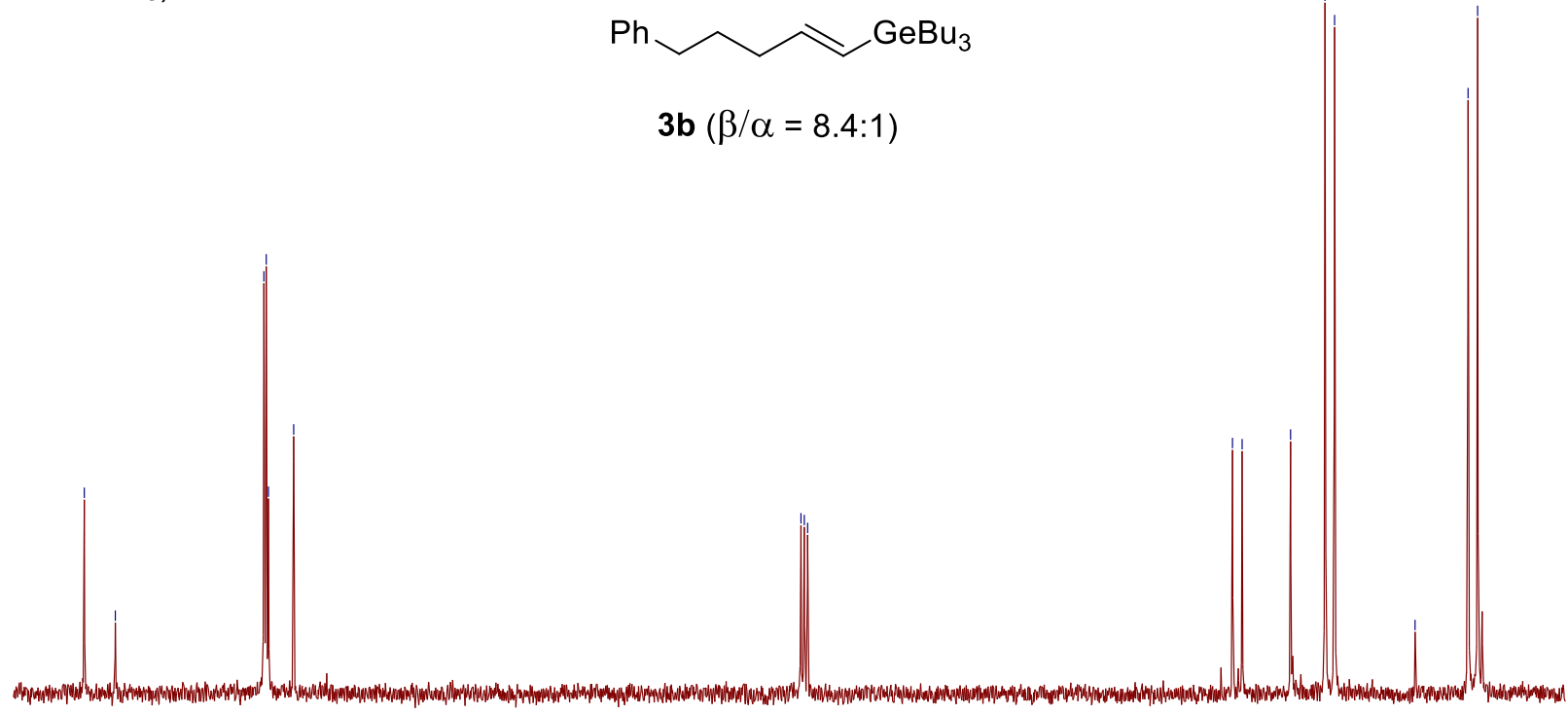

$3 b(\beta / \alpha=8.4: 1)$

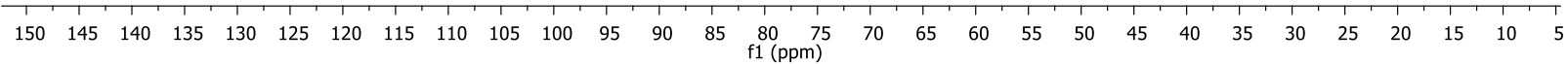



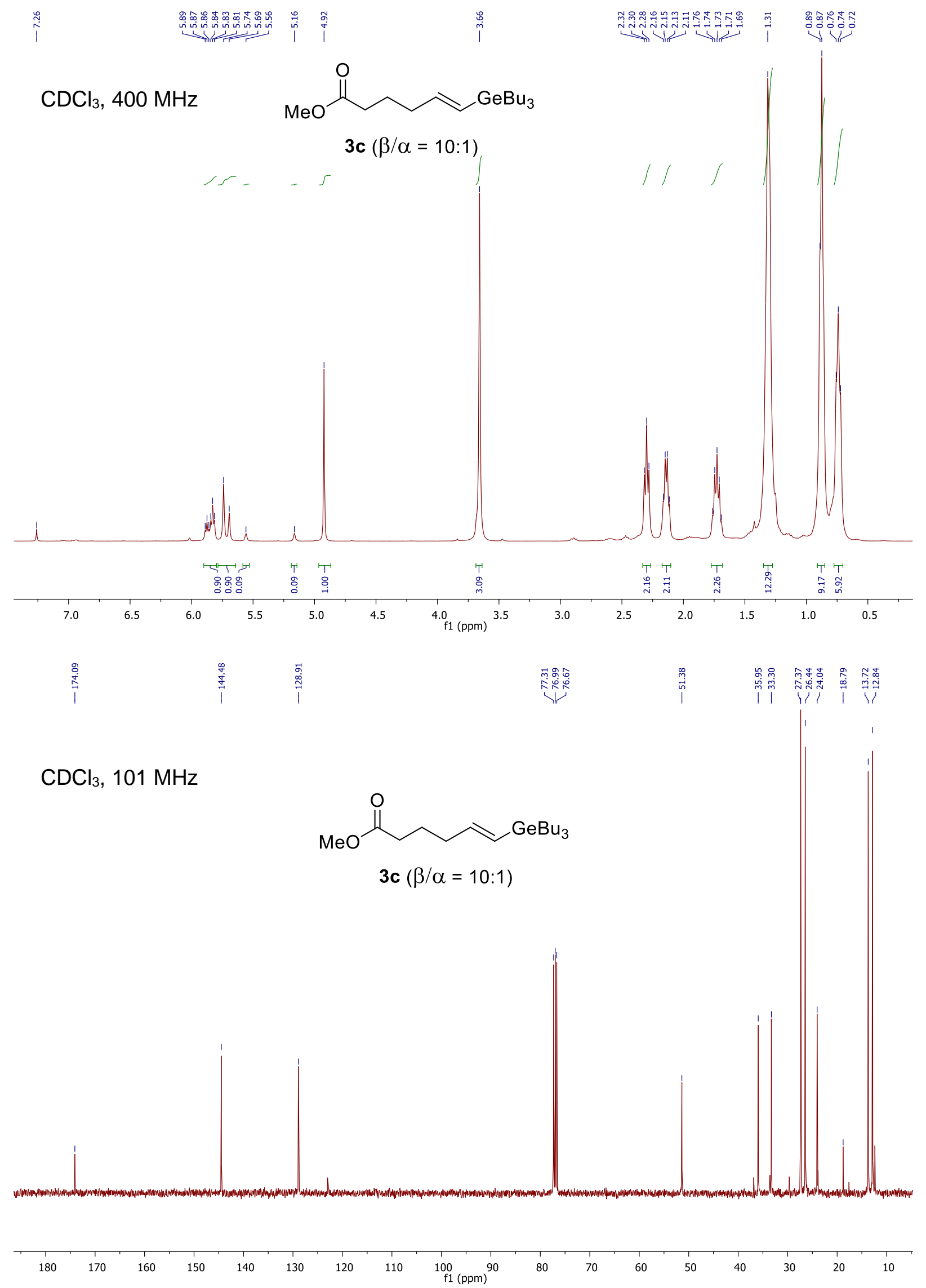


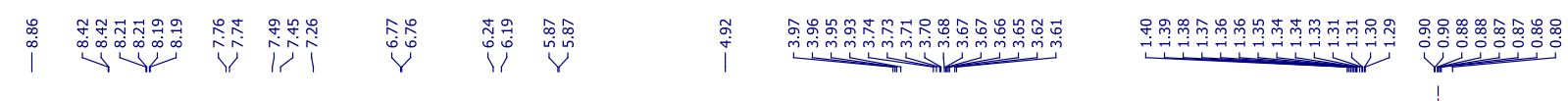

$\mathrm{CDCl}_{3}, 400 \mathrm{MHz}$

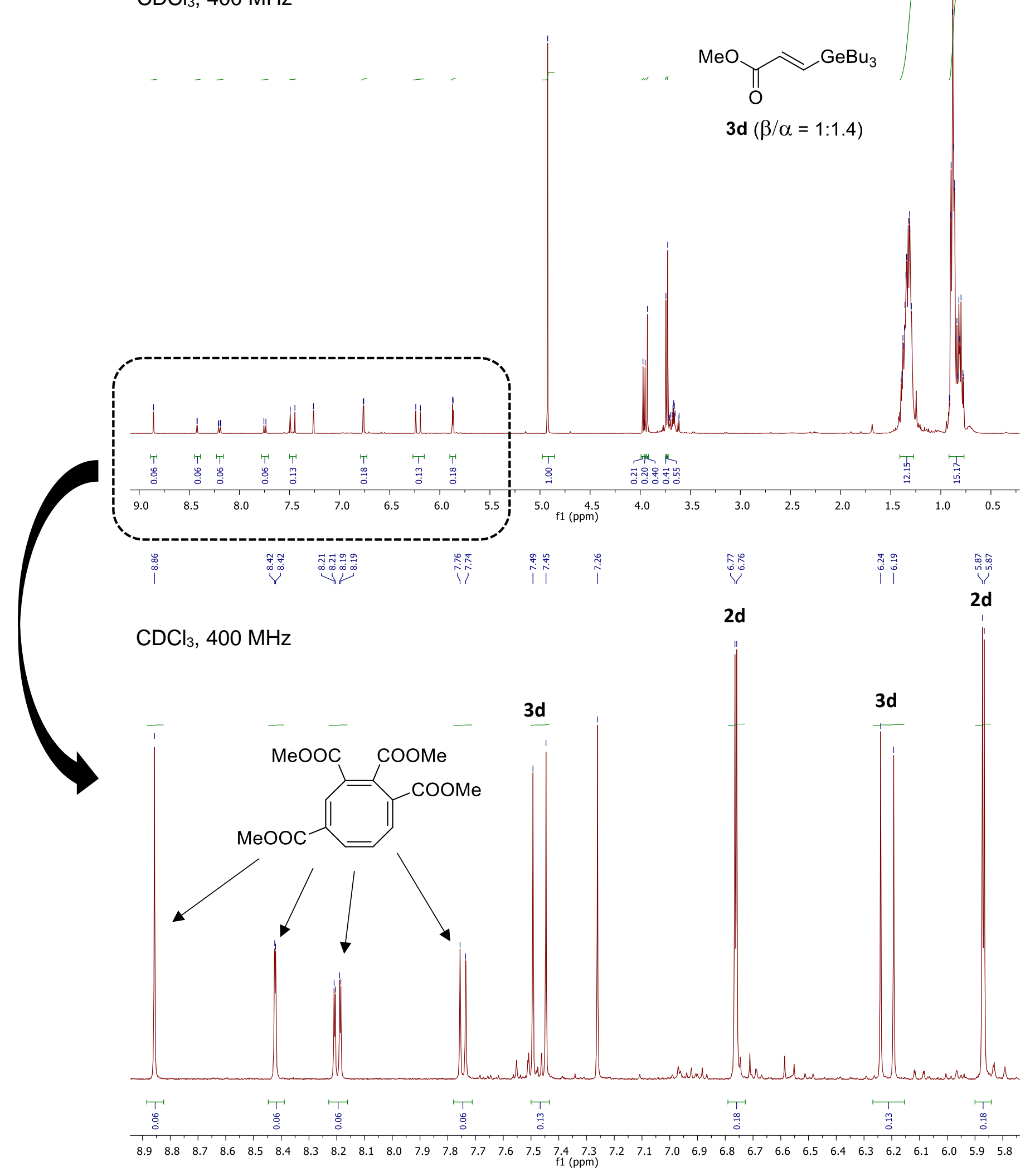




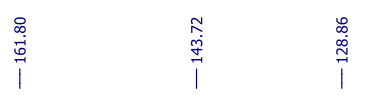

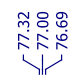

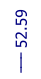

\/

$\mathrm{CDCl}_{3}, 101 \mathrm{MHz}$<smiles>COC(=O)/C=C/[GeH2]C(C)(C)C</smiles>

3d $(\beta / \alpha=1: 1.4)$

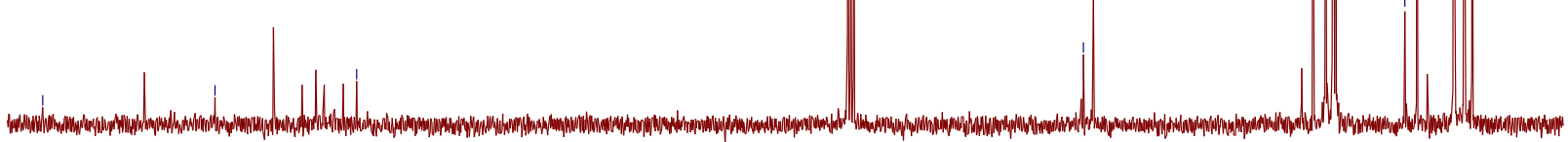

\begin{tabular}{|c|c|c|c|c|c|c|c|c|c|c|c|c|c|c|}
\hline 160 & 150 & 140 & 130 & 120 & 110 & 100 & $90 \underset{\mathrm{f} 1(\mathrm{ppm})}{80}$ & 70 & 60 & 50 & 40 & 30 & 20 & 10 \\
\hline لـ & 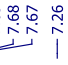 & & & 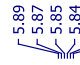 & 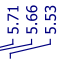 & 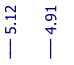 & & & & & & & & \\
\hline
\end{tabular}

$\mathrm{CDCl}_{3}, 400 \mathrm{MHz}$

$\iint$<smiles>CC(C)(C)OC(C)(C)C=CCCCCN1C(=O)c2ccccc2C1=O</smiles>

3e $(\beta / \alpha=11: 1)$

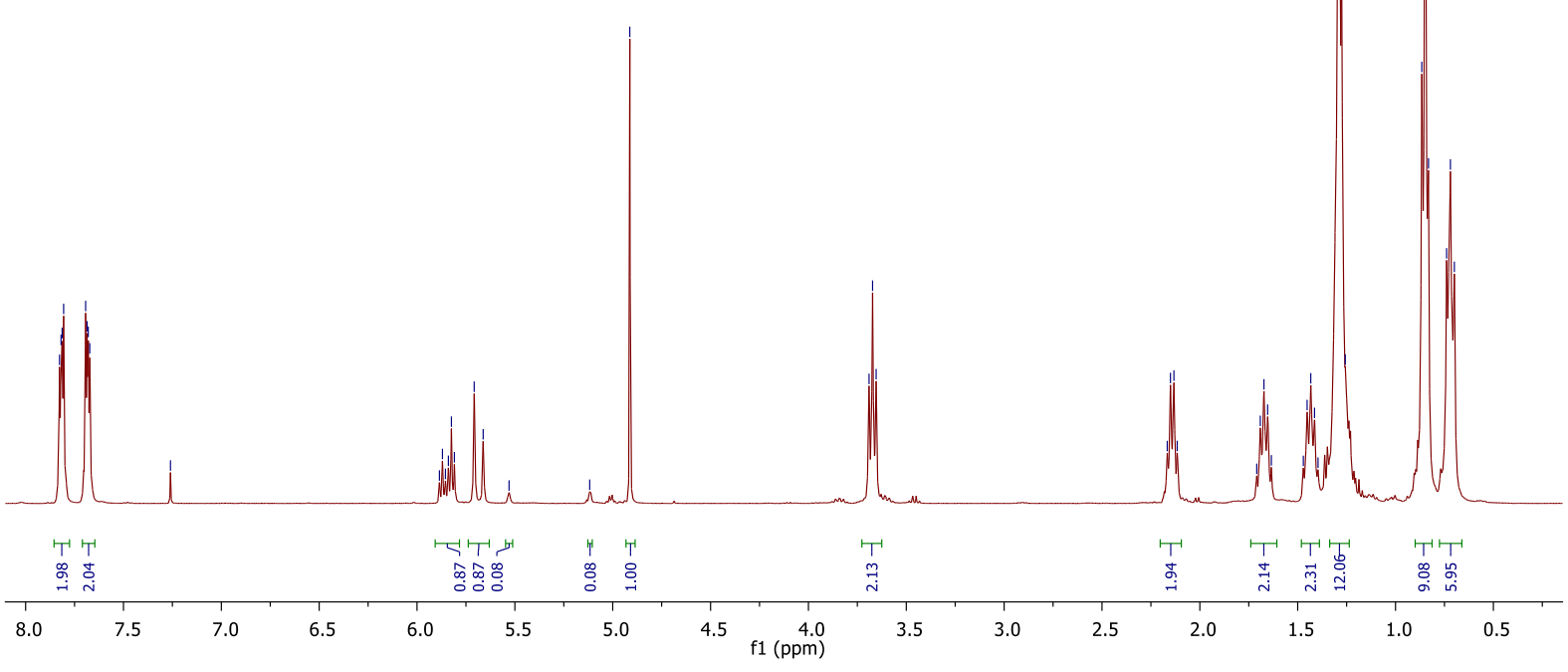

S-24 

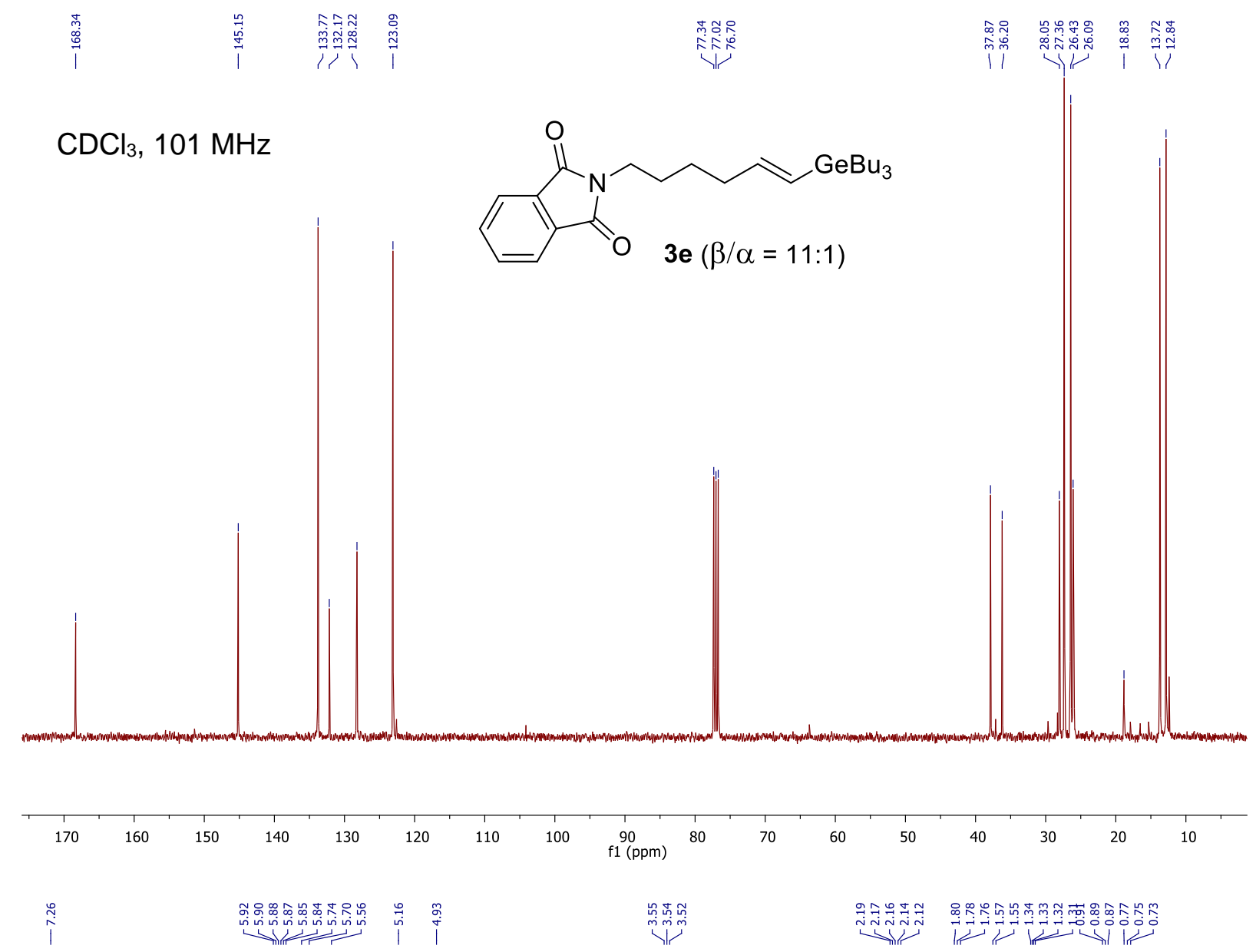

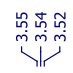

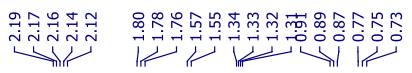

$\mathrm{CDCl}_{3}, 400 \mathrm{MHz}$
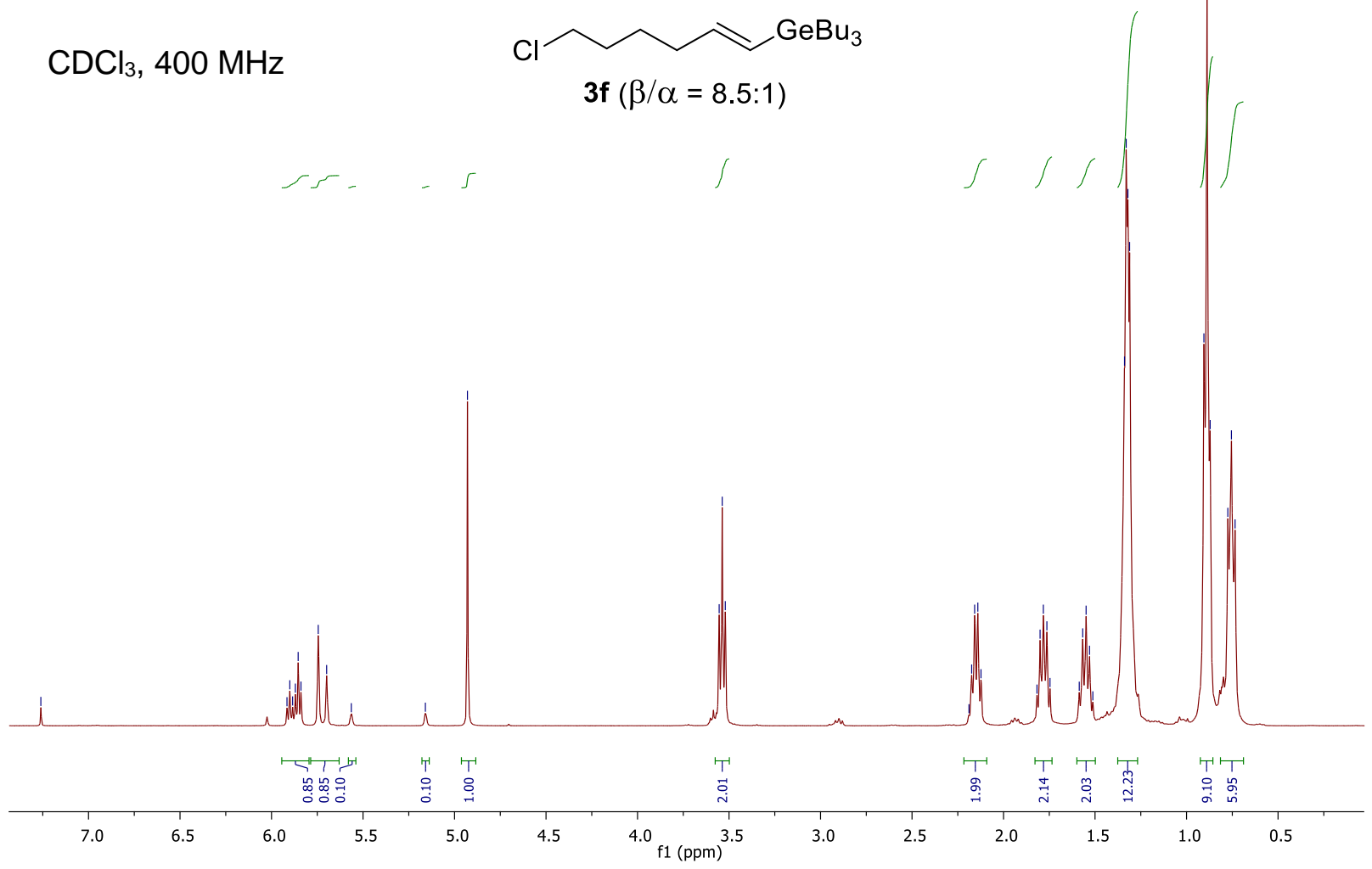


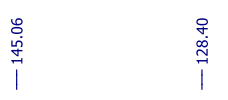

$\mathrm{CDCl}_{3}, 101 \mathrm{MHz}$

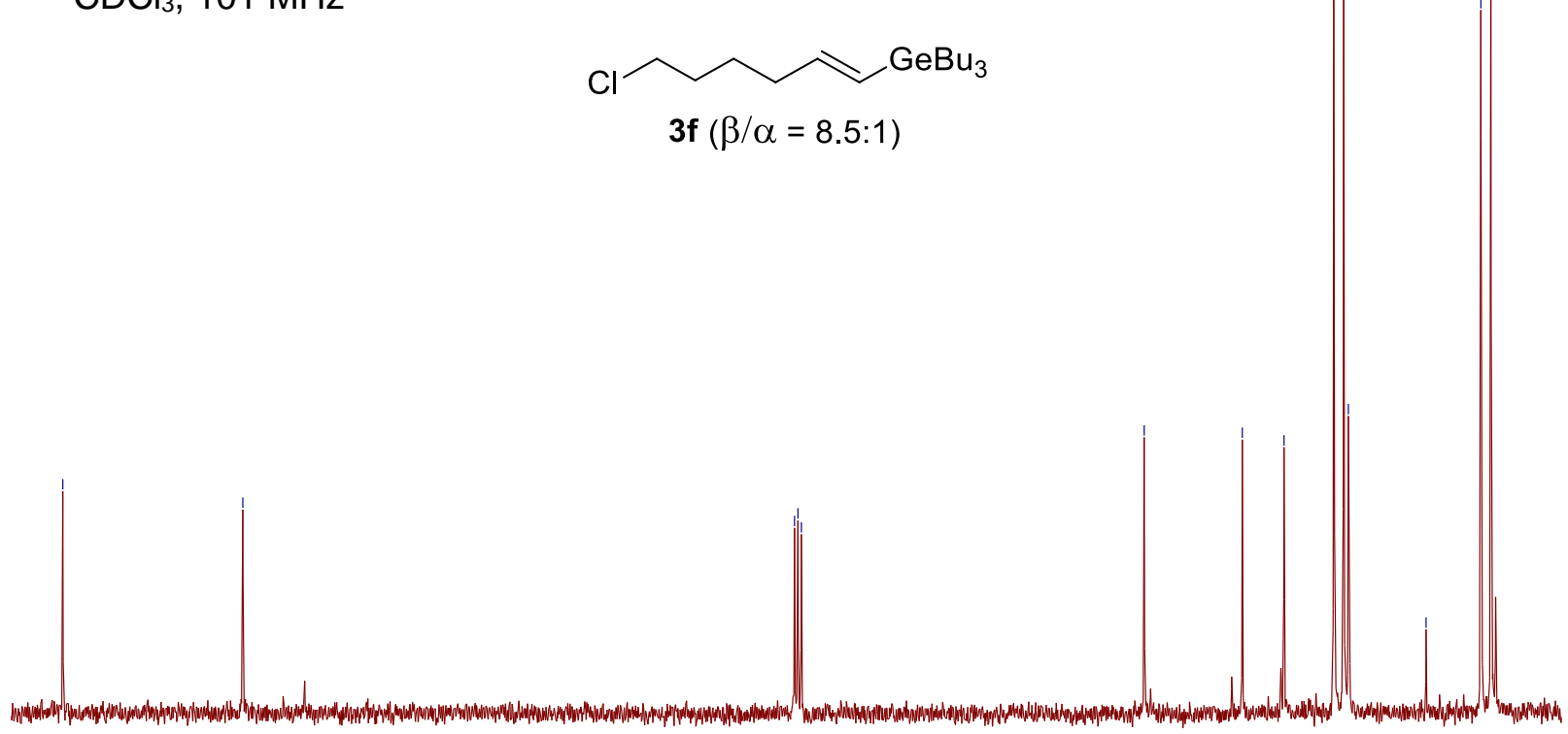

$\begin{array}{llllllllllllllllllllllllllllllllllll}145 & 140 & 135 & 130 & 125 & 120 & 115 & 110 & 105 & 100 & 95 & 90 & 85 & \begin{array}{c}80 \\ \mathrm{f}(\mathrm{ppm})\end{array} & 70 & 65 & 60 & 55 & 50 & 45 & 40 & 35 & 30 & 25 & 20 & 15 & 10\end{array}$

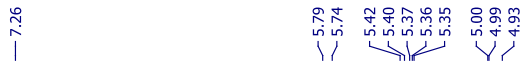

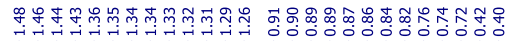

$\mathrm{CDCl}_{3}, 400 \mathrm{MHz}$

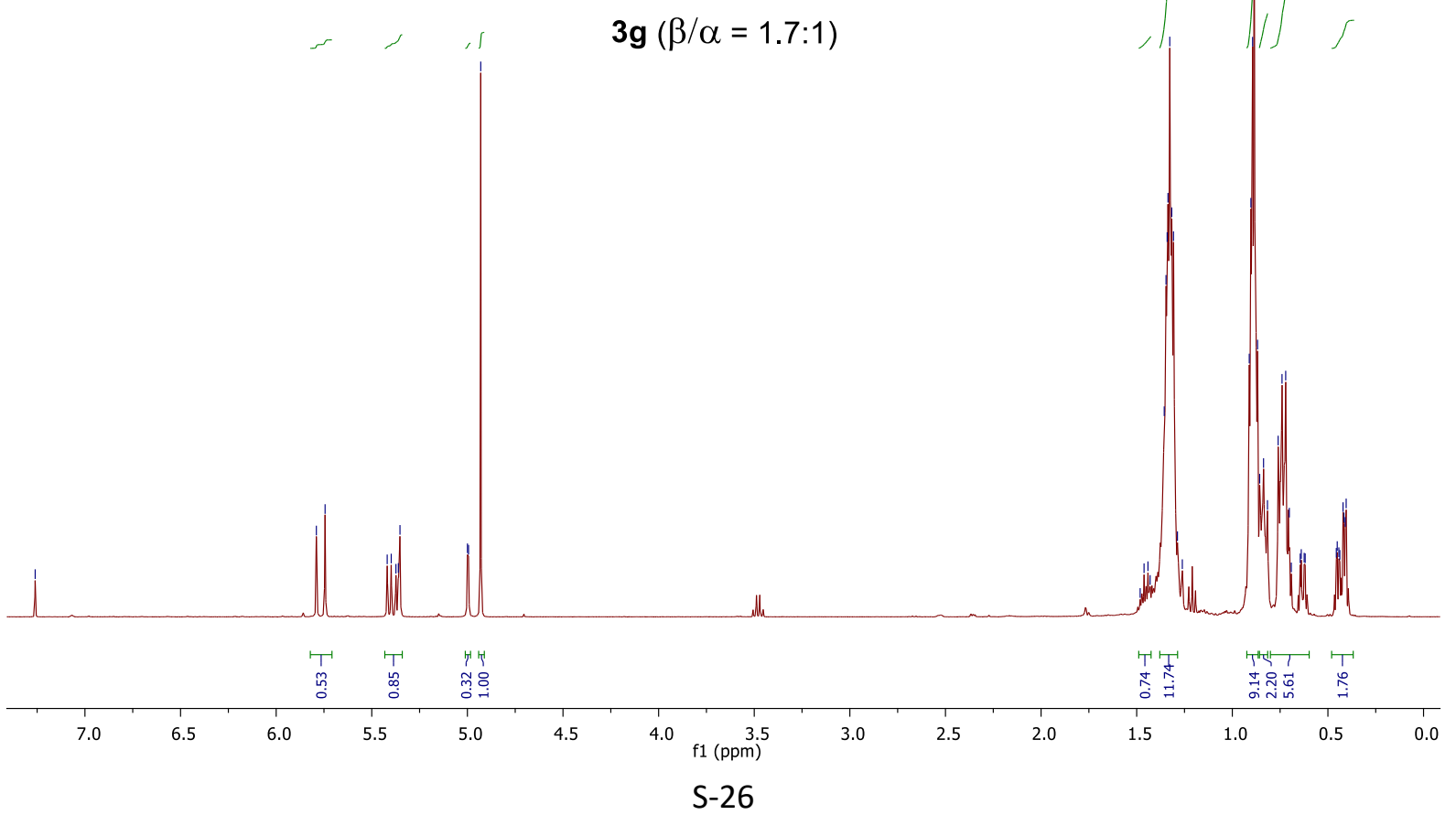




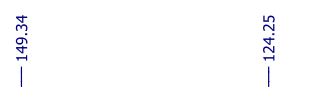

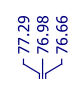

$\mathrm{CDCl}_{3}, 101 \mathrm{MHz}$

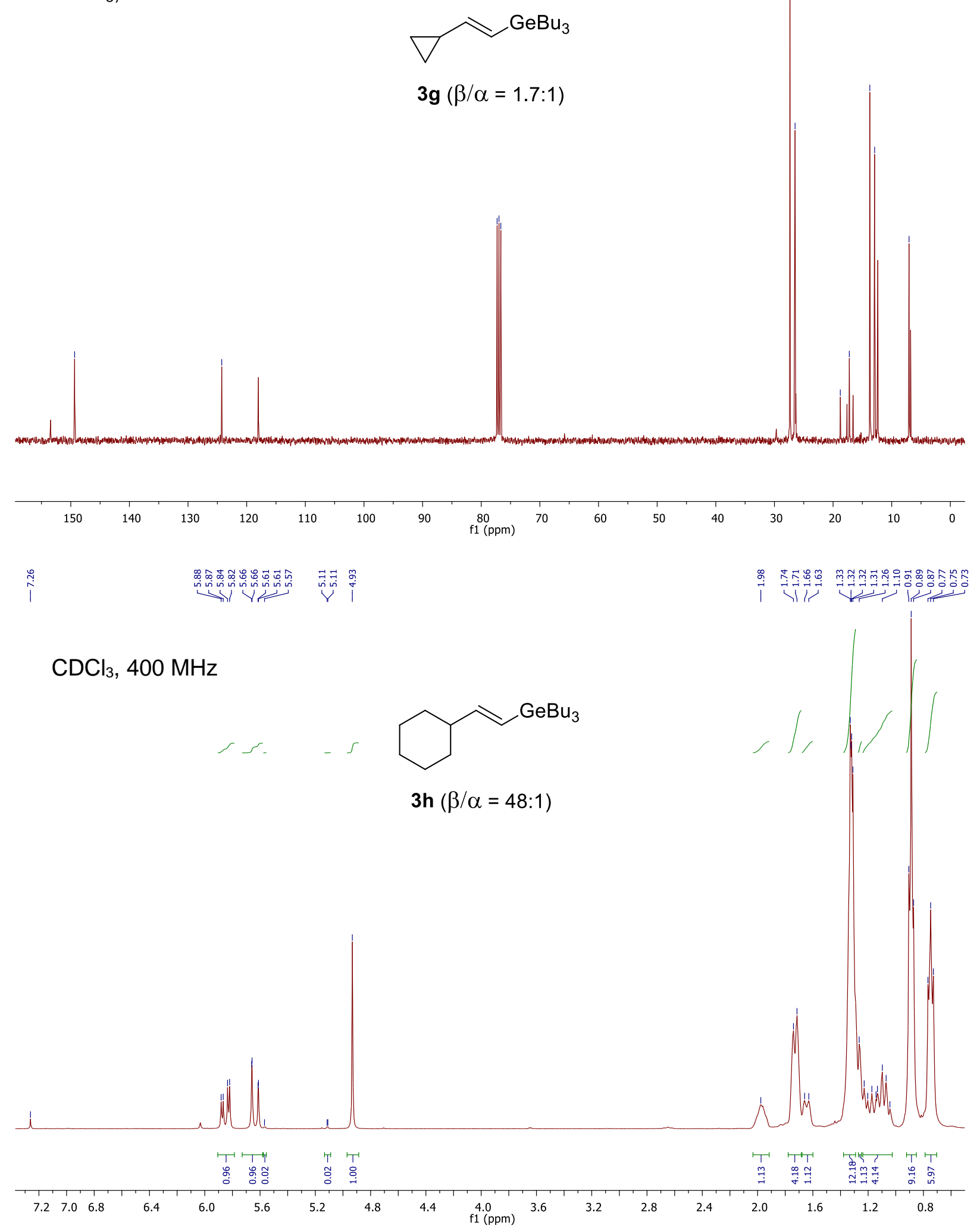



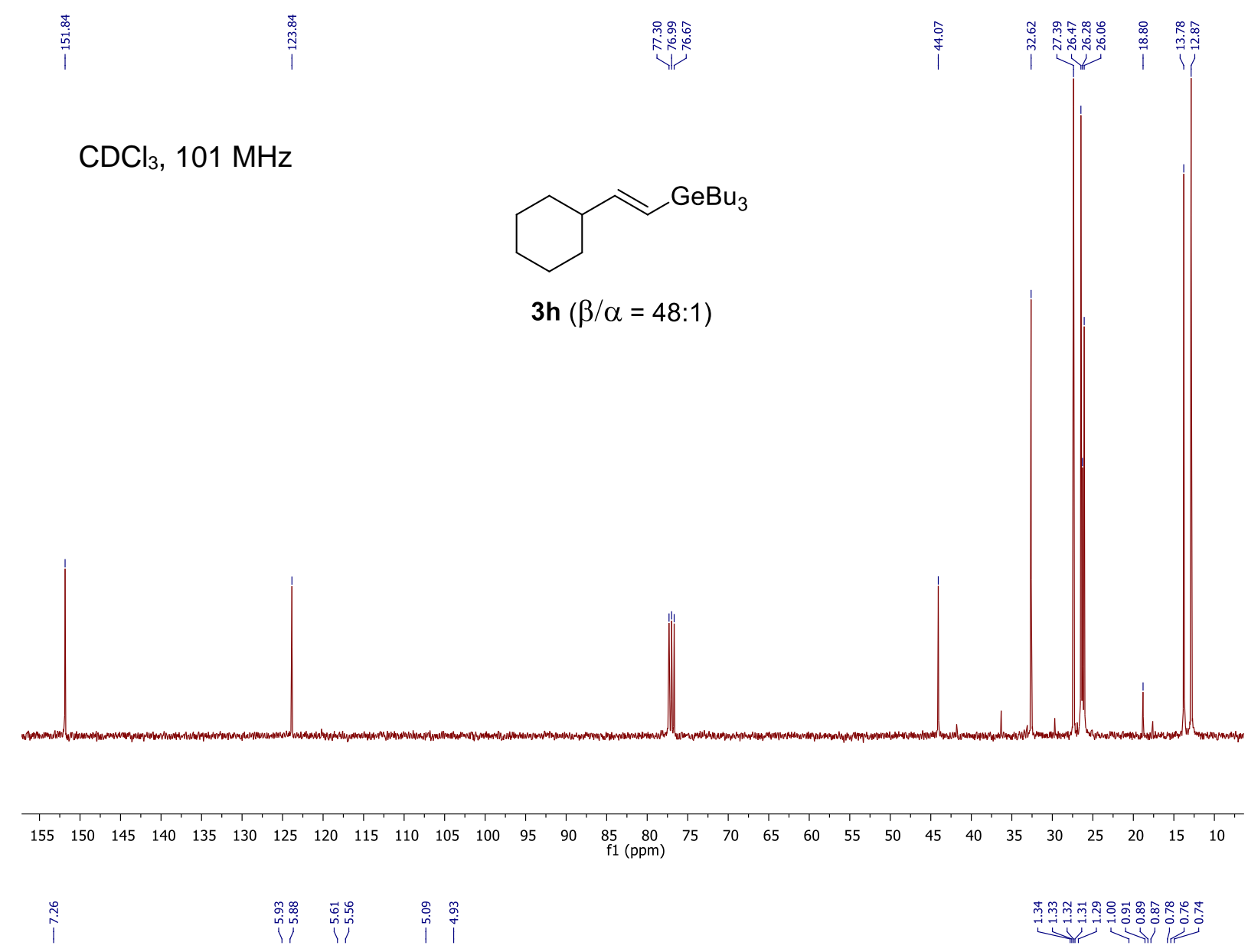

$\mathrm{CDCl}_{3}, 400 \mathrm{MHz}$

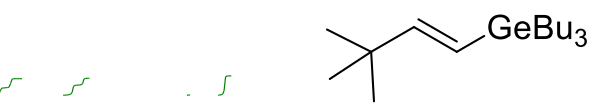

$$
3 \mathbf{i}(\beta / \alpha=43: 1)
$$
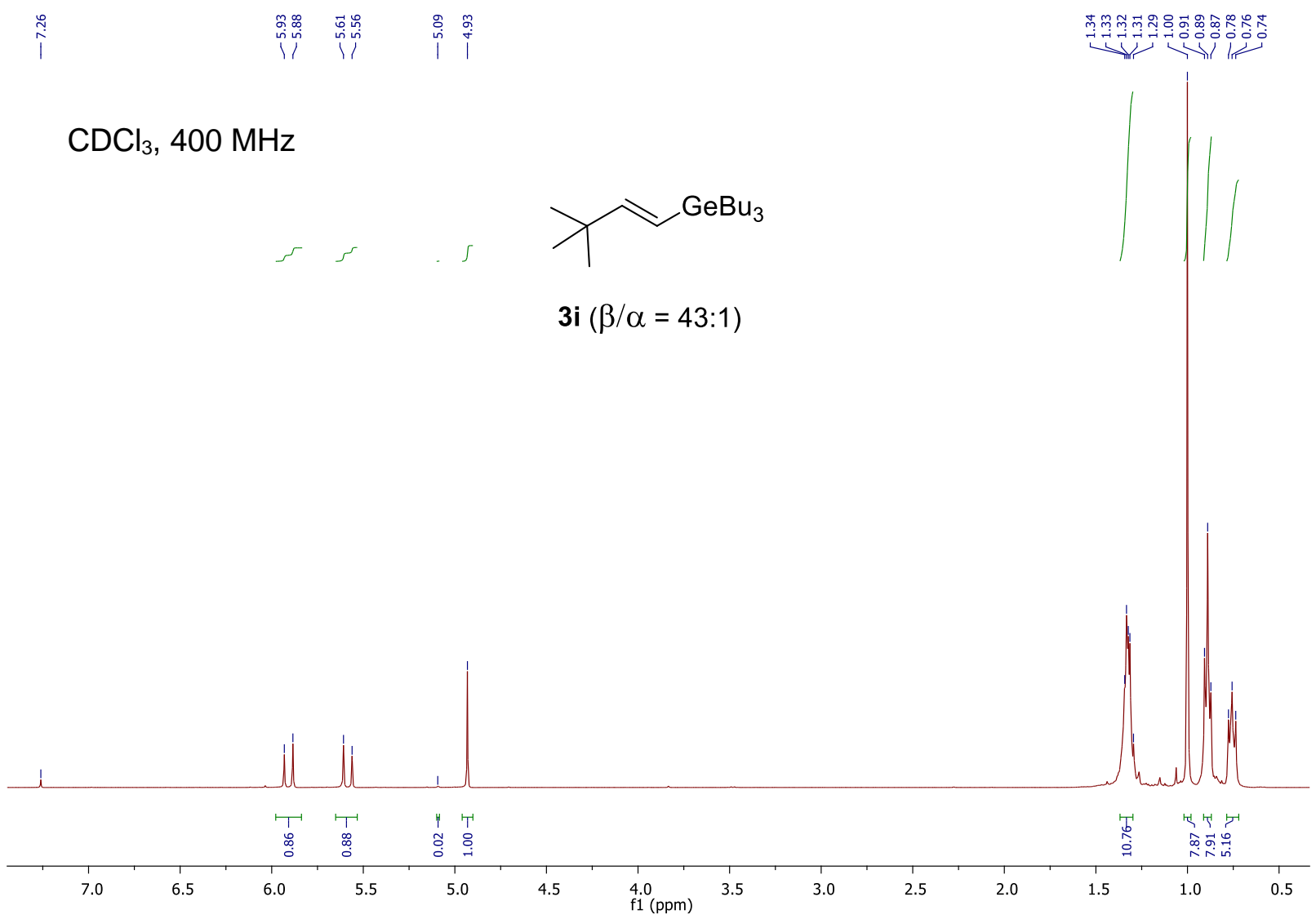

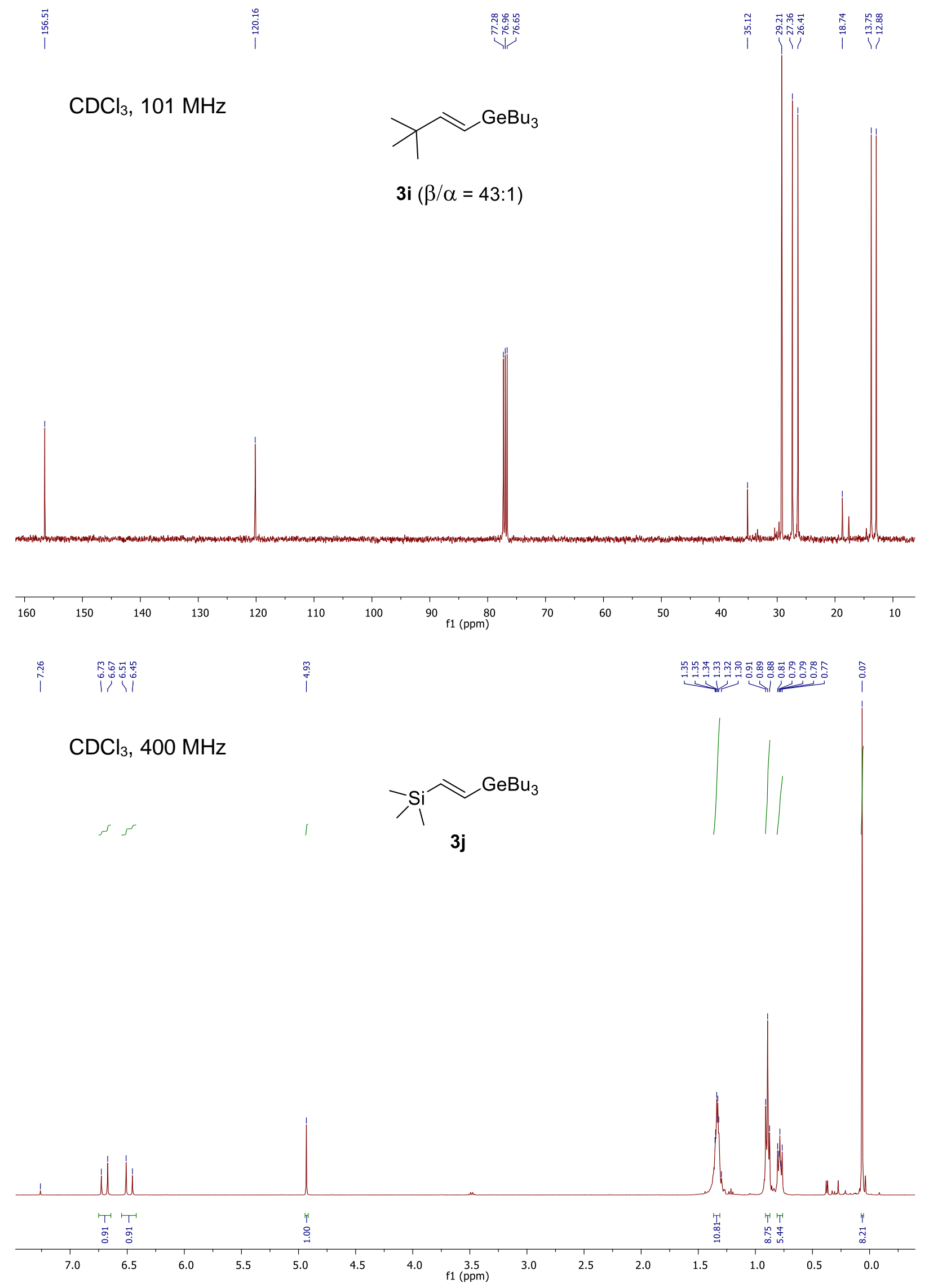

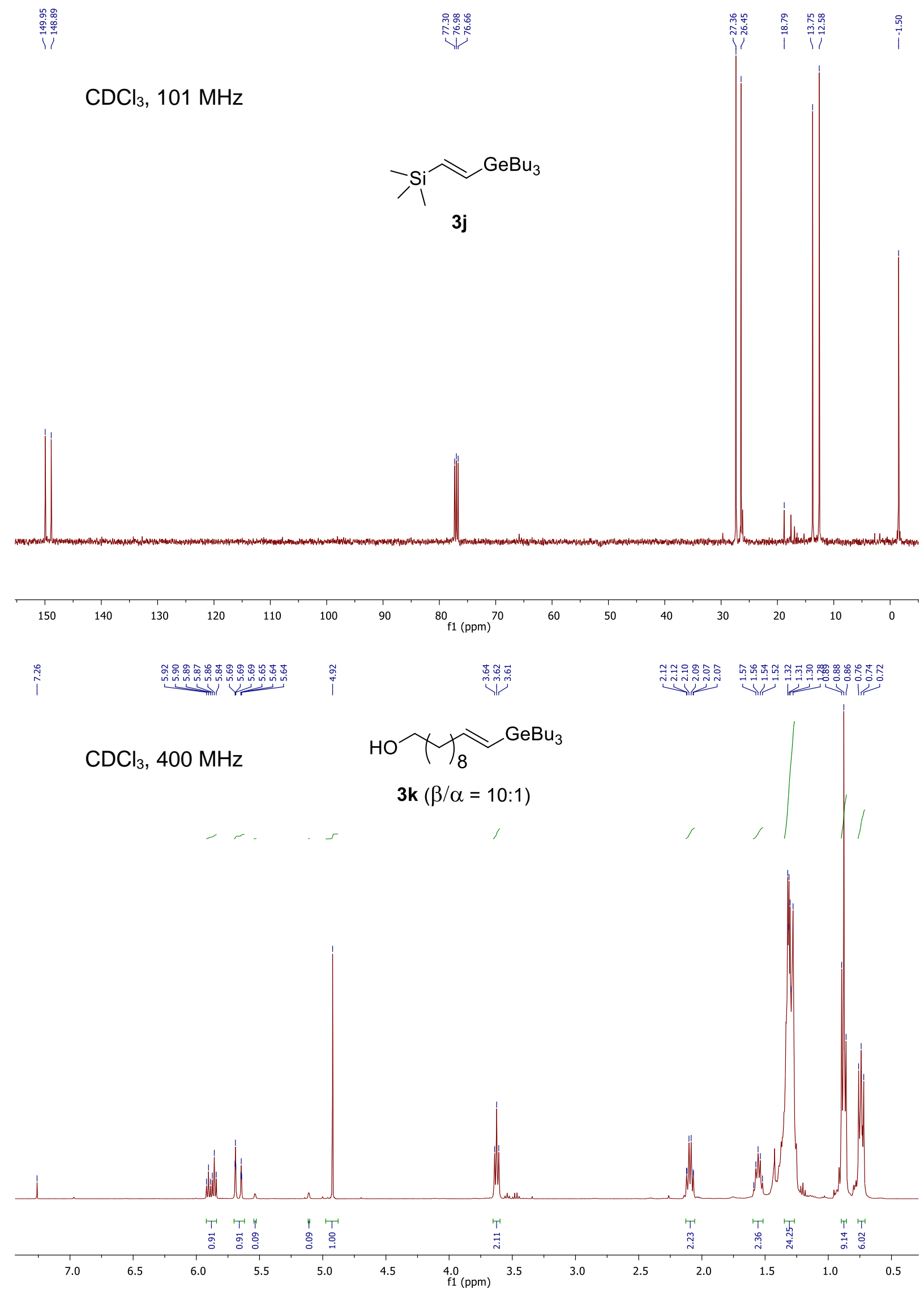

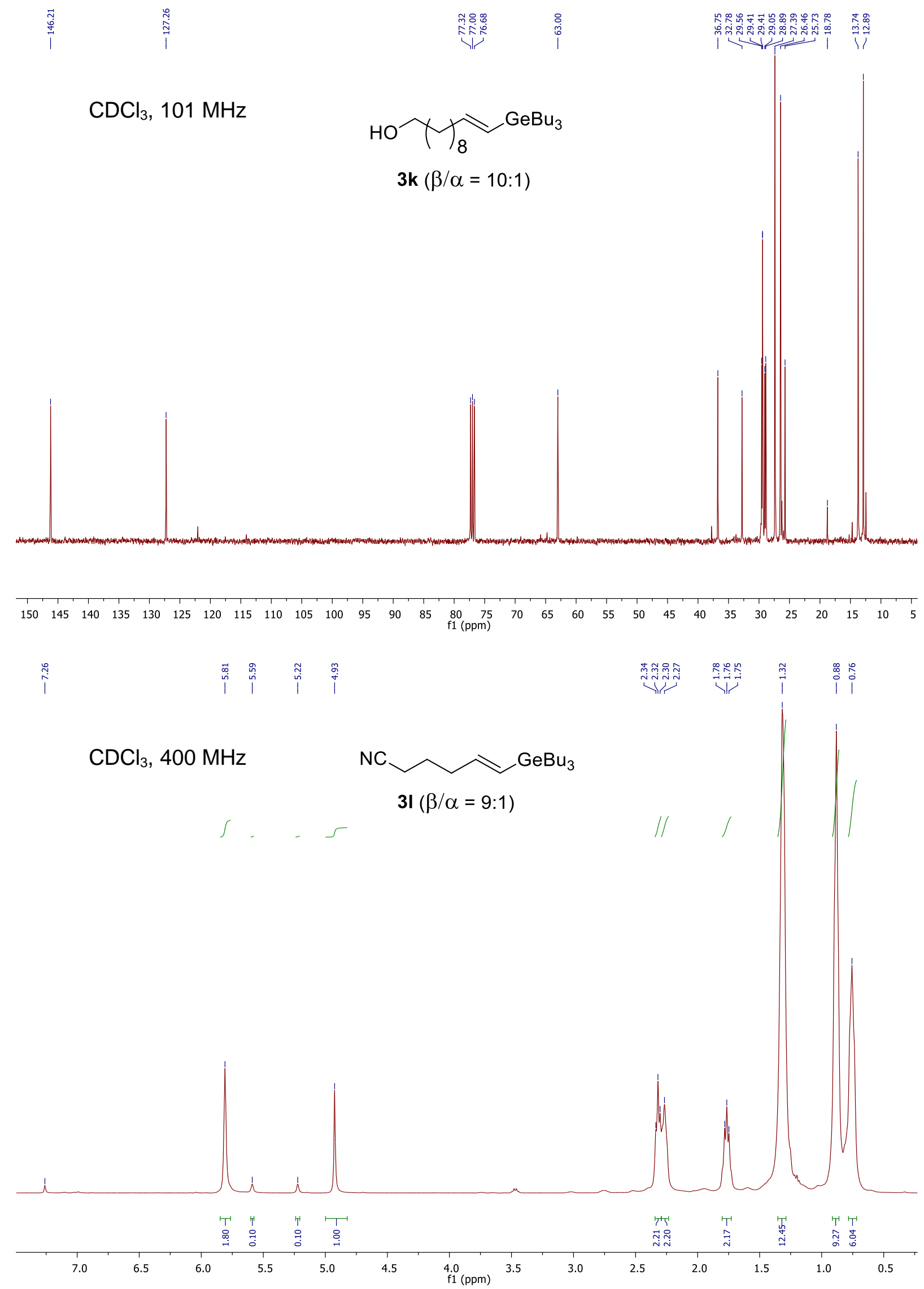


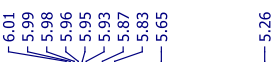

Acetone-d6, $400 \mathrm{MHz}$

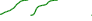

$\mathrm{NC}$

3I $(\beta / \alpha=9: 1)$

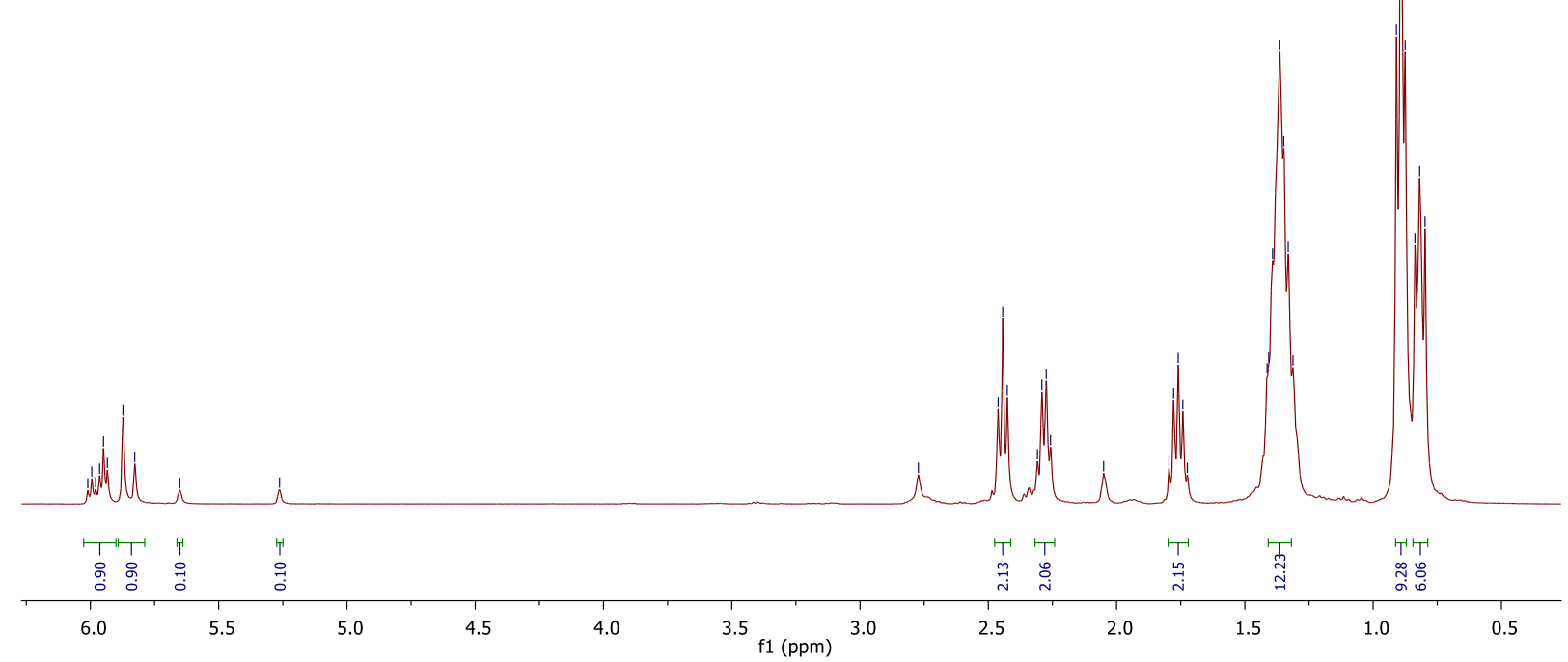

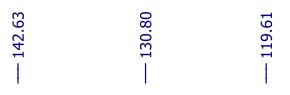

$\mathrm{CDCl}_{3}, 101 \mathrm{MHz}$

$\mathrm{NC} \sim \mathrm{GeBu}_{3}$

3I $(\beta / \alpha=9: 1)$

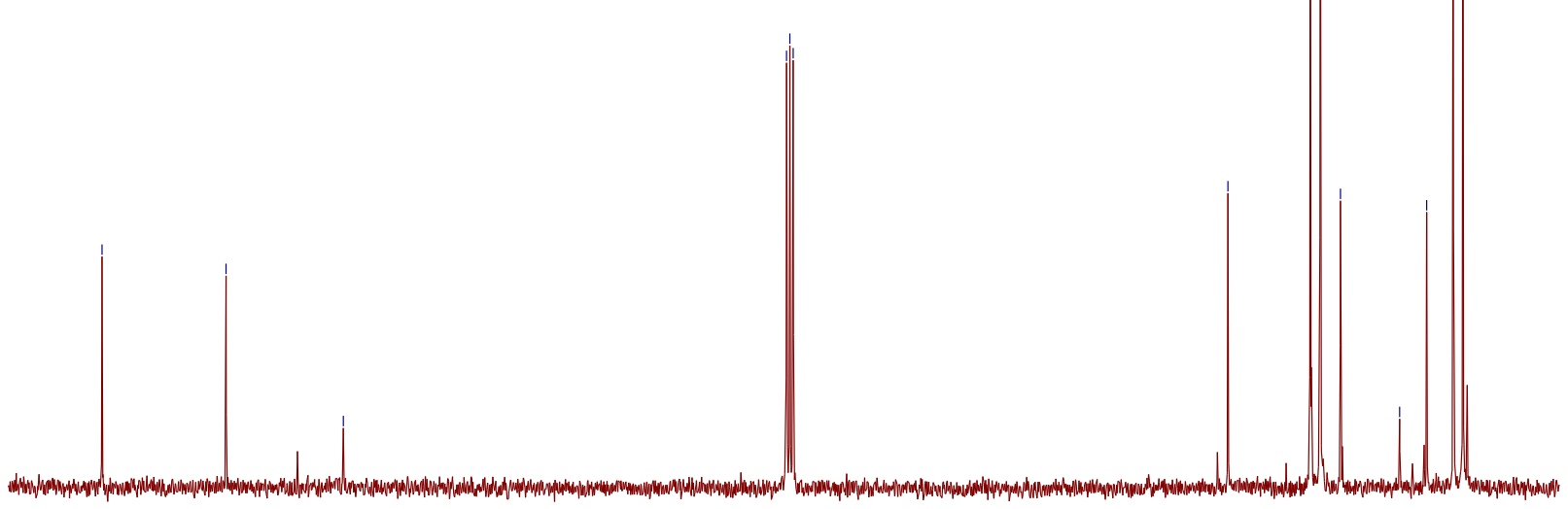

$\begin{array}{lllllllllllllllllllllllllllllllllllllllll}150 & 145 & 140 & 135 & 130 & 125 & 120 & 115 & 110 & 105 & 100 & 95 & 90 & 85 & 80 & 75 & 70 & 65 & 60 & 55 & 50 & 45 & 40 & 35 & 30 & 25 & 20 & 15 & 10 & 5\end{array}$ 


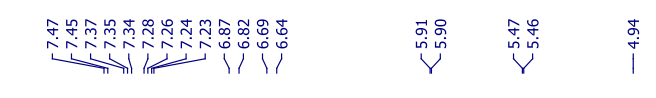

$\mathrm{CDCl}_{3}, 400 \mathrm{MHz}$

T2
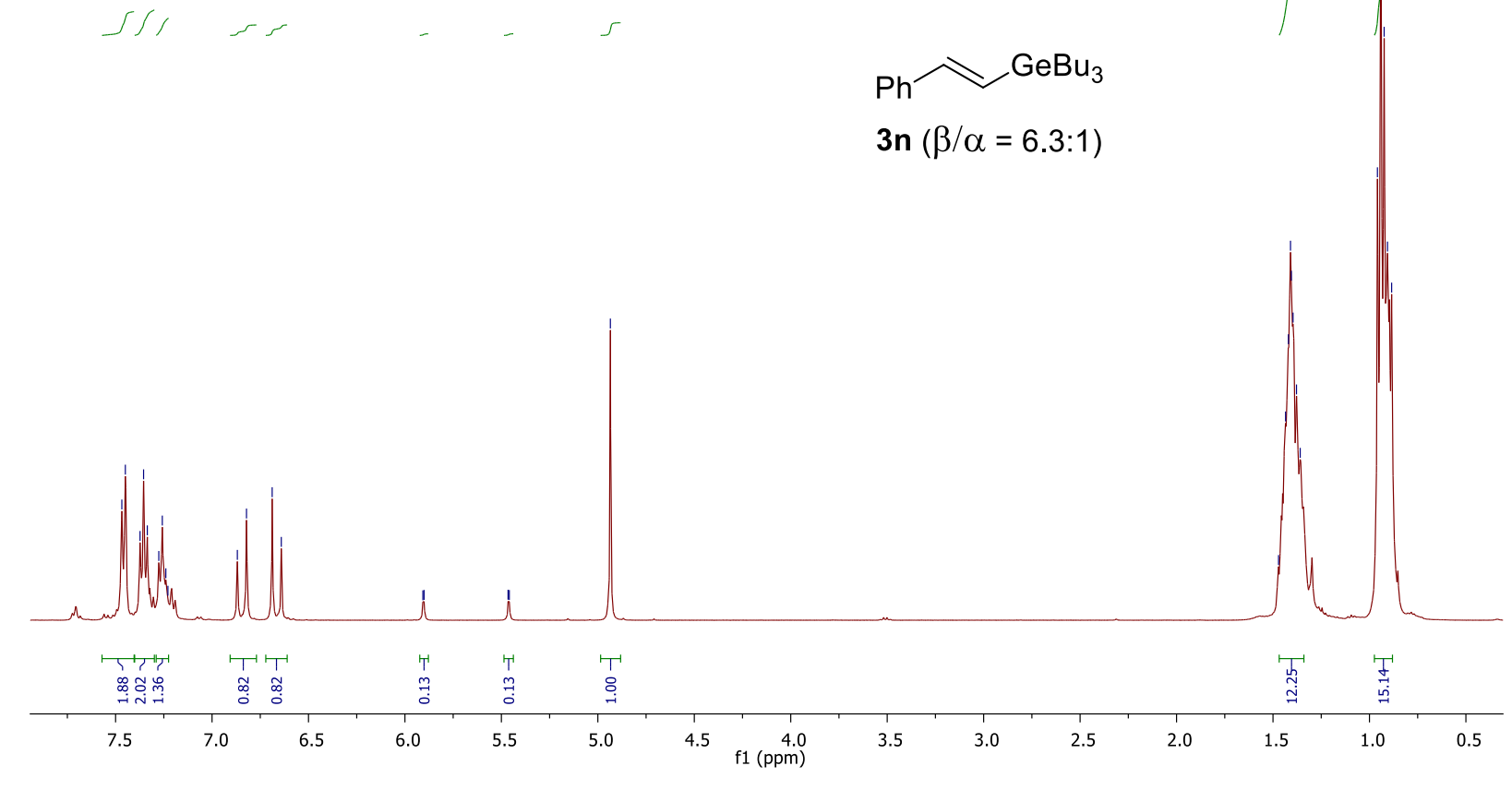

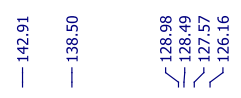

$\mathrm{CDCl}_{3}, 101 \mathrm{MHz}$
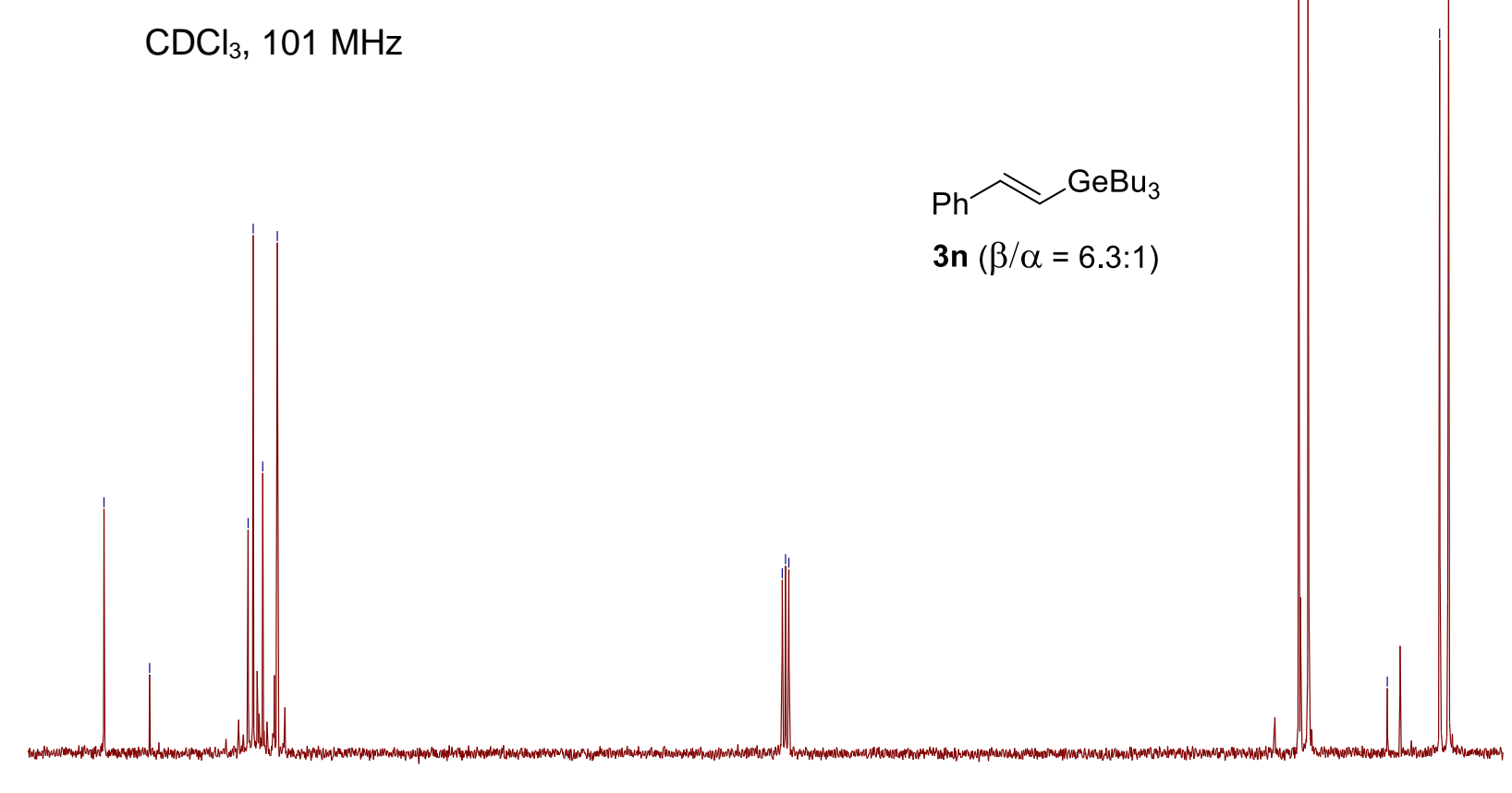

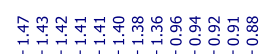

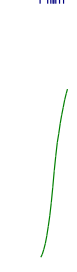

3n $(\beta / \alpha=6.3: 1)$

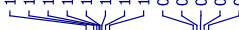

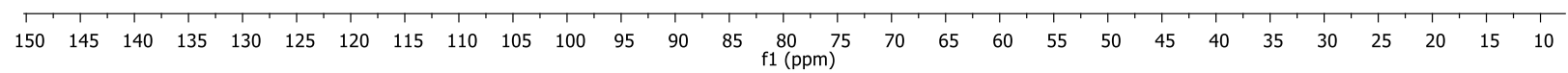




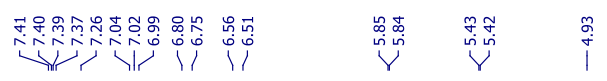

$\mathrm{CDCl}_{3}, 400 \mathrm{MHz}$

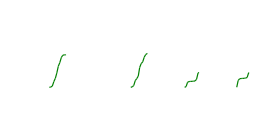<smiles>CC(C)(C)O[13C]=Cc1ccc(F)cc1</smiles>

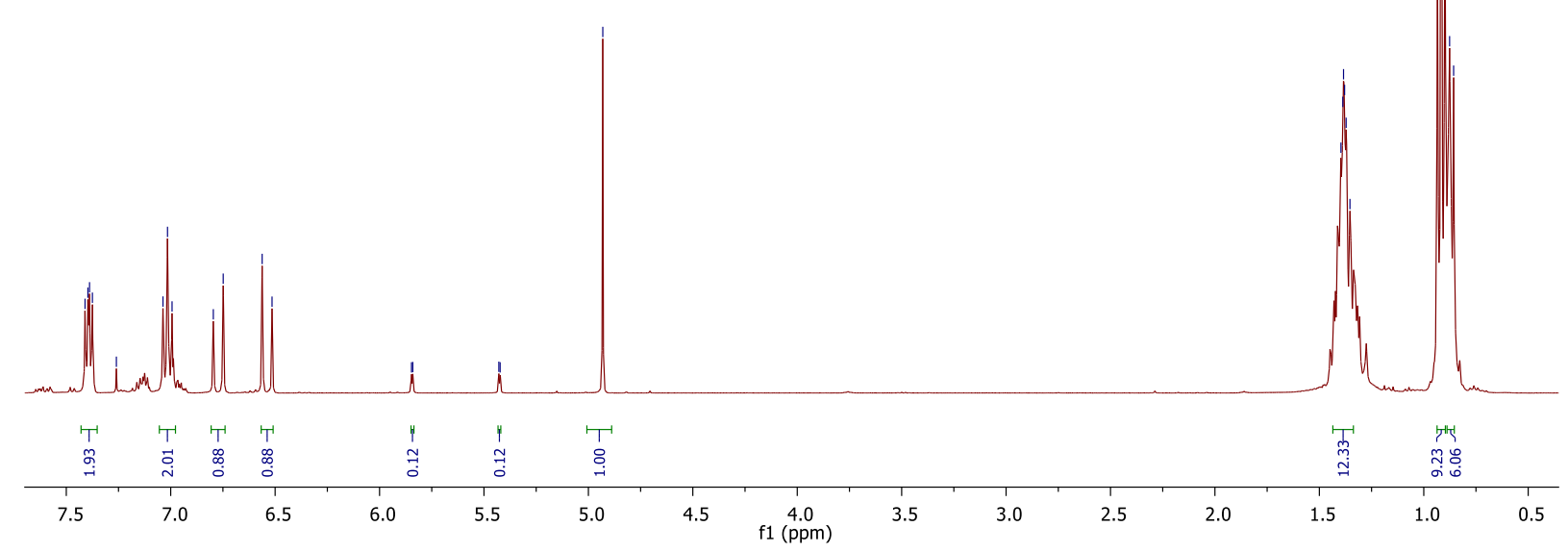

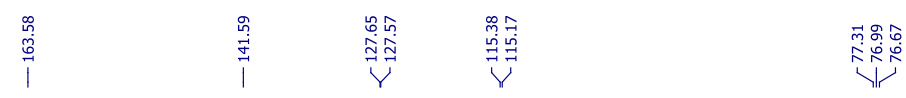

$\mathrm{CDCl}_{3}, 101 \mathrm{MHz}$
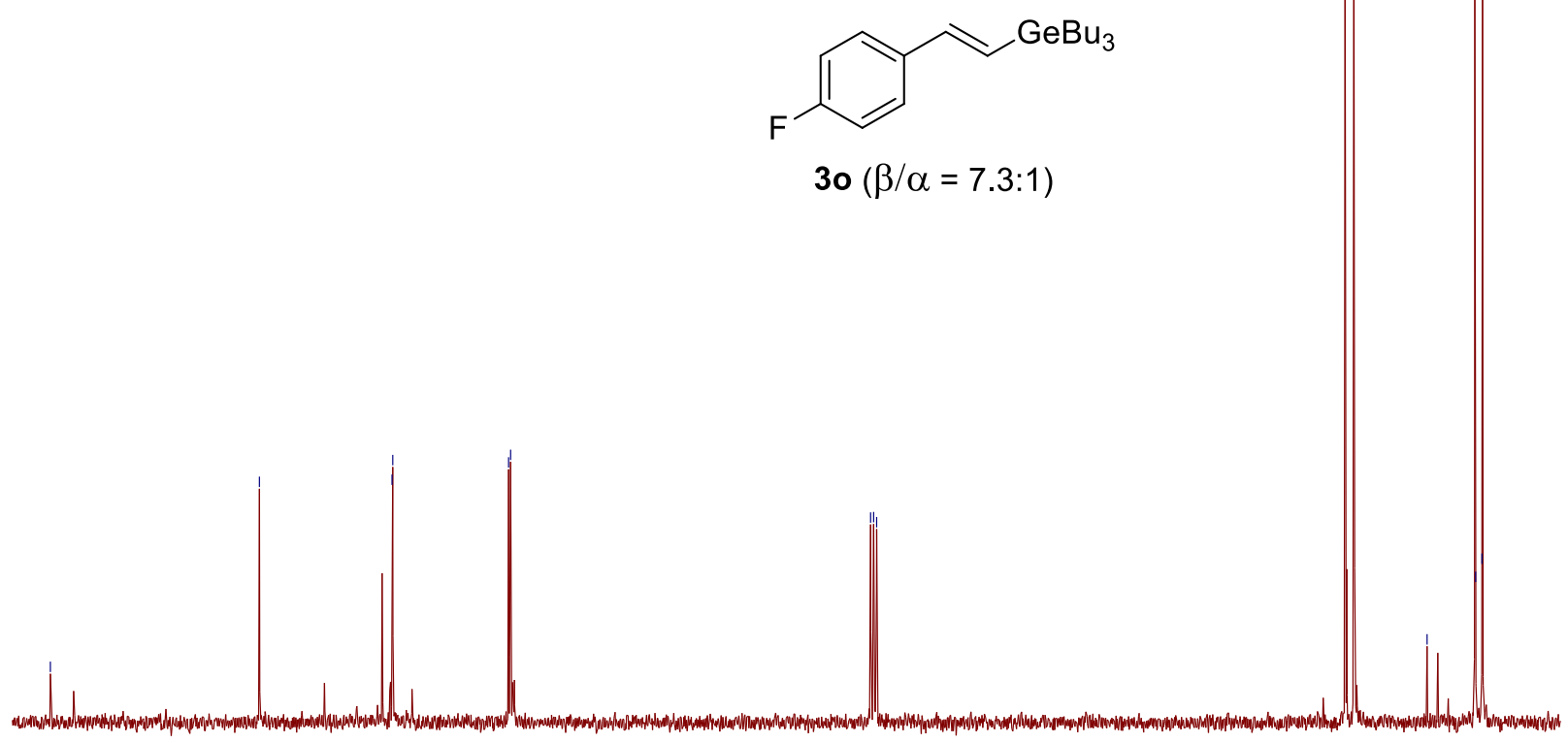

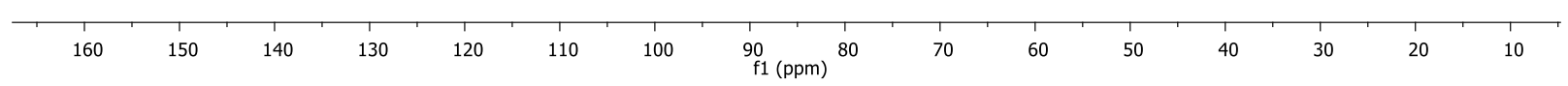




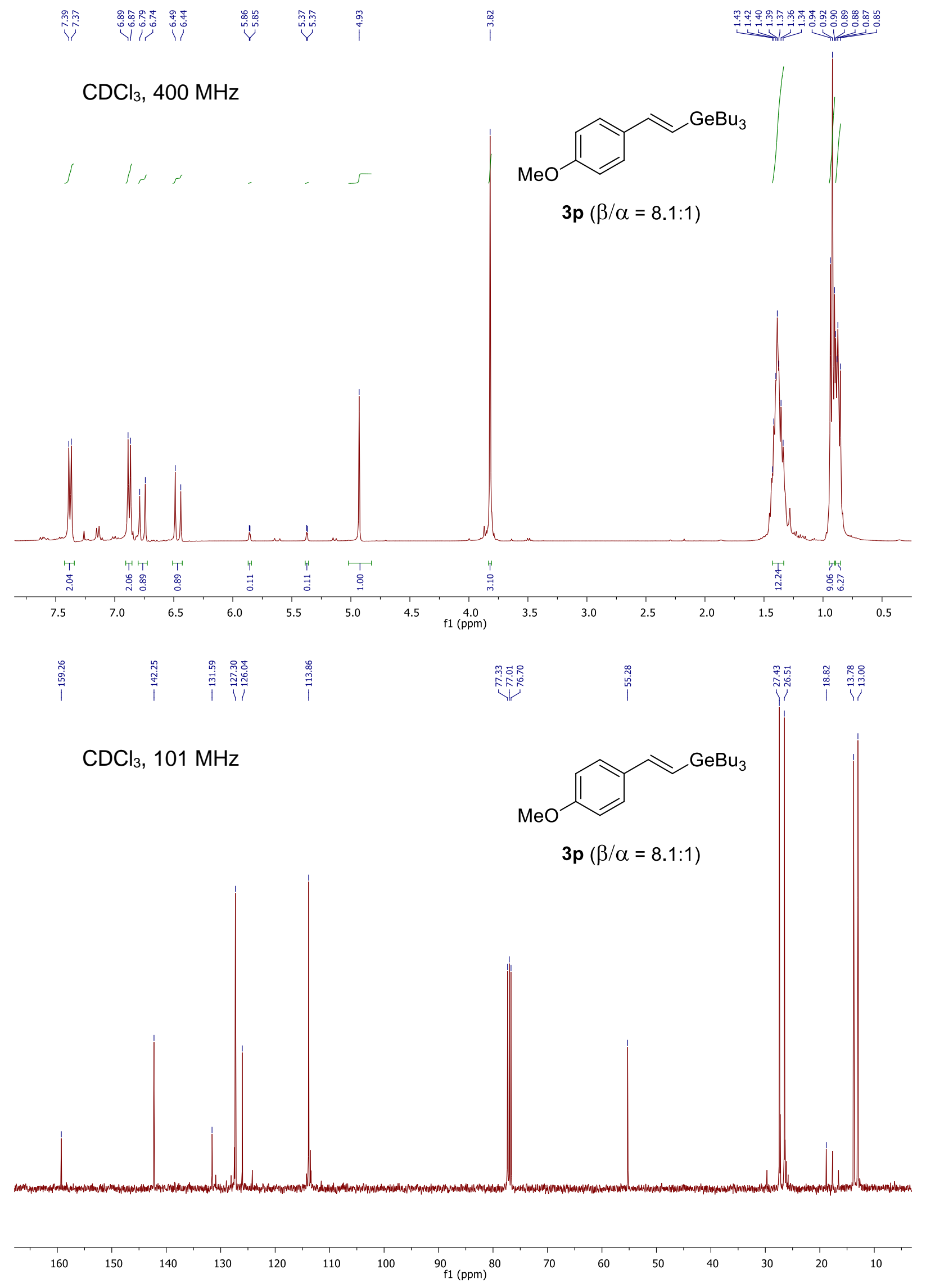




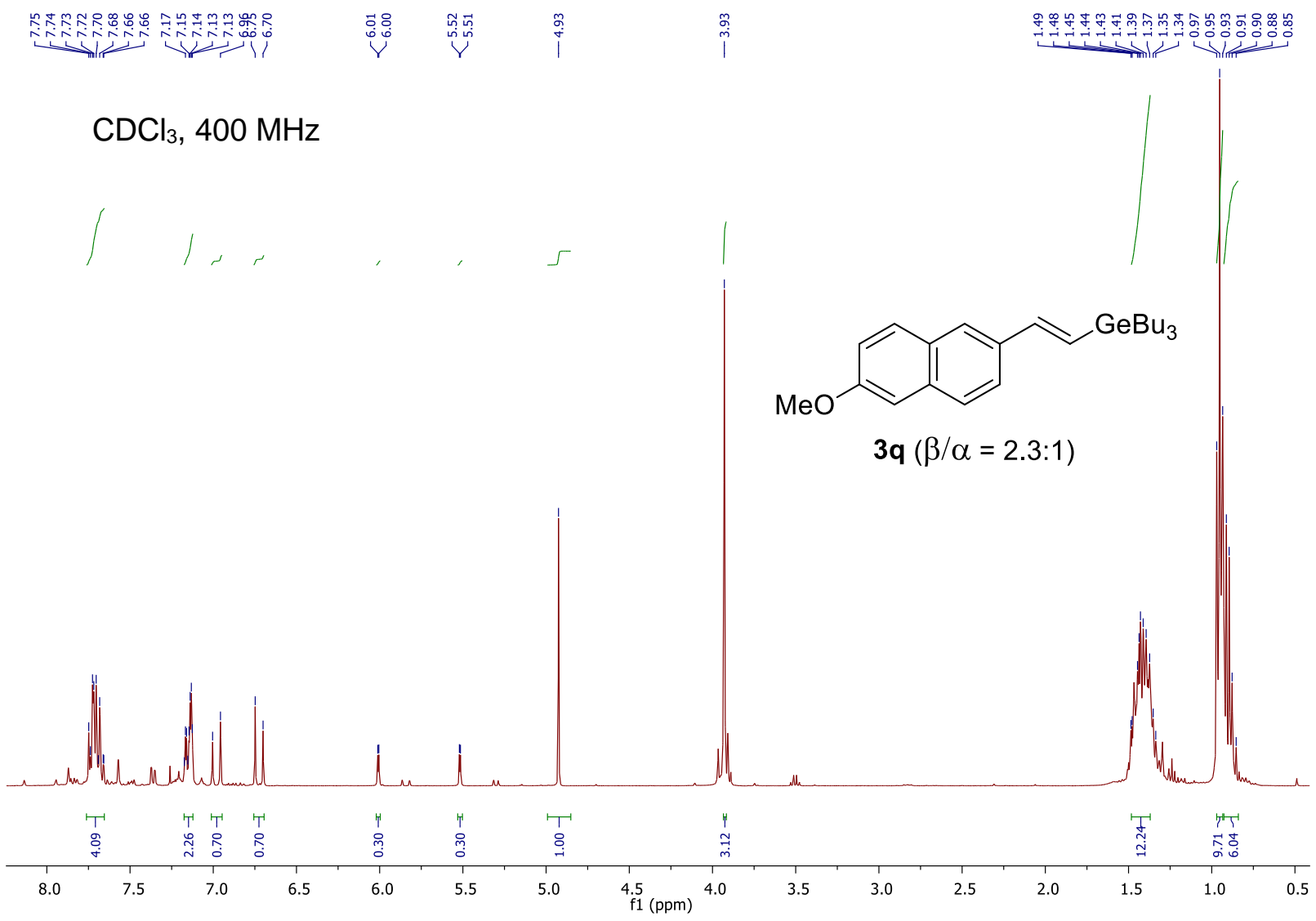

I

$\mathrm{CDCl}_{3}, 101 \mathrm{MHz}$
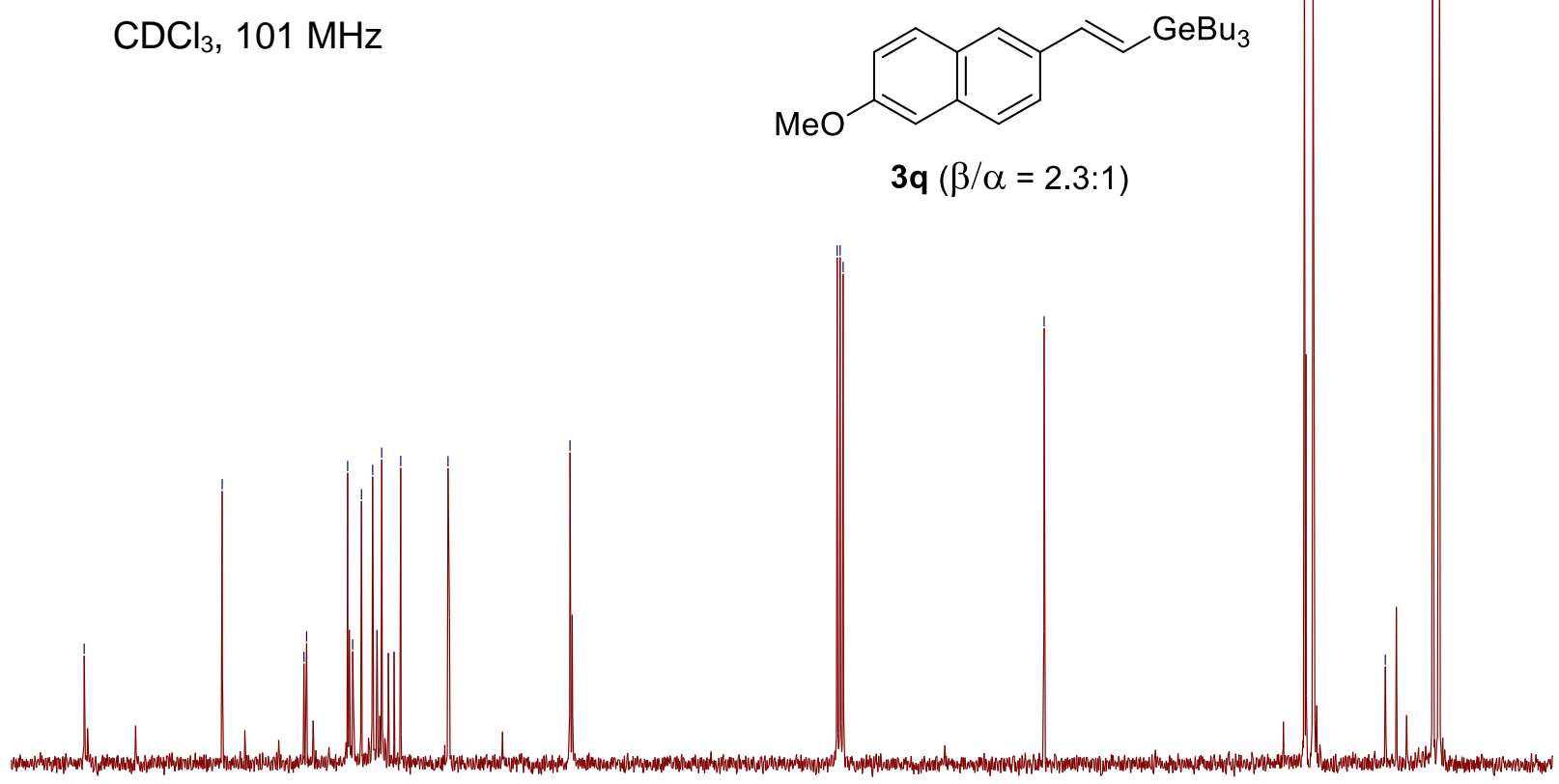

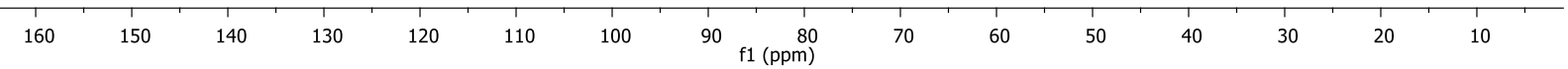




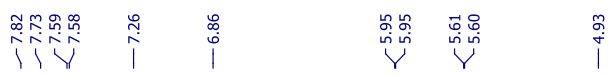

$\mathrm{CDCl}_{3}, 400 \mathrm{MHz}$

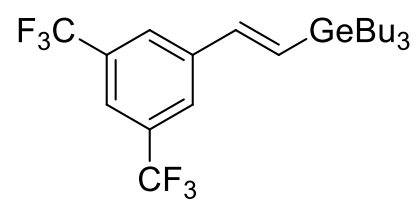

$3 r(\beta / \alpha=3.8: 1)$

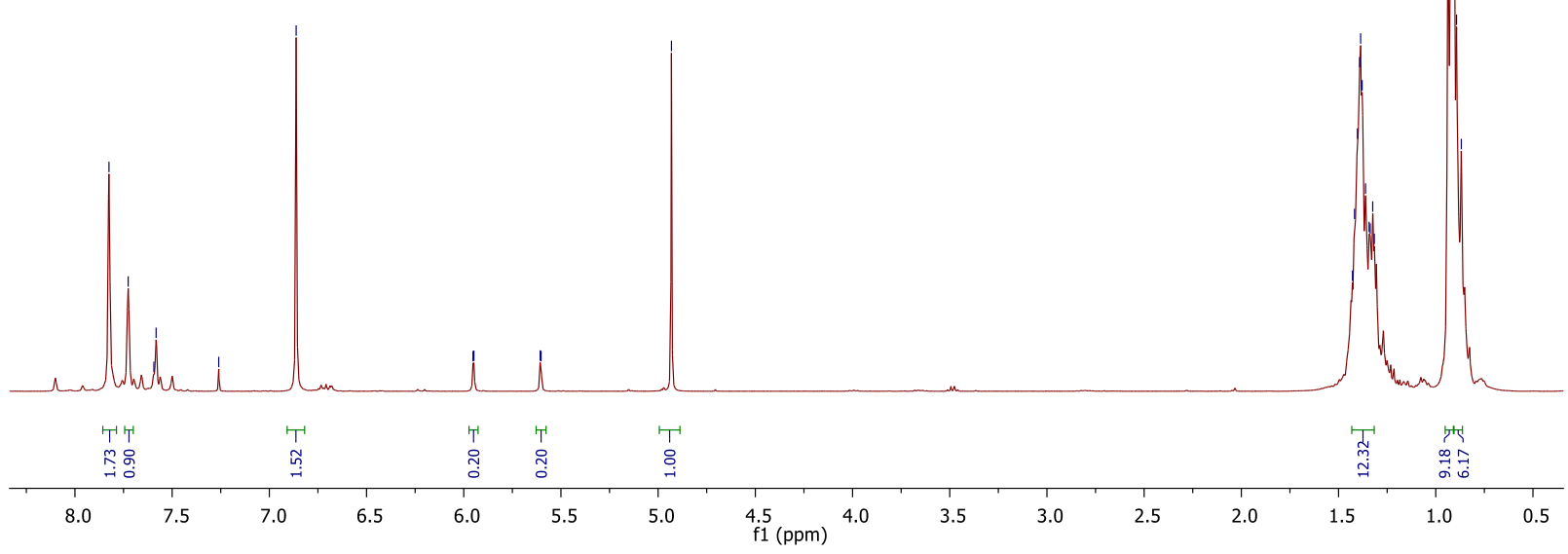

\丶

Acetone-d6, $400 \mathrm{MHz}$
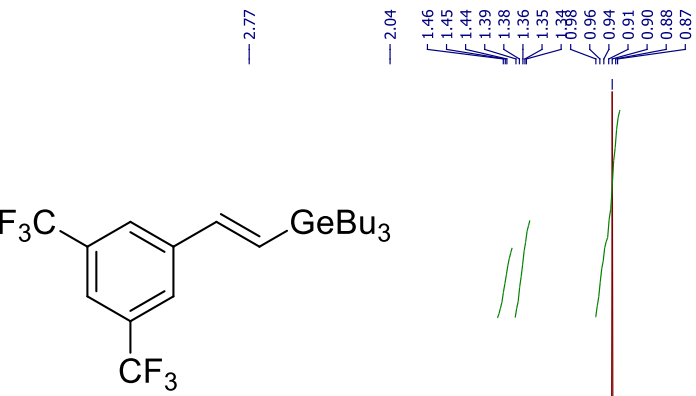

$3 r(\beta / \alpha=3.8: 1)$

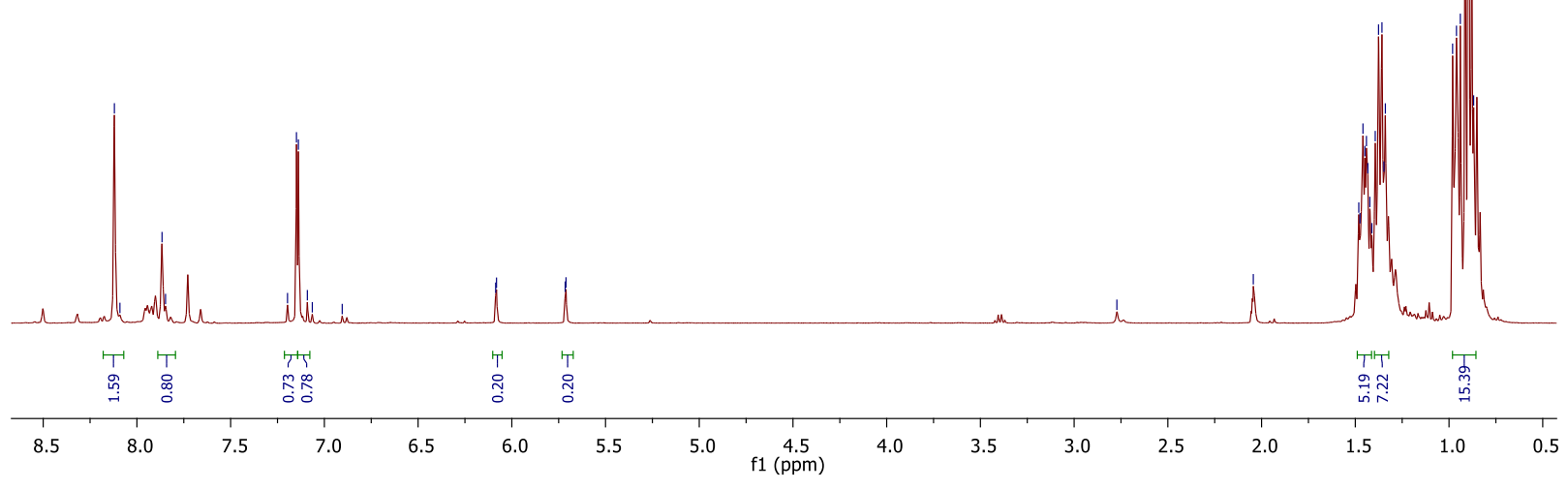




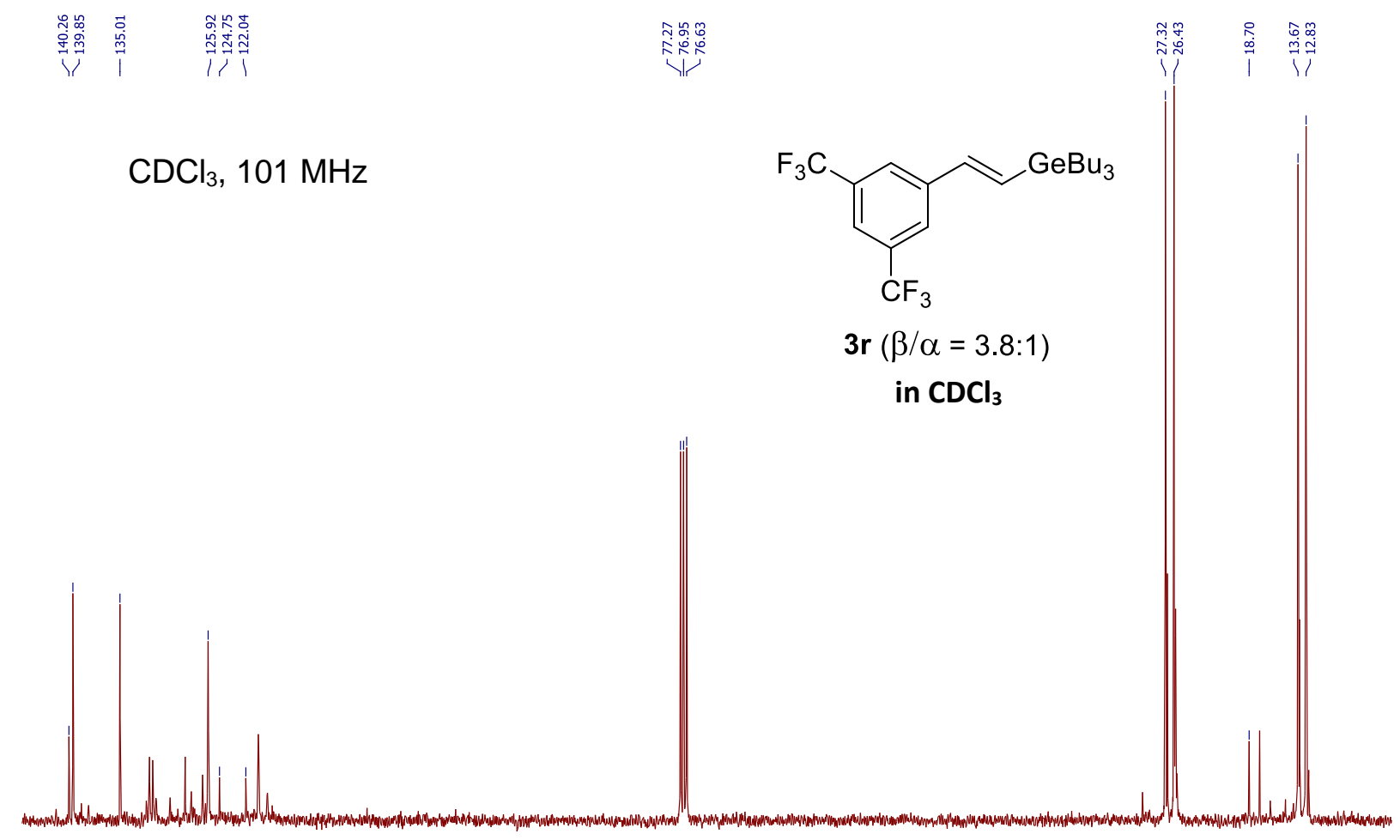

\begin{tabular}{llllllllllllllllllllllllllllllllllllllllllllllllllll}
\hline 145 & 140 & 135 & 130 & 125 & 120 & 115 & 110 & 105 & 100 & 95 & 90 & 85 & 80 & 75 & 70 & 65 & 60 & 55 & 50 & 45 & 40 & 35 & 30 & 25 & 20 & 15 & 10 & 5
\end{tabular}

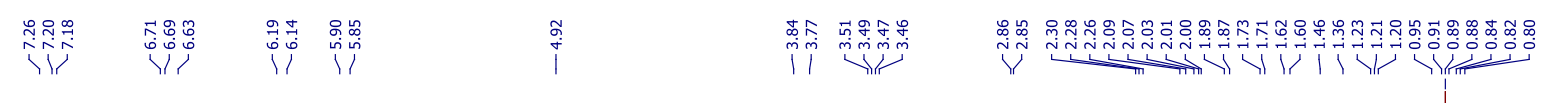

$\mathrm{CDCl}_{3}, 400 \mathrm{MHz}$
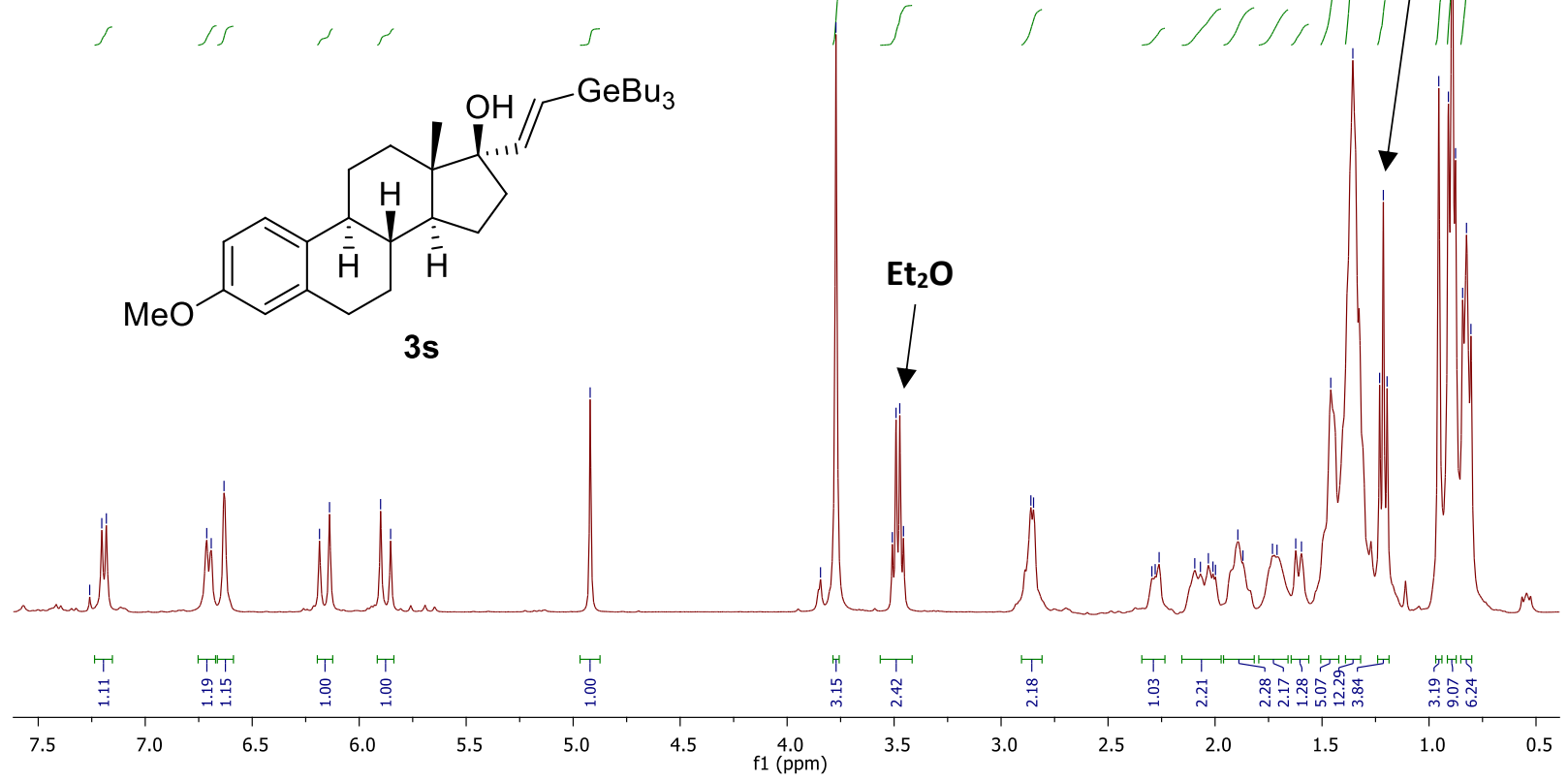


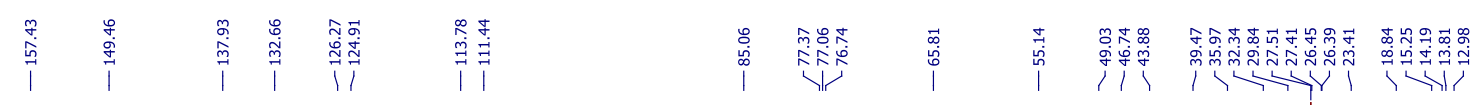

$\mathrm{CDCl}_{3}, 101 \mathrm{MHz}$

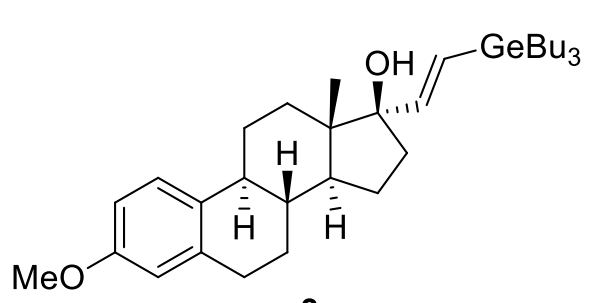

$3 s$

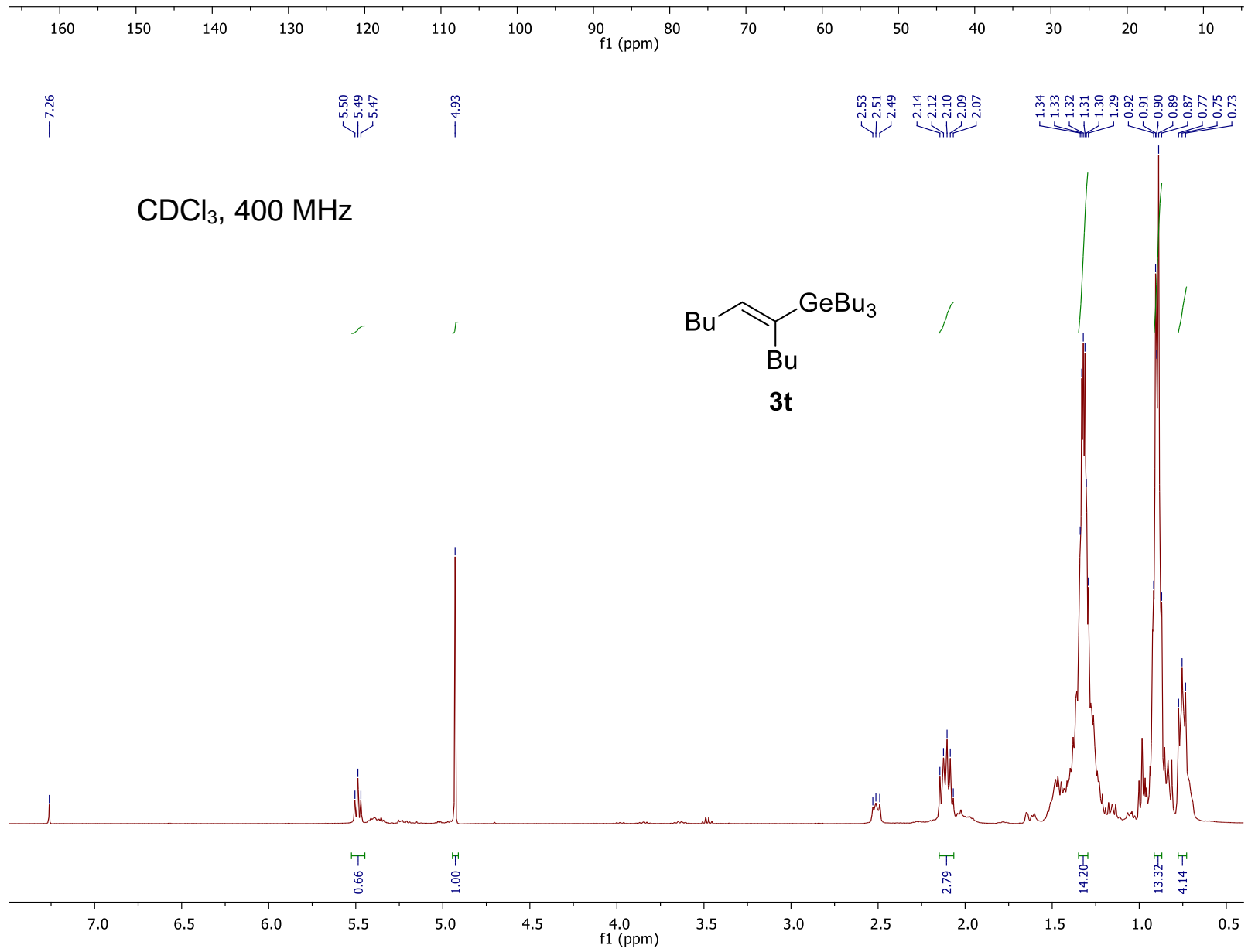



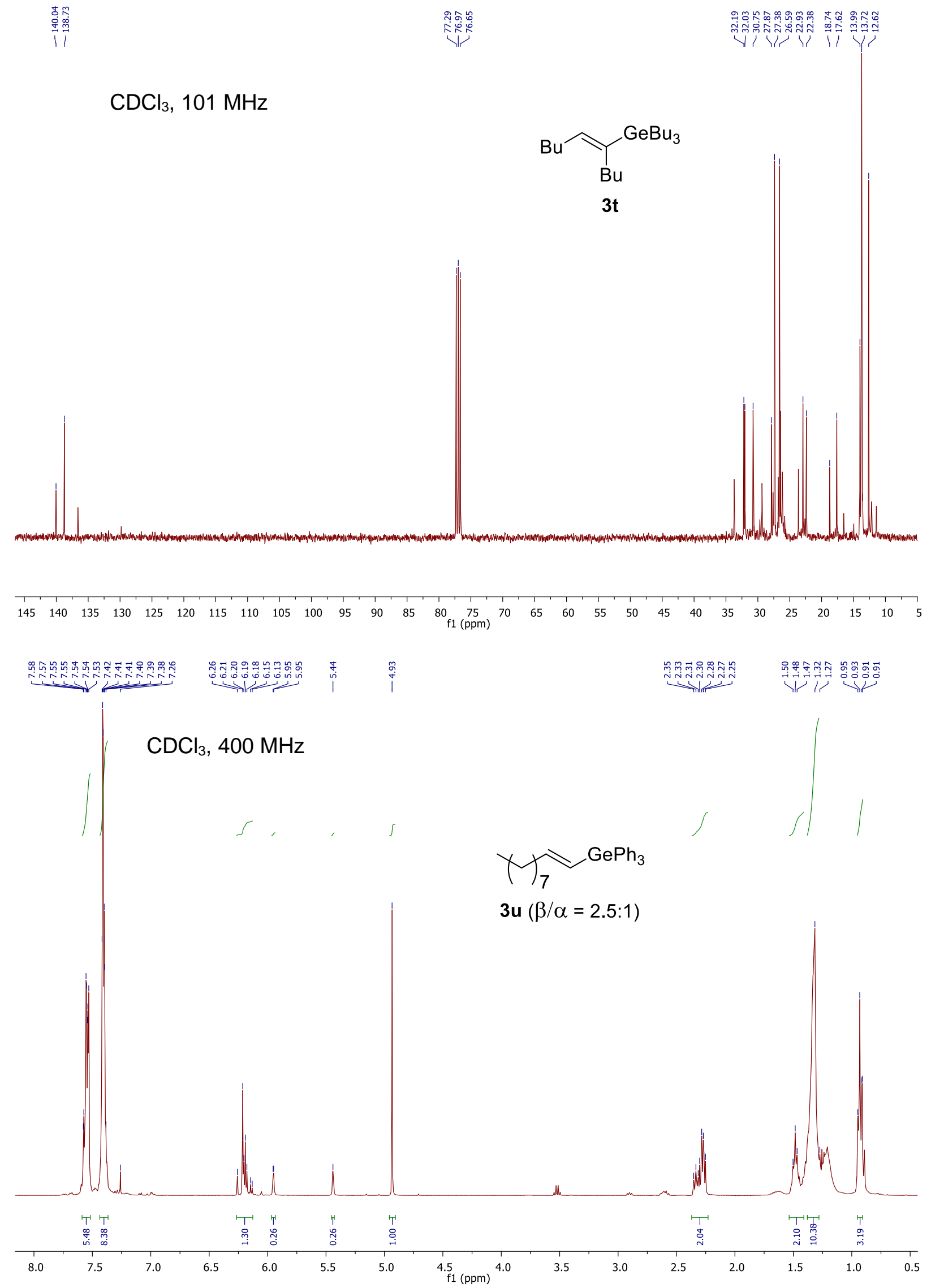

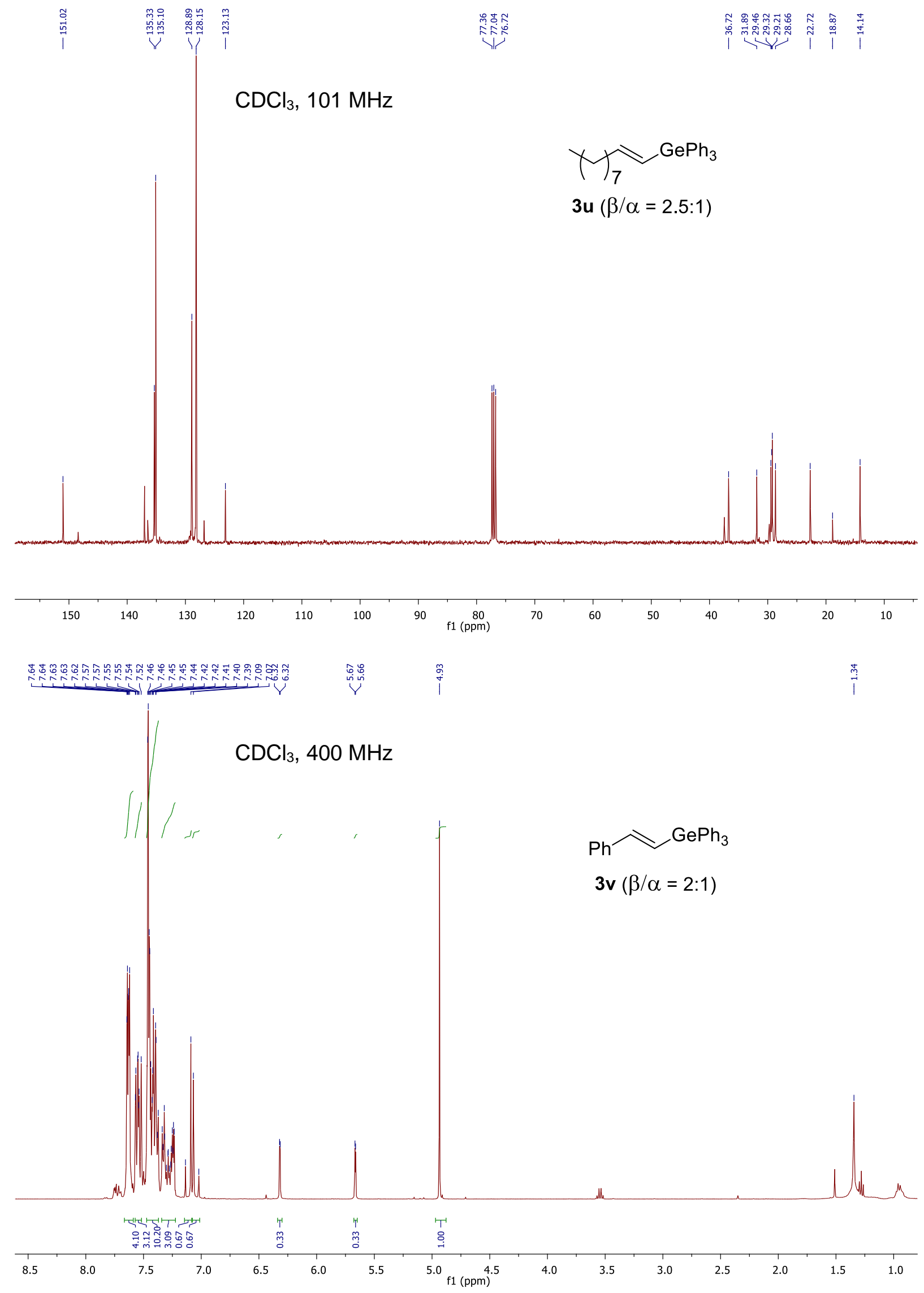

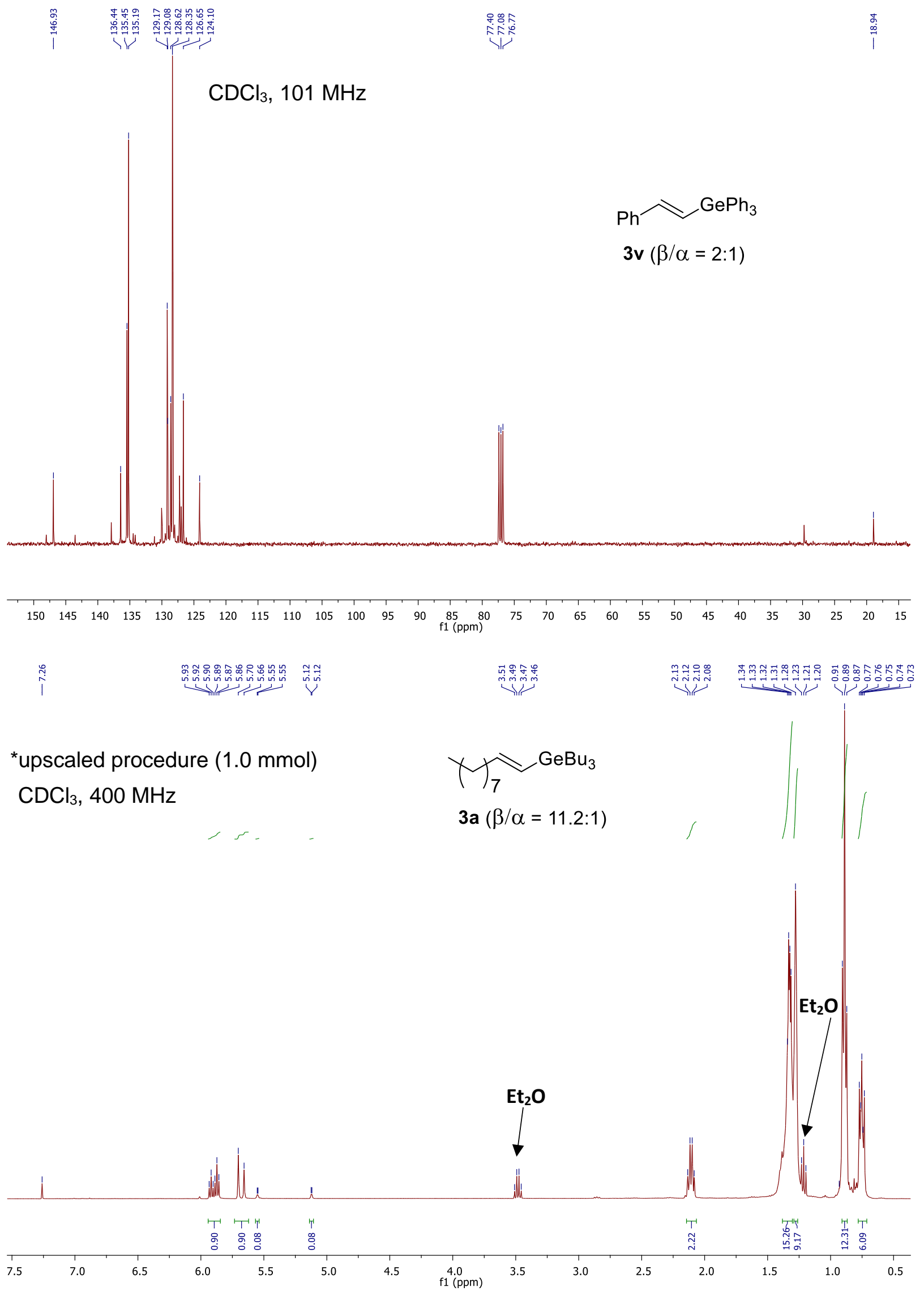


\section{References}

(1) Xie, W.; Chang, S. [Cu(NHC)]-Catalyzed C-H Allylation and Alkenylation of Both Electron-Deficient and Electron-Rich (Hetero)Arenes with Allyl Halides. Angew. Chemie Int. Ed. 2016, 55 (5), 1876-1880.

https://doi.org/10.1002/anie.201510180.

(2) Banerjee, S.; Karunananda, M. K.; Bagherzadeh, S.; Jayarathne, U.; Parmelee, S. R.; Waldhart, G. W.; Mankad, N. P. Synthesis and Characterization of Heterobimetallic Complexes with Direct Cu-M Bonds ( $\mathrm{M}=\mathrm{Cr}$, Mn, Co, Mo, Ru, W) Supported by N-Heterocyclic Carbene Ligands: A Toolkit for Catalytic Reaction Discovery. Inorg. Chem. 2014, 53 (20), 11307-11315. https://doi.org/10.1021/ic5019778.

(3) Behrens, U.; Edelmann, F. Eine Verbesserte Synthese Der Tricarbonyl(Cyclopentadienyl)Metallat-Anionen Des Chroms, Molybdäns Und Wolframs. J. Organomet. Chem. 1984, 263 (2), 179-182. https://doi.org/10.1016/S0022-328X(00)99181-2.

(4) Ohishi, T.; Shiotani, Y.; Yamashita, M. A Convenient One-Flask Preparation of Pure Potassium Cyclopentadienyldicarbonylferrate, K[H5-C5H5) $\mathrm{Fe}(\mathrm{CO}) 2]$. J. Org. Chem. 1994, 59 (1), 250. https://doi.org/10.1021/jo00080a044.

(5) lqbal, M.; Black, R. J. G.; Winn, J.; Reeder, A. T.; Blake, A. J.; Clarke, P. A. Studies on Transannulation Reactions across a Nine-Membered Ring: The Synthesis of Natural Product-like Structures. Org. Biomol. Chem. 2011, 9 (14), 5062-5078. https://doi.org/10.1039/c1ob05448a.

(6) Hou, S.; Yang, H.; Cheng, B.; Zhai, H.; Li, Y. Cobaloxime-Catalyzed Hydration of Terminal Alkynes without Acidic Promoters. Chem. Commun. 2017, 53 (51), 6926-6929. https://doi.org/10.1039/c7cc03919k. 